\title{
Real Hyperbolic on the Outside, Complex Hyperbolic on the Inside
}

\author{
Richard Evan Schwartz *
}

September 19, 2002

\section{Introduction}

The rank one symmetric spaces of negative curvature come in three infinite families: real hyperbolic space $\boldsymbol{H}^{n}$; complex hyperbolic space $\boldsymbol{C H}^{n}$; and quaternionic hyperbolic space $\boldsymbol{Q} \boldsymbol{H}^{n}$. (The Cayley plane is the remaining example.) Aside from the obvious embeddings $\boldsymbol{H}^{n} \hookrightarrow \boldsymbol{C} \boldsymbol{H}^{n} \hookrightarrow \boldsymbol{Q} \boldsymbol{H}^{n}$ the three geometries seem fairly unrelated to each other. For instance, $\boldsymbol{H}^{n}$ admits non-arithmetic lattices in all dimensions $[\mathbf{G r P}]$ while $\boldsymbol{Q} \boldsymbol{H}^{n}$ only admits arithmetic lattices $[\mathbf{G r S}]$. (See $[\mathbf{C}]$ for a related result.) The question of non-arithmetic lattices in $\boldsymbol{C H}^{n}$ is a basic unsolved problem [DM]. For a representation-theoretic comparison of discrete subgroups in the different rank one spaces, see $[\mathbf{S h}]$.

In this paper we make a new connection between $\boldsymbol{H}^{3}$ and $\boldsymbol{C H}^{2}$. We construct a closed hyperbolic 3-manifold which (as a diffeomorphic copy) is the ideal boundary of a complex hyperbolic 4-manifold.

Up to index 2, the isometry group of $\boldsymbol{C H}^{2}$ is $P U(2,1)$, the group of complex projective automorphisms of the unit ball in $\boldsymbol{C}^{2}$. The ideal boundary of $\boldsymbol{C H}^{2}$ is the unit 3-sphere $S^{3}$. A spherical $C R$ structure on a 3-manifold is a system of coordinate charts into $S^{3}$ whose transition functions are restrictions of elements of $P U(2,1)$. While plenty of closed Seifert fibered manifolds admit spherical CR structures $[\mathbf{K T}]$, our example gives the only known spherical CR structure on a closed hyperbolic 3-manifold.

* Supported by N.S.F. Grant DMS-0072607 


\subsection{Statement of Results}

A complex reflection is an element in $P U(2,1)$ conjugate to the mapping $(z, w) \rightarrow(w, z)$. See $\S 2$ for more details. Let $G(4, n)$ be the abstract group with presentation

$$
G(4, n)=\left\langle i_{1}, i_{2}, i_{3} \mid i_{i}^{2}=\left(i_{i} i_{j}\right)^{4}=\left(i_{i} i_{j} i_{i} i_{k}\right)^{n}=e\right\rangle
$$

(All possible pairwise unequal indices are meant to occur.) We will construct, for each $n=5,6,7 \ldots$, a representation $\rho(4, n): G(4, n) \rightarrow P U(2,1)$ such that $I_{j}=\rho\left(i_{j}\right)$ is a complex reflection. Let $\Gamma(4, n)=\rho(4, n)(G(4, n))$. Assuming that $\rho(4, n)$ is discrete, let $\Omega(4, n) \subset S^{3}$ be the domain of discontinuity and let $\Lambda(4, n)=S^{3}-\Omega(4, n)$ be the limit set. Let $A$ and $B$ be the standard generators of the 3 -strand braid group, shown in Figure 5.10.

Theorem 1.1 (Main Theorem) $\rho(4,7)$ is discrete and $\Omega(4,7) / \Gamma(4,7)$ is the hyperbolic orbifold whose underlying space is $S^{3}$ and whose singularity locus is the closed braid $(A B)^{15}\left(A B^{-2}\right)^{3}$, equipped with a $\boldsymbol{Z} / 2$ cone structure.

Passing to a torsion-free finite index subgroup of $\Gamma(4,7)$ we produce the closed hyperbolic 3-manifold which bounds a complex hyperbolic 4-manifold. The orbifold from Theorem 1.1 is double covered by a manifold $M_{7}$ which is an integer slope Dehn-filling of the once-punctured torus bundle with monodromy matrix

$$
\left[\begin{array}{ll}
3 & 1 \\
2 & 1
\end{array}\right]
$$

Our example is a finite sheeted cover of $M_{7}$.

There is some literature on 3-orbifolds whose singularity locus is a particular link in $S^{3}$. See, for instance, $[\mathbf{M V}]$ and $[\mathbf{M W}]$. As far as we know, this literature does not cover our specific example.

It seems that Theorem 1.1 is true for all $n \geq 7$. In general, the braid seems to be $(A B)^{15}\left(A B^{5-n}\right)^{3}$. The group $\Gamma(4,6)$ has a cusp and the group $\Gamma(4,5)$ does not fit the general pattern.

$\Lambda(4, n)$ is a nice limit set. An $\boldsymbol{R}$-circle is the ideal boundary of a totally real, totally geodesic plane in $\boldsymbol{C H}^{2}$. These curves are simple analytic loops in $S^{3}$, integral to the standard contact structure. See $\S 2$ for details.

Theorem $1.2 \Lambda(4, n)$ is the closure of a countable, connected, invariant, nonplanar union of $\boldsymbol{R}$-circles, each of which is stabilized by a subgroup of $\Gamma(4, n)$ conjugate to the standard $(2,4, n)$-reflection triangle group. 
We think that $\Lambda(4, n)$ is homeomorphic to the Menger curve. (See the remark at the end of §3.3.) In light of $[\mathbf{K K}]$ such a result would not be too surprising. Note that Theorem 1.2 only has content when we know $\Omega(4, n)$ is nonempty, as we do in the case $n=7$.

Theorem 1.3 Let $\tau$ be the trace of an element in $\Gamma(4, n)$. Then $2 \Re(\tau)$ and $|\tau|^{2}$ both belong to the ring $\boldsymbol{Z}[2 \cos (2 \pi / n)]$.

All nontrivial Galois conjugates of $\cos (2 \pi / n)$ are negative if $n=5,6,7,8,12$. As we will see in $\S 3$, this fact combines with Theorem 1.3 to prove

Corollary $1.4 \rho(4, n)$ is discrete for $n=5,6,7,8,12$.

Theorem 1.1 and Corollary 1.4 give two logically independent proofs that our main object of study, $\rho(4,7)$, is a discrete representation.

\subsection{Some History}

While self-contained, this paper fits into a progression of papers. In [GP] Goldman and Parker introduced the complex hyperbolic deformations of the ideal triangle group-the complex hyperbolic ideal triangle groups-and partially classified them with respect to discreteness.

In [S1] we proved the Goldman-Parker Conjecture, which settles the discreteness question for these groups. It turns out that the moduli space of discrete complex hyperbolic ideal triangle groups is an interval. All the representations in this interval are also faithful.

In [S2] we analyzed the complex hyperbolic ideal triangle groups which lie at the endpoints of the discreteness interval. (The two groups are conjugate.) We showed that the corresponding orbifold at infinity is commensurable to the Whitehead link complement, a familiar manifold which admits a complete hyperbolic metric of finite volume.

In his thesis [W-G], Justin Wyss-Gallifent discovered a great surprise in connection with deformations of the $(4,4, \infty)$-reflection triangle group. In addition to the expected interval of discrete faithful representations, there seems to be an extra countable collection of representations which are discrete but not faithful.

Motivated by $[\mathbf{S 2}]$ and $[\mathbf{W}-\mathbf{G}]$, I guessed that there should exist extra deformations for the $(4,4,4)$-reflection triangle group, and that these deformations should have closed hyperbolic orbifold quotients at infinity. This paper works out the example which seemed the most amenable to analysis. 
Java Applet 29 on my website [S3] lets the user explore the complex hyperbolic deformations of the hyperbolic $(p, q, r)$ triangle groups. Operating this program, I can see that the extra deformations always exist when $\min (p, q, r) \leq 9$ and never exist when $\min (p, q, r) \geq 14$. The situation is rather complicated between these two ranges. I would love to understand these examples systematically.

Given the vast number of closed hyperbolic 3-manifolds, and the vast number of discrete groups acting on the complex hyperbolic plane, I think that the example in this paper must be common-though perhaps all examples are fairly intricate. We certainly have no general theory. The subject of complex hyperbolic Kleinian groups is still a young subject. (For other papers on complex hyperbolic Kleinian groups, see $[\mathbf{F Z}],[\mathbf{G K L}],[\mathbf{G u P}],[\mathbf{S 1}]$, $[\mathbf{S 2}]$, [Tol] and $[\mathbf{W}-\mathbf{G}]$, as well as the the bibliography in $[\mathbf{G}]$.)

\subsection{Overview of the Paper}

In $\S 2$ we will give some standard background material.

In $\S 3$ we give straightforward proofs of Theorem 1.2, Theorem 1.3, and Corollary 1.4. The rest of the paper is devoted to proving Theorem 1.1.

The proof of Theorem 1.1 centers around the construction of a simplicial complex $Z \subset C^{2,1}$. The vertices of $Z$ are canonical lifts to $C^{2,1}$ of fixed points of certain elements of $\Gamma(4,7)$. The tetrahedra of $Z$ are Euclidean convex hulls of various 4 -element subsets of the vertices. Comprised of infinitely many tetrahedra, $Z$ is invariant under an element $K$ which has the property that $K^{3}=-I_{2} I_{1} I_{3}$. Modulo $K$, our complex $Z$ has finitely many tetrahedra.

Let [ ] : $\boldsymbol{C}^{2,1}-\{0\} \rightarrow \boldsymbol{C} \boldsymbol{P}^{2}$ be the projectivization map. (See Equation 5 for a formula.) Let $\left[Z_{0}\right]=[Z] \cap S^{3}$. Let $\left[E_{0} K\right]$ and $\left[E_{\infty} K\right]$ be the two fixed points, in $S^{3}$, of $K$. (These points are the projectivizations of the null eigenvectors $E_{0} K$ and $E_{\infty} K$ of $K$.) The proof of Theorem 1.1 relies on two facts which we call the tiling hypotheses.

1. $\left[Z_{0}\right] \cup\left[E_{0} K\right] \cup\left[E_{\infty} K\right]$ is an embedded 2-sphere.

2. One of the two components $\Delta_{0}$ of $S^{3}-\left(\left[Z_{0}\right] \cup\left[E_{0} K\right] \cup\left[E_{\infty} K\right]\right)$ is such that $\Omega(4,7)$ is tiled by the $\Gamma(4,7)$-orbit of $\Delta_{0} \cup\left[Z_{0}\right]$.

In $§ 4-5$ we establish Theorem 1.1 assuming the tiling hypotheses. The simplicial structure of $Z$ gives rise to a tiling of $\left[Z_{0}\right]$. In $\S 4$ we define $Z$ and work out the combinatorics of the tiling on $\left[Z_{0}\right]$. Figures 4.1 and 4.9 show 
pictures of this tiling drawn in coordinate systems which are adapted to the CR geometry of $S^{3}$. The various tiles of $\left[Z_{0}\right]$ are paired together by certain elements in $\Gamma(4,7)$.

In $\S 5$ we construct a lattice $\Gamma^{*}(4,7) \subset P S L_{2}(\boldsymbol{C})$ and a surjective homomorphism $h: \Gamma^{*}(4,7) \rightarrow \Gamma(4,7)$. We use this lattice to build an infinite polyhedron $Z_{0}^{*} \subset \boldsymbol{H}^{3}$ that serves as a kind of hyperbolic transcription of $\left[Z_{0}\right]$. The various tiles of $Z_{0}^{*}$ are paired together by certain elements in $\Gamma^{*}(4,7)$ and one of the components $\Delta_{0}^{*}$ of $\boldsymbol{H}^{3}-Z_{0}^{*}$ plays the role of $\Delta_{0}$. Compare the plot of $Z_{0}^{*}$ shown in Figure 5.6 with the plot of $\left[Z_{0}\right]$ shown in Figure 4.1. There is a "symmetry respecting" homeomorphism $h^{\prime}:\left(\Delta_{0}^{*}, Z_{0}^{*}\right) \rightarrow\left(\Delta_{0},\left[Z_{0}\right]\right)$ and a universal covering map $h^{\prime \prime}: \boldsymbol{H}^{3} \rightarrow \Omega(4,7)$. The three maps $h, h^{\prime}, h^{\prime \prime}$ are all compatible, and we use them to put the hyperbolic structure on $\Omega(4,7) / \Gamma(4,7)$. At the end of $\S 5$ we analyze the topology of $\Omega(4,7) / \Delta(4,7)$.

Here is an alternate approach to much of $\S 5$. Once we analyze the topology of $\Omega(4,7) / \Delta(4,7)$, it might be possible to show that the orbifold is hyperbolic by combining results in $[\mathbf{F H}]$ with Thurston's Orbifold Theorem $[\mathbf{B L P}],[\mathbf{C H K}]$. We do not attempt this.

In $\S 6-10$ we establish the tiling hypotheses. In $\S 6$ we develop a combinatorial picture of $[Z]$. We will build $[Z]$ in 3 concentric layers. The outer layer of $[Z]$ is a thickening of $\left[Z_{0}\right]$. We also analyze how the reflection pairings act on $[Z]$. Our picture of $[Z]$ depends on several assumptions we make, which we list in $\S 6.1$. The most important assumption is that the map $Z \rightarrow[Z]$ is a homeomorphism.

Let $\left[Z_{-}\right]=[Z] \cap \boldsymbol{C} \boldsymbol{H}^{2}$. In $\S 7$ we will use the material in $\S 6$ to show that there is a component $\Delta_{-}$of $\boldsymbol{C H}^{2}-\left[Z_{-}\right]$such that the $\Gamma(4,7)$ orbit of $\Delta_{-} \cup\left[Z_{-}\right]$tiles $\boldsymbol{C} \boldsymbol{H}^{2}$. Once we know this, the tiling hypotheses follow readily from standard material on ends of Kleinian groups. We emphasize that our results in $\S 6-7$ depend on the assumptions listed in $\S 6.1$.

In $\S 8$ we will see that, unfortunately, the map $Z \rightarrow[Z]$ is not a homeomorphism. To fix the problem we create $Z^{\prime}$, a sensible but non-canonical replacement for $Z$, by adding extra vertices to $Z$ and perturbing slightly. The perturbation takes place entirely within the set of negative vectors in $C^{2,1}$ so that $\left[Z_{0}\right]=\left[Z_{0}^{\prime}\right]$. All the arguments for $Z$ go through for $Z^{\prime}$ with only trivial modifications.

To complete the proof of Theorem 1.1 we need to verify that $Z^{\prime}$ satisfies the hypotheses listed in $\S 6.1$. Our main task is showing that the projectivization map [ ] is injective on all pairs of tetrahedra within a large but finite portion of $Z^{\prime}$. Roughly, we need to check about 1.3 million tetrahedra. The 
sheer number of checks forces us to bring in the computer.

In $\S 9$ we develop a technique for proving, with rigorous machine-aided computation, that [ ] is injective on a given pair of tetrahedra. To deal with potential roundoff error in the computations we implement interval arithmetic, as we did in [S1].

We discuss the implementation of our code in $\S 10$. The successful running of our code, which takes about 12 hours on a Sparc Ultra 10, is a vital component of our proof.

For the convenience of the reader we include a list of symbols in $\S 11$.

We wrote the small amount computer code pertaining to $\S 5$ in Mathematica $[\mathbf{W}]$. Otherwise, we wrote the code in $\mathrm{C}[\mathbf{K R}]$. We also wrote an extensive graphical user interface in $\mathrm{Tcl} / \mathrm{Tk}[\mathbf{O}]$ which checks that the com-

putations operate as intended. We have tested the code extensively. As a further sanity check, have used the code, in conjunction with the graphical user interface, to generate the many computer plots in the paper. One can download all the computer code from our website [S3].

Our proof of Theorem 1.1 uses plenty of complex affine geometry, but essentially no complex hyperbolic geometry. The reader may wonder if there is a more intrinsically hyperbolic approach to proving Theorem 1.1. Could we construct an object like $[Z]$ using patently complex hyperbolic objects? We think that such an approach should be possible, but we simply do not know how to verify that it works. For instance, it seems to us that a computeraided proof would require far too much computation to be practical.

\subsection{Acknowledgements}

I would like to thank Martin Bridgeman, Brienne Brown, Peter Doyle, Nathan Dunfield, Bill Goldman, Jeremy Kahn, Misha Kapovich, Bruce Kleiner, Greg Liebon, John Millson, John Parker, Bill Thurston, Max Wardetsky, and Justin Wyss-Gallifent for helpful and interesting conversations related to this paper. I would also like to thank the referee for many helpful comments. In particular, the slick proof of Lemma 5.1 is mainly due to the referee.

I dedicate this paper to my daughter, Lilith Antoinette Schwartz, born June 30, 2000. 


\section{Background Information}

We try to keep our conventions consistent with $[\mathbf{G}]$, which is our main reference for the material in this chapter. $[\mathbf{E}]$ is another excellent reference.

Complex Hermitian Space: $\boldsymbol{C}^{n, 1}$ is a copy of the vector space $\boldsymbol{C}^{n+1}$ equipped with the Hermitian form

$$
\langle U, V\rangle=-u_{n+1} \bar{v}_{n+1}+\sum_{j=1}^{n} u_{j} \bar{v}_{j}
$$

Here $U=\left(u_{1}, \ldots, u_{n+1}\right)$ and $V=\left(v_{1}, \ldots, v_{n+1}\right)$. Vectors in the sets

$$
\begin{aligned}
& N_{-}=\left\{V \in \boldsymbol{C}^{n, 1} \mid\langle V, V\rangle<0\right\} ; \\
& N_{0}=\left\{V \in \boldsymbol{C}^{n, 1} \mid\langle V, V\rangle=0\right\} ; \\
& N_{+}=\left\{V \in \boldsymbol{C}^{n, 1} \mid\langle V, V\rangle>0\right\}
\end{aligned}
$$

are respectively called negative, null, and positive.

Complex Hyperbolic Space: $\boldsymbol{C}^{n}$ includes in complex projective space $\boldsymbol{C} \boldsymbol{P}^{n}$ as the set of vectors with nonzero last coordinate. We call this copy of $\boldsymbol{C}^{n}$ the affine patch. Let []: $\boldsymbol{C}^{n, 1}-\{0\} \rightarrow \boldsymbol{C} \boldsymbol{P}^{n}$ be the projectivization whose formula, expressed in the affine patch, is

$$
\left[\left(v_{1}, \ldots, v_{n+1}\right)\right]=\left(v_{1} / v_{n+1}, \ldots, v_{n} / v_{n+1}\right)
$$

Complex hyperbolic space, $\boldsymbol{C H} \boldsymbol{H}^{n}$, is the projective image of the set of negative vectors in $\boldsymbol{C}^{n, 1}$. That is, $\boldsymbol{C} \boldsymbol{H}^{n}=\left[N_{-}\right]$. The ideal boundary of $\boldsymbol{C H}^{n}$ is the unit sphere $S^{2 n-1}=\left[N_{0}\right]$. If $[X],[Y] \in \boldsymbol{C H}^{n}$ the complex hyperbolic distance $\varrho([X],[Y])$ satisfies

$$
\varrho([X],[Y])=2 \cosh ^{-1} \sqrt{\delta(X, Y)} ; \quad \delta(X, Y)=\frac{\langle X, Y\rangle\langle Y, X\rangle}{\langle X, X\rangle\langle Y, Y\rangle} .
$$

Here $X$ and $Y$ are arbitrary lifts of $[X]$ and $[Y]$. See $[\mathbf{G}$, p. 77]. Until $\S 9$ we will take $n=2$. Thus the model for $\boldsymbol{C H}^{2}$ is the open unit ball in $\boldsymbol{C}^{2}$ and the ideal boundary is $S^{3}$. 
Isometries: $S U(2,1)$ is the group of $\langle$,$\rangle preserving complex linear transfor-$ mations. $P U(2,1)$ is the projectivization of $S U(2,1)$ and acts isometrically on $\boldsymbol{C H}^{2}$. Explicitly, given $T \in S U(2,1)$ and $v=\left(v_{1}, v_{2}\right) \in \boldsymbol{C H}^{2}$ we define $T \in P U(2,1)$ by the action

$$
T(v)=\left[T\left(v_{1}, v_{2}, 1\right)\right] .
$$

The map $S U(2,1) \rightarrow P U(2,1)$ is a 3 -to-1 Lie group homomorphism. The group of holomorphic isometries of $\boldsymbol{C H}^{2}$ is exactly $P U(2,1)$. The full group of isometries of $\boldsymbol{C} \boldsymbol{H}^{2}$ is generated by $P U(2,1)$ and by the antiholomorphic $\operatorname{map}\left(z_{1}, z_{2}, z_{3}\right) \rightarrow\left(\bar{z}_{1}, \bar{z}_{2}, \bar{z}_{3}\right)$.

An element of $P U(2,1)$ is called elliptic if it has a fixed point in $\boldsymbol{C H}^{2}$. It is called hyperbolic (or loxodromic) if there is some $\epsilon>0$ such that every point in $\boldsymbol{C H}^{2}$ is moved at least $\epsilon$ by the isometry.. An element which is neither elliptic nor hyperbolic is called parabolic. See $[\mathbf{G}, \S 6.2]$ for more details.

A loxodromic element of $S U(2,1)$ has two null eigenvectors $U$ and $V$, and these correspond to its unique two fixed points $[U],[V] \in S^{3}$. The vector $U \otimes V \in N_{+}$is also an eigenvector. Here

$$
U \otimes V=\left(\overline{u_{3} v_{2}-u_{2} v_{3}}, \overline{u_{1} v_{3}-u_{3} v_{1}}, \overline{u_{1} v_{2}-u_{2} v_{1}}\right) .
$$

This vector is such that $\langle U, U \otimes V\rangle=\langle V, U \otimes V\rangle=0$. See [G, p. 45].

Complex Reflections: Let $C \in N_{+}$. Given any $U \in C^{2,1}$ define

$$
I_{C}(U)=-U+\frac{2\langle U, C\rangle}{\langle C, C\rangle} C
$$

$I_{C}$ is an involution fixing $C$ and $I_{C} \in S U(2,1)$. See [G, p. 70]. Such maps are called complex reflections. The complex reflections generate $S U(2,1)$.

Every complex reflection is conjugate to the following simple example: Setting $C=(-1,1,0)$, we get $I_{C}\left(u_{1}, u_{2}, u_{3}\right)=\left(-u_{2},-u_{1},-u_{3}\right)$. The projective action is just $I_{C}(z, w)=(w, z)$. One of the eigenspaces of $I_{C}$ corresponds to the eigenvector -1 . This space is spanned by $(0,0,1)$ and $(1,1,0)$. We call this the negative eigenspace. The eigenspace corresponding to the eigenvector 1 is spanned by $(-1,1,0)$. We call this the positive eigenspace. Note that all vectors in the positive eigenspace belong to $N_{+}$. 
Totally Geodesic Slices: $\boldsymbol{C} \boldsymbol{H}^{2}$ has two different kinds of totally geodesic subspaces, complex slices and real slices. A complex slice is the intersection of a complex line in $\boldsymbol{C P}^{2}$ with $\boldsymbol{C H}^{2}$. Complex slices are the fixed point sets, in $\boldsymbol{C H}^{2}$, of complex reflections. A real slice in $\boldsymbol{C} \boldsymbol{H}^{2}$ is the fixed point set of an anti-holomorphic isometry of $\boldsymbol{C H}^{2}$. Every real slice is isometric to $\boldsymbol{R}^{2} \cap \boldsymbol{C H}^{2}$. The ideal boundary, on $S^{3}$, of a real slice, is called an $\boldsymbol{R}$-circle. The subgroup of $P U(2,1)$ stabilizing a given real slice is isomorphic to the isometry group of $\boldsymbol{H}^{2}$. See $[\mathbf{G}, \S 4]$ for more details.

Selection Criterion: We shall have many occasions to pick out one vector in $C^{2,1}$ amongst several closely related ones. We will make the selection based on the real parts of the third coordinates of the vectors. Given $X=\left(x_{1}, x_{2}, x_{3}\right)$ and $Y=\left(x_{1}, y_{2}, y_{3}\right)$ we say that $X$ is higher than $Y$ if $\Re\left(x_{3}\right)>\Re\left(y_{3}\right)$. At the same time we say that $Y$ is lower than $X$. Given a finite collection $\left\{X_{1}, \ldots, X_{k}\right\}$ of vectors we can define the highest and the lowest in the obvious way. As one more bit of terminology, we say that $X$ is high if $\Re\left(x_{3}\right)>0$ and low if $\Re\left(x_{3}\right)<0$.

Notational Convention: We adopt the convention that an element of $S U(2,1)$ is given the same symbol as the corresponding element of $P U(2,1)$. Correspondingly, we make the implicit assumption that elements of $S U(2,1)$ act on $\boldsymbol{C P}^{2}$ via their projectivizations. At first glance, this practice seems to introduce some ambiguity, because the natural map $S U(2,1) \rightarrow P U(2,1)$ is 3 -to-1. However, every element in $P U(2,1)$ we actually consider comes with a preferred lift in $S U(2,1)$. 


\section{The Group}

\subsection{Basic Formulas}

Suppose that $r, s, t \in(0,1)$ are variables such that $r^{2} s^{2}+t^{2}<1$. We define

$$
V_{1}=\kappa_{1}(r s \sqrt{-1}, t, 1) ; \quad V_{2}=\kappa_{2}(-r, 0,1) ; \quad V_{3}=\overline{\kappa_{2}}(r, 0,1) .
$$

The constants $\kappa_{1}$ and $\kappa_{2}$ do not play a role in this section. We will specify them in the next section.

Using Equation 8 as a guide we define

$$
C_{1}=(0,1,0) ; \quad C_{2}=(t, r+r s \sqrt{-1}, r t) ; \quad C_{3}=(-t, r-r s \sqrt{-1}, r t),
$$

so that $\left\langle C_{i}, V_{j}\right\rangle=0$ if $i \neq j$. We set $I_{j}=I_{C_{j}}$, as in Equation 9 .

Let tr denote trace. To make $I_{i} I_{j}$ be a rotation by $\pi / 2$ we set $\operatorname{tr}\left(I_{i} I_{j}\right)=1$. Alternatively, we require that

$$
\delta\left(C_{i}, C_{j}\right)=1 / 2
$$

Here $\delta$ is as in Equation 6. This last equation forces the complex lines fixed by $I_{i}$ and $I_{j}$ to meet at an angle of $\pi / 4$. See [G, p. 100]. Solving for $s$ and $t$ in terms of $r$ we get:

$$
s^{2}=\frac{1-2 r^{2}-r^{4}}{1+2 r^{2}-r^{4}} ; \quad t^{2}=\frac{2\left(r^{2}+r^{4}\right)}{1+2 r^{2}-r^{4}} .
$$

To make $I_{1} I_{2} I_{1} I_{3}$ a rotation by $2 \pi / n$ we set $\operatorname{tr}\left(I_{1} I_{2} I_{1} I_{3}\right)=1+2 \cos (2 \pi / n)$ and solve for $r$ :

$$
r^{2}=\frac{\cos (\pi / n)-\cos (\pi / 4)}{\cos (\pi / n)+\cos (\pi / 4)}
$$

The other relations $\operatorname{tr}\left(I_{i} I_{j} I_{i} I_{k}\right)=1+2 \cos (2 \pi / n)$ follow from symmetry.

Fixing $n \in\{5,6,7 \ldots\}$ we let $G(4, n)$ be the group in Equation 1. We define $\rho=\rho(4, n)$ by the equation $\rho\left(i_{j}\right)=I_{j}$, where $I_{j}$ is defined in terms of $r, s, t$, as above. (Any nonzero choices for $\kappa_{1}$ and $\kappa_{2}$ lead to the same complex reflections.) We set $\Gamma(4, n)=\rho(G(4, n))$.

Independent of $\kappa_{1}$ and $\kappa_{2}$ the triangle $\left\{V_{1}, V_{2}, V_{3}\right\}$ satisfies

$$
\delta\left(V_{i}, V_{j}\right)=1+\cos (2 \pi / n) ; \quad \delta\left(V_{k}, I_{k}\left(V_{k}\right)\right)=(1+\cos (2 \pi / n))^{2} .
$$

The action of $\Gamma(4, n)$ on $\boldsymbol{C H}^{2}$ is determined by the equilateral triangle with vertices $\left[V_{1}\right],\left[V_{2}\right],\left[V_{3}\right]$. 


\subsection{Some Auxilliary Elements}

We now explain how to choose $\kappa_{1}$ and $\kappa_{2}$ so that

$$
\left\langle V_{1}, V_{2}\right\rangle=\left\langle V_{2}, V_{3}\right\rangle=\left\langle V_{3}, V_{1}\right\rangle ; \quad\left\langle V_{1}, V_{1}\right\rangle=\left\langle V_{2}, V_{2}\right\rangle=\left\langle V_{3}, V_{3}\right\rangle=-1
$$

First, let

$$
\kappa_{1}=\frac{1}{\sqrt{-\left\langle V_{1}, V_{1}\right\rangle}}=\frac{1}{\sqrt{1-r^{2} s^{2}-t^{2}}},
$$

so that $\left\langle V_{1}, V_{1}\right\rangle=-1$. To make $\left\langle V_{j}, V_{j}\right\rangle=-1$ for $j=2,3$ we choose $\kappa_{2}$ so that $\left|\kappa_{2}\right|^{2}=\left(1-r^{2}\right)^{-1}$. Since $\kappa_{1} \in \boldsymbol{R}$ we have $\left\langle V_{1}, V_{2}\right\rangle=\left\langle V_{3}, V_{1}\right\rangle$. Setting $\left\langle V_{1}, V_{2}\right\rangle=\left\langle V_{2}, V_{3}\right\rangle$ and rearranging slightly we have

$$
\kappa_{2}^{3}=\kappa_{1}\left|\kappa_{2}\right|^{2} \frac{1+r^{2} s \sqrt{-1}}{1+r^{2}}=\kappa_{1} \frac{1+r^{2} s \sqrt{-1}}{1-r^{4}} .
$$

From Equation 13 and Equation 18 we compute that $\left|\kappa_{2}\right|^{6}=\left(1-r^{2}\right)^{-3}$, so that Equation 18 is compatible with the condition we placed on $\left|\kappa_{2}\right|^{2}$. Of the three possibilities for $\kappa_{2}$, we choose so as to make $V_{2}$ as high as possible, in the sense of $\S 2$. (We only care about this choice when $n=7$.)

Now we define some auxilliary elements. Let

$$
J\left(z_{1}, z_{2}, z_{3}\right)=\left(-\bar{z}_{1}, \bar{z}_{2}, \bar{z}_{3}\right) .
$$

From Equation 10 we can see that

$$
J\left(V_{1}, V_{2}, V_{3}\right)=\left(V_{1}, V_{3}, V_{2}\right)
$$

We define $J^{\prime}$ to be the unique linear transformation such that

$$
J^{\prime}\left(V_{1}, V_{2}, V_{3}\right)=\left(V_{2}, V_{3}, V_{1}\right) .
$$

It follows from Equation 16 that $J^{\prime} \in S U(2,1)$.

We now define an element which turns out to be extremely important for our purposes:

$$
K=-I_{2} \circ\left(J^{\prime}\right)^{-1} .
$$

In $\S 11.2$ we give an approximate numerical value for $K$, in the case $n=7$. The minus sign is introduced for technical purposes which have to do with the construction in $\S 4$. 
Lemma 3.1 We have the following relations:
1. $K \circ I_{1} \circ K^{-1}=I_{2} I_{3} I_{2}$.
2. $K \circ I_{2} \circ K^{-1}=I_{2} I_{1} I_{2}$.
3. $K \circ I_{3} \circ K^{-1}=I_{2}$.
4. $K^{3}=-I_{2} I_{1} I_{3}$.
5. $J \circ K \circ J^{-1}=K^{-1}$.

Proof: Recall from $\S 2.2$ that the negative eigenspace of a complex reflection is the one corresponding to the eigenvector -1 . By construction, the -1 eigenspace of $I_{j}$ is spanned by $V_{j-1}$ and $V_{j+1}$.

For the first relation, we observe that $I_{2} I_{3} I_{2}$ is a complex reflection whose negative eigenspace is spanned by $I_{2}\left(V_{1}\right)=-V_{1}$ and $I_{2}\left(V_{2}\right)$. On the other hand, $K I_{1} K^{-1}$ is a complex reflection whose negative eigenspace is spanned by $K\left(V_{2}\right)$ and $K\left(V_{3}\right)$. Using the definition of $K$, we have

$$
K\left(V_{2}\right)=-I_{2}\left(V_{1}\right)=V_{1} ; \quad K\left(V_{3}\right)=-I_{2}\left(V_{2}\right) .
$$

The two complex reflections in question have the same fixed sets. Hence they coincide. The second and third relations have essentially the same proofs.

For the fourth relation, we use the first three relations to compute that

$$
K^{3} \circ I_{j} \circ K^{-3}=\left(I_{2} I_{1} I_{3}\right) \circ I_{j} \circ\left(I_{2} I_{1} I_{3}\right)^{-1} .
$$

This implies that $K^{3}$ maps that points $\left[V_{j}\right] \in \boldsymbol{C H}^{2}$ to the point $I_{2} I_{1} I_{3}\left(\left[V_{j}\right]\right)$. Hence, there is some constant $\lambda_{j}$ such that $K^{3}\left(V_{j}\right)=\lambda_{j} I_{2} I_{1} I_{3}\left(V_{j}\right)$. We compute explicitly that $K^{3}\left(V_{2}\right)=-I_{2}\left(V_{2}\right)=-I_{2} I_{1} I_{3}\left(V_{2}\right)$. Hence $\lambda_{2}=-1$. Similar calculations show that $\lambda_{1}=\lambda_{3}=-1$. Hence $K$ and $-I_{2} I_{1} I_{3}$ agree on a basis.

The fifth relation follows from symmetry. For a direct calculation, we have

$$
\begin{gathered}
K^{-1}\left(V_{1}\right)=-J^{\prime}\left(I_{2}\left(V_{1}\right)\right)=J^{\prime}\left(V_{1}\right)=V_{2} ; \\
J\left(K\left(J^{-1}\left(V_{1}\right)\right)\right)=-J\left(K\left(V_{1}\right)\right)=J I_{2} J^{\prime-1}\left(V_{1}\right)=J I_{2}\left(V_{3}\right)=J\left(V_{3}\right)=V_{2}
\end{gathered}
$$

Similar calculations show that the two sides of the relation have the same action on $V_{2}$ and $V_{3}$. 


\subsection{Proof of Theorem 1.2}

We begin with a result which is probably well known:

Lemma 3.2 Let $H$ be a group generated by complex reflections $\gamma_{1}, \gamma_{2}, \gamma_{3}$ such that $\gamma_{i} \gamma_{j}$ has finite order $p_{i j} \in \boldsymbol{N}$ for all $i \neq j$. If $p_{12}=2$ then $H$ preserves a real slice $\Pi$ and $\left.H\right|_{\Pi}$ is the $\left(p_{12}, p_{23}, p_{31}\right)$-reflection triangle group.

Proof: Let $l_{j}$ be the complex slice fixed by $\gamma_{j}$. Let $x_{i j}=l_{i} \cap l_{j} \in \boldsymbol{C H}^{2}$ be the fixed point of the element $\gamma_{i} \gamma_{j}$. We normalize by an isometry so that $x_{12}=(0,0)$ and $x_{23}=(0, t)$ for some $t \in(0,1)$. Then $l_{2}$, which contains these two points, is the complex slice $\{(z, 0)\}$. Since $p_{12}=2$ the two slices $l_{1}$ and $l_{2}$ are perpendicular. There is a unique slice perpendicular to $l_{2}$ and containing $(0,0)$, namely $l_{1}=\{(0, w)\}$. The point $z_{13} \in l_{1}$ has the form $(0, \alpha)$ for some $\alpha \in \boldsymbol{C}$. We can further normalize by an isometry of the form $(z, w) \rightarrow(z, w u)$, where $u$ is unit complex, to arrange that $\alpha \in(0,1)$.

We have normalized so that all three points $x_{i j}$ are contained in $\boldsymbol{R}^{2}$. It follows from symmetry that $l_{k}$ is perpendicular to the real slice $\Pi=\boldsymbol{R}^{2} \cap \boldsymbol{C H}^{2}$. Hence, the restriction of $\gamma_{j}$ to $\Pi$ is a reflection in the real geodesic $\lambda_{j}=l_{j} \cap \Pi$. The angle between $\lambda_{i}$ and $\lambda_{j}$ is the same as the angle between $l_{i}$ and $l_{j}$, since $l_{i}$ and $l_{j}$ are perpendicular to $\Pi$. This forces $\left.H\right|_{\Pi}$ to be the $\left(p_{12}, p_{23}, p_{31}\right)$ reflection triangle group.

For pairwise unequal indices $i, j, k \in\{1,2,3\}$, let $H_{j k} \subset \Gamma(4, n)$ be the subgroup generated by the three complex reflections

$$
\gamma_{1}=I_{k} I_{i} I_{k} ; \quad \gamma_{2}=I_{i} ; \quad \gamma_{3}=I_{j}
$$

$\gamma_{1} \gamma_{2}=I_{i} I_{k} I_{i} I_{k}$ has order 2 and $\gamma_{2} \gamma_{3}=I_{i} I_{j}$ has order 4 and $\gamma_{1} \gamma_{3}=I_{k} I_{i} I_{k} I_{j}$ has order $n$. By Lemma 3.2, $H_{j k}$ stabilizes a real slice $\Pi_{j k}$ and acts as the $(2,4, n)$-reflection triangle group on $\Pi_{j k}$. The points $\left[V_{j}\right]$ and $\left[V_{k}\right]$, respectively, are the fixed points of $\gamma_{1} \gamma_{2}$ and $\gamma_{2} \gamma_{3}$. Hence

$$
\left[V_{j}\right],\left[V_{k}\right], I_{j}\left(\left[V_{j}\right]\right) \in \Pi_{j k} .
$$

Let $\Pi_{j k}^{\infty}$ be the $\boldsymbol{R}$-circle which is the ideal boundary of $\Pi_{j k}$. Since $\Pi_{j k} / H_{j k}$ is compact, $\Pi_{j k}^{\infty} \subset \Lambda(4, n)$. Let $\Upsilon=\bigcup \Pi_{i j}$ and $\Upsilon^{\infty}=\bigcup \Pi_{i j}^{\infty}$. The orbit

$$
\Gamma \Upsilon^{\infty}=\left\{g\left(\Upsilon^{\infty}\right) \mid g \in \Gamma(4, n)\right\}
$$


is a countable invariant union of $\boldsymbol{R}$-circles which is contained in $\Lambda(4, n)$. Since $\Lambda(4, n)$ is the minimal $\Gamma(4, n)$-invariant set, $\Gamma \Upsilon^{\infty}$ is dense in $\Lambda(4, n)$.

Equation 24 shows that $\Pi_{j k} \cap \Pi_{k j}$ contains two distinct points, and hence an entire geodesic. Therefore, $\Pi_{j k}^{\infty} \cap \Pi_{k j}^{\infty} \neq \emptyset$. Similarly, $\Pi_{j k}^{\infty} \cap \Pi_{j i}^{\infty} \neq \emptyset$. Since these two facts hold true for all relevant indices, $\Upsilon^{\infty}$ is connected. Since $I_{j}\left(\Upsilon^{\infty}\right) \cap \Upsilon^{\infty} \neq \emptyset$ we have that $\Upsilon^{\infty} \cup I_{j}\left(\Upsilon^{\infty}\right)$ is connected. Here $j=1,2,3$. It now follows, from induction on word length, that $\Gamma \Upsilon^{\infty}$ is connected.

It only remains to show that $\Gamma \Upsilon^{\infty}$ is nonplanar. The points $\left[V_{1}\right],\left[V_{2}\right], I_{2}\left[V_{2}\right]$ form a right angled geodesic triangle $T \subset \Pi_{12}$. Let $\gamma_{1}, \gamma_{2}, \gamma_{3}$ be the real geodesics extending the sides of $T$. The planes of $\Upsilon$ are all distinct, for otherwise $\Gamma(4,7)$ would stabilize a real slice, and this does not happen. It follows from this fact, and from symmetry, that there are real slices $S_{1}, S_{2}, S_{3} \subset \Gamma \Upsilon$, such that $S_{j} \cap \Pi_{12}=\gamma_{j}$. Figure 3.2 shows a set homeomorphic to the union $\Pi_{12}^{\infty} \cup S_{1}^{\infty} \cup S_{2}^{\infty} \cup S_{3}^{\infty}$. The hexagon represents $\Pi_{12}^{\infty}$. The drawing contains the complete bipartite graph $K_{3,3}$ and hence is not planar.

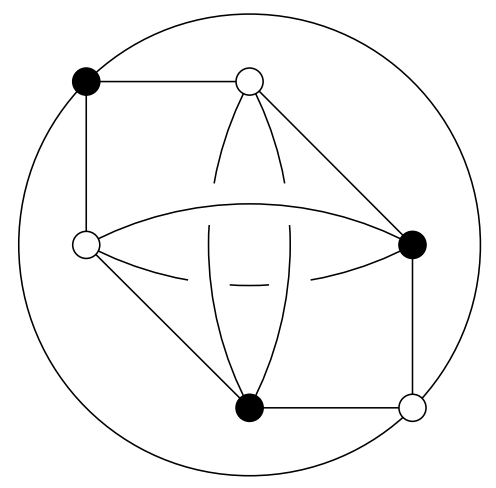

Figure 3.2

The accuracy of the picture depends on the fact that $S_{i}^{\infty} \cap S_{j}^{\infty}=\emptyset$, or equivalently, $S_{i} \cap S_{j}=\gamma_{i} \cap \gamma_{j}$. If this is false, then $S_{i} \cap S_{j}$ is a geodesic, and $S_{i} \cap S_{j} \cap \Pi_{12}$ is a single point. Translating this point to the origin in $\boldsymbol{C}^{2}$ we produce three totally real 2 dimensional subspaces of $\boldsymbol{C}^{2}$ which pairwise intersect in a line, but which triply intersect only at the origin. This is a well known impossibility.

Remark: It is not hard to deduce from what we have done that $\Lambda(4, n)$ is connected and locally connected, and that $\Lambda(4, n)$ has no planar neighborhoods. If we knew that $\Lambda(4, n)$ was 1 -dimensional (in the sense of $[\mathbf{A}]$ ) we could conclude from $[\mathbf{A}]$ that $\Lambda(4, n)$ is homeomorphic to the Menger curve. 


\subsection{Proof of Theorem 1.3}

Let $\tau_{3}=\operatorname{tr}\left(I_{1} I_{2} I_{3}\right)$ and $\tau_{4}=\operatorname{tr}\left(I_{1} I_{2} I_{1} I_{3}\right)$. Let $R=\boldsymbol{Z}\left[\tau_{3}, \bar{\tau}_{3}, \tau_{4}\right]$. We compute

$\tau_{4}=1+2 \cos (2 \pi / n) ; \quad \tau_{3}+\bar{\tau}_{3}=-2-2 \cos (2 \pi / n) ; \quad \tau_{3} \bar{\tau}_{3}=5+6 \cos (2 \pi / n)$.

Equation 26 tells us that every element of $R$ is an algebraic integer over $\boldsymbol{Z}[2 \cos (2 \pi / n)]$ and that $R$ is contained in a non-real quadratic field extension of $\boldsymbol{Q}[2 \cos (2 \pi / n)]$. Moreover, $R$ is closed under complex conjugation. Every $x \in R$ satisfies the polynomial $P(y)=(y-x)(y-\bar{x})=0$. The coefficients of this polynomial, $2 \Re(x)$ and $|x|^{2}$, both belong to $\boldsymbol{Z}[2 \cos (2 \pi / n)]$. To complete the proof of Theorem 1.3 we show that $\operatorname{tr}(\gamma) \in R$ for all $\gamma \in \Gamma(4, n)$.

Lemma 3.3 Let $A, X \in P U(2,1)$. If $\operatorname{tr}(A), \operatorname{tr}(X), \operatorname{tr}(A X), \operatorname{tr}\left(A^{-1} X\right) \in R$. then $\operatorname{tr}\left(A^{2} X\right) \in R$. If $\operatorname{tr}(A)=1$ and $\operatorname{tr}(X), \operatorname{tr}\left(A^{-1} X\right) \in R$ then $\operatorname{tr}(A X) \in R$ iff $\operatorname{tr}\left(A^{2} X\right) \in R$.

Proof: The characteristic polynomial of $A$ is $t^{3}-\operatorname{tr}(A) t^{2}+\overline{\operatorname{tr}}(A) t-1$. See [G, p. 206]. By the Cayley-Hamilton theorem, we can set $t=A$. We do this, then right-multiply the resulting equation by $A^{-1} X$, then take the trace of both sides. One gets $\operatorname{tr}\left(A^{2} X\right)=\operatorname{tr}(A) \operatorname{tr}(A X)-\overline{\operatorname{tr}}(A) \operatorname{tr}(X)+\operatorname{tr}\left(A^{-1} X\right)$. The lemma is obvious from this equation.

Say that $\gamma \in \Gamma(4, n)$ is critical if $\operatorname{tr}(\gamma) \notin R$ and if no shorter word has this property. We will suppose the existence of a critical word $\gamma$ and derive a contradiction. To streamline our notation we set $\gamma=i_{1} \ldots i_{m}$, provided that $\gamma=I_{i_{1}} \ldots I_{i_{m}}$. We write $\gamma_{1} \rightarrow \gamma_{2}$ to denote the sentence " $\gamma_{1}$ is critical implies $\gamma_{2}$ is critical."

Lemma 3.4 UijiV $\rightarrow U j i j V$ for arbitrary words $U$ and $V$.

Proof: Assume $U i j i V$ is critical. This word cannot be written in shorter form. It suffices to prove that $\operatorname{tr}(U j i j V) \notin R$. Define $A=i j$ and $X=i V U$. Note that $\operatorname{tr}(A)=1$. Obviously, $U i j i V \rightarrow i j i V U$, so that $\operatorname{tr}(A X) \notin R$. Since $X$ and $A^{-1} X=j V U$ are shorter than $U i j i V$, we get $\operatorname{tr}\left(A^{-1} X\right), \operatorname{tr}(X) \in R$. By Lemma 3.3 we have $\operatorname{tr}\left(A^{2} X\right)=\operatorname{tr}(i j i j i V U) \notin R$. Since $i j i j i=j i j$ we have $\operatorname{tr}(U j i j V)=\operatorname{tr}(j i j V U)=\operatorname{tr}(i j i j i V U) \notin R$. 
If $\gamma$ is critical then any conjugate of $\gamma$ is also critical. Also, by Lemma 3.3, a critical word cannot have the reduced form $A^{2} X$. These observations imply that a critical word must contain the string ...kijik... Using the symmetry of the generators, and conjugating, we can assume that our critical word begins $31213 \ldots$. Note that this word cannot end in a 3 . We will use the notation $Y$ ! to mean that $Y$ obviously cannot be critical. Here are the nontrivial reductions. We have underlined portions of our words, to indicate applications of Lemma 3.4 .

$\bullet 31213$ !

$\bullet 312 \underline{131} \rightarrow 312313$ !

$\bullet 3121312 \rightarrow(312)^{2} 1$ !

- $31213121 \ldots=(3121)^{2} \ldots$ !

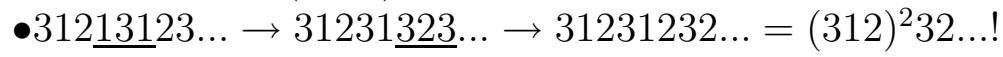

$\bullet 3121313 \ldots \rightarrow(13)^{2} \ldots$ !

$\bullet 3 \underline{12132} \rightarrow 321 \underline{232} \rightarrow 321323$ !

$\bullet 3121321 \rightarrow(213)^{2} 1$ !

- $3 \underline{121} 3212 \ldots \rightarrow 32123212 \ldots=(3212)^{2} \ldots$ !

$\bullet 31213213 \ldots=31(213)^{2} \ldots$ !

$\bullet 3121323 \ldots \rightarrow 3212323 \ldots \rightarrow(23)^{2} \ldots$ !

In short, there is no critical word, and Theorem 1.3 is true.

\subsection{Proof of Corollory 1.4}

Let $\alpha_{n}=2 \cos (2 \pi / n)$. Define

$$
M=\left[\begin{array}{ccc}
1 & 0 & 0 \\
0 & 1 & 0 \\
0 & 0 & \alpha_{n}
\end{array}\right]
$$

Let $\Gamma_{M}=M \Gamma M^{-1}$. Note that $\Gamma_{M}$ preserves the Hermitian form $L$, where

$$
L(Z, W)=z_{1} \bar{w}_{1}+z_{2} \bar{w}_{2}-\alpha_{n}^{-2} z_{3} \bar{w}_{3}
$$

and the matrix coefficients for all elements of $\Gamma_{M}$ lie in a finite field extension $\boldsymbol{E}$ of $\boldsymbol{Q} \alpha_{n}$. Let $\rho$ be the extension to $\boldsymbol{E}$ of any nontrivial Galois automorphism of $\alpha_{n}$. For $n=5,6,7,8,12$, all nontrivial Galois conjugates of $\alpha_{n}$ are negative. Hence $\rho\left(\Gamma_{M}\right)$ preserves the definite Hermitian form $\rho(L)$. That is, the trace of any element of $\Gamma(4, n)$ is an algebraic integer, all of whose nontrivial Galois conjugates are uniformly bounded. Indiscrete groups cannot have this property. 


\section{The View from the Outside}

\subsection{Overview}

We use the notation from $\S 3.1$ and $\S 3.2$. For the rest of the paper we concern ourselves only with the case $n=7$ considered in $\S 3.1$ and $\S 3.2$. We define

$$
Z=\bigcup_{m=-\infty}^{\infty} A(m) \cup B(m)
$$

Here $A(m)$ and $B(m)$ are finite unions of tetrahedra in $\boldsymbol{C}^{2,1}$. We call them pieces. The pieces have the property that $A(k+2 m)=K^{m}(A(k))$ and $B(k+2 m)=K^{m}(B(k))$ for all $k$ and $m$. Thus, $K(Z)=Z$. Here $K$ is the element defined in Equation 22. The tetrahedra in each piece are convex hulls of various 4-element subsets of their vertices. To take these convex hulls we only use the real affine structure of $\boldsymbol{C}^{2,1}$. The main object of study in this chapter is $\left[Z_{0}\right]=[Z] \cap S^{3}$, which turns out to be an infinite tiled cylinder.

To help understand the combinatorial structure of the tiling on $\left[Z_{0}\right]$ we will plot (portions of) $\left[Z_{0}\right]$ in two coordinate systems, as we now explain. Let $E_{0} K$ and $E_{\infty} K$ be the null eigenvectors of the element $K$, normalized to have third coordinate 1 . See $\S 10.2$ for numerical approximations. A positive eigenvector of $K$ is given by

$$
E_{+} K=E_{0} K \otimes E_{\infty} K
$$

where $\otimes$ is as in Equation 8. Given any point $[X] \in S^{3}-\left[E_{0} K\right]-\left[E_{\infty} K\right]$ we choose some lift $X \in N_{0}$ and define

$$
\Psi([X])=\left(\arg \frac{\left\langle X, E_{+} K\right\rangle}{\sqrt{\left\langle X, E_{0} K\right\rangle\left\langle X, E_{\infty} K\right\rangle}}, \frac{1}{2} \log \left|\frac{\left\langle X, E_{0} K\right\rangle}{\left\langle X, E_{\infty} K\right\rangle}\right|\right) .
$$

Our definition of $\Psi(X)$ is independent of lift of $[X]$. The domain of $\Psi$ is $S^{3}-\left[E_{0} K\right]-\left[E_{\infty} K\right]$ and the range is the flat cylinder $\boldsymbol{R} / 2 \pi \boldsymbol{Z} \times \boldsymbol{Z}$. We discussed $\Psi$ in detail in $[\mathbf{S 1}]$ and $[\mathbf{S 2}]$, where we called it the elevation map. In particular we proved that $\Psi$ has a globally well-defined branch.

Figure 4.1 shows the a large portion of $\exp \circ \Psi\left(\left[Z_{0}\right]\right)$. The tiling on $\left[Z_{0}\right]$ is actually a refinement of what is shown in Figure 4.1, and the grey lines of Figure 4.1 are images of lines of symmetry rather than edges of the tiling. The edges of the refinement, which we have hidden, are shown in Figures 4.4, 4.6 and 4.8 . 


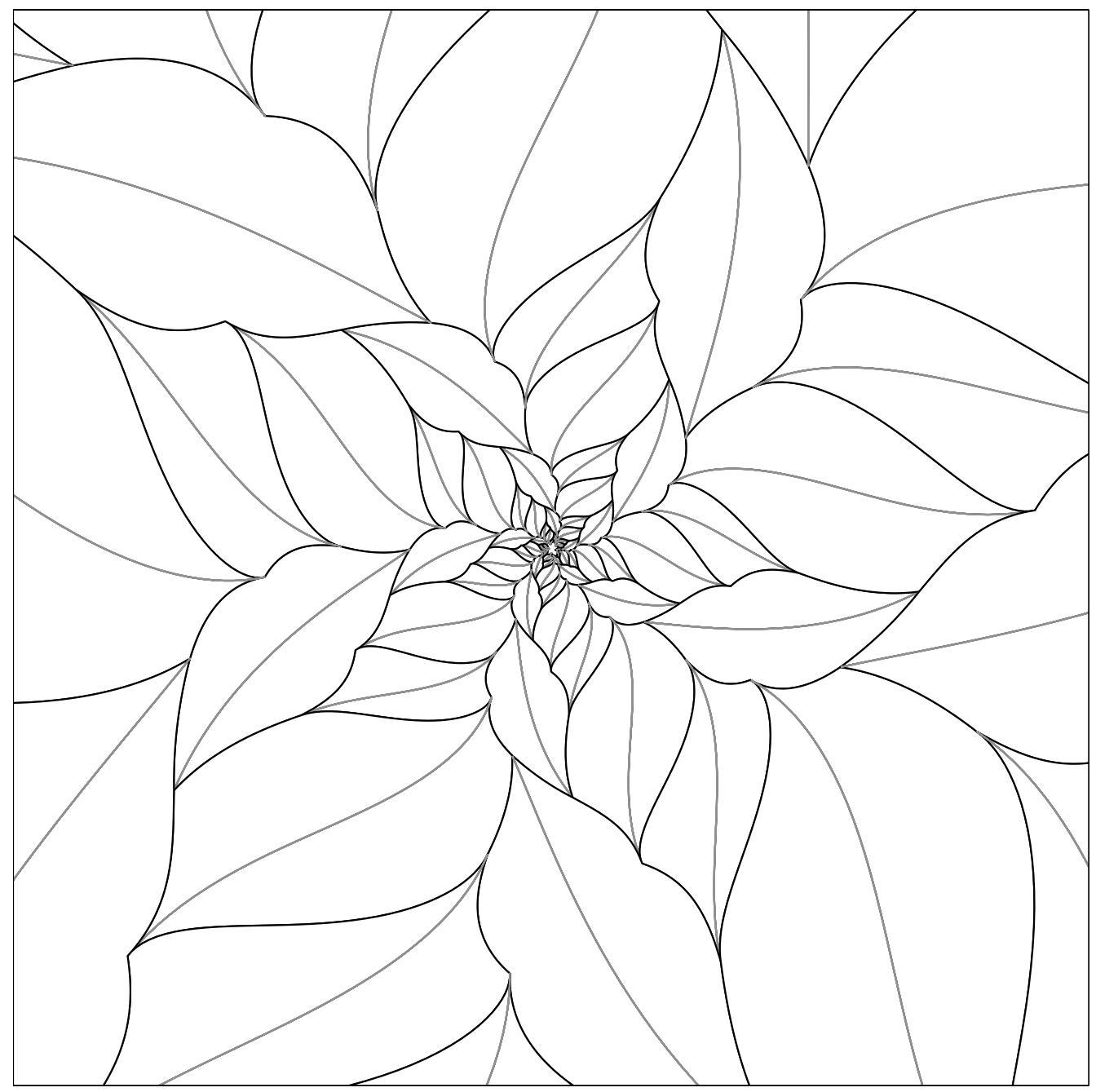

Figure 4.1

Compare Figure 4.1 with Figure 4.9 , which shows $\Psi\left(\left[Z_{0}\right]\right)$. The picture is combinatorially equivalent to Figure 4.1, but the labels of the pieces have been added in. The goal of this chapter is to build up to Figures 4.1 and 4.9.

Remark: In this paper $\Psi$ is just used to draw pictures. Our proofs do not use the structure of $\Psi$. However, $\Psi$ has a symmetry property which makes the pictures look nice: $\Psi$ conjugates the $P U(2,1)$-centralizer of $K$ to the translation group of the flat cylinder $\boldsymbol{R} / 2 \pi \boldsymbol{Z} \times \boldsymbol{R}$. 


\subsection{Notation}

If $g$ is an elliptic element of $\Gamma(4,7)$, and has a unique fixed point in $\boldsymbol{C H}^{2}$, we let $[g]$ be this fixed point. If $h$ is a hyperbolic element, we let $[h]$ denote the fixed point of $h$ which lies in $\left[N_{+}\right]$. We call this the word notation. In the word notation we have

$$
\left[V_{1}\right]=[23] ; \quad\left[V_{2}\right]=[31] ; \quad\left[V_{3}\right]=[12] .
$$

Here $V_{j}$ is as in Equation 10. We have the general principle that a word and its reverse denote the same point. Thus $[12]=[21]$ and $[1213]=[3121]$, etc.

It is easy to write down the action of the generators $I_{1}, I_{2}, I_{3}$ of $\Gamma(4,7)$ in this notation. The element $I_{j}$ takes the point $\left[a_{1}, \ldots, a_{n}\right]$ to the point $\left[j a_{1}, \ldots, a_{n} j\right]$. If the symbol $j j$ appears at either end, it is simply omitted. For instance, $I_{1}([1213])=[112131]=[2131]=[1312]$.

Let $J$ and $K$ be the extra elements defined in $\S 3.2$. If $w=[\ldots]$ is some fixed point, then the $J(w)$ is obtained by making the substitution $2 \rightarrow 3$ and $3 \rightarrow 2$. For instance $J([1213])=[1312]$. It follows from Lemma 3.1 that $K(w)$ is obtained by making the substitutions

$$
1 \rightarrow 232 \quad 2 \rightarrow 212 \quad 3 \rightarrow 2
$$

For instance $K([12])=[232212]=[2312]$. It follows from the fifth relation of Lemma 3.1 that $K^{-1}(w)$ is obtained by making the substitition

$$
1 \rightarrow 323 \quad 3 \rightarrow 313 \quad 2 \rightarrow 3
$$

For instance $K^{-1}([12])=[3233]=[32]$.

The vertices of our complex $Z$ are certain lifts of the $K$-orbits of the 4 points

$$
\text { [12]; [121312]; [1213121312]; [12131213121312]; }
$$

The first two of these points lie in $\boldsymbol{C H}^{2}$. The last two lie in $\left[N_{+}\right]$.

Using Equations 33 and 34 we can work out these orbits. We arrange them in a table, shown below. The action of $K$ maps each word to the one below it in the same column. The action of $J$ reverses each column, about the middle of the column. 


\begin{tabular}{|c|c|c|c|c|}
\hline & - & • & ○ & ○ \\
\hline-9 & & [32123213] & [31323132313213] & \\
\hline-8 & [31213213] & & & [31323123212323] \\
\hline-7 & & [131213] & [321232123213] & \\
\hline-6 & [312313] & & & [321231213121] \\
\hline-5 & & [313232] & [1312131213] & \\
\hline-4 & [3123] & & & [2313231323132131] \\
\hline-3 & & [1213] & [3132313232] & \\
\hline-2 & [13] & & & [32321232123212] \\
\hline-1 & & [3132] & [12132123] & \\
\hline 0 & [23] & & & [12131213121312] \\
\hline 1 & & [2123] & [13123132] & \\
\hline 2 & {$[12]$} & & & [23231323132313] \\
\hline 3 & & {$[1312]$} & [2123212323] & \\
\hline 4 & [2132] & & & [3212321232123121] \\
\hline 5 & & {$[212323]$} & [1213121312] & \\
\hline 6 & [213212] & & & [231321312131] \\
\hline 7 & & [121312] & [231323132312] & \\
\hline 8 & {$[21312312]$} & & & [21232132313232] \\
\hline 9 & & [23132312] & [21232123212312] & \\
\hline
\end{tabular}

The middle of the last column looks like it is not symmetric with respect to $J$. However, the relation $\left(I_{1} I_{2} I_{1} I_{3}\right)^{7}=e$ implies that

$$
[12131213121312]=[13121312131213]
$$

so the chart is symmetric after all.

As an alternate system of notation, we let $[\bullet j]$ be the vector which is in one of the first two columns, and in the $j$ th row. For instance $[\bullet 4]=[2132]$ and $[\bullet 3]=[1312]$. We let $[\circ j]$ be the vector which is in one of the last two columns and in the $j$ th row. We will use the notation $[* j]$ when it does not matter if we are speaking about $[\bullet j]$ or $[\circ j]$. We call this the chart notation. It follows immediately from our definitions that

$$
J([* j])=[*(-j)] ; \quad K([* j])=[*(j+2)] .
$$

By construction $[\bullet j] \in\left[N_{-}\right]=\boldsymbol{C H}^{2}$ and $[\circ j] \in\left[N_{+}\right]$. 


\subsection{Canonical Lifts}

In this section we construct a canonical lift for $[* j]$. We call this lift $* j$. We require that

$$
\langle\circ j, \circ j\rangle=-\langle\bullet j, \bullet j\rangle=1 . \quad J(* j)=*(-j) ; \quad K(* j)=*(j+2) ;
$$

Compare Equation 36.

We claim that Equation 37 determines our lifts uniquely, up to sign. To see this, suppose for example that $P_{1}$ and $P_{2}$ are both lifts of $* 0$. We have $P_{2}=\lambda P_{1}$, where $|\lambda|=1$. From equation 19 and $J\left(P_{2}\right)=P_{2}$ we have

$$
\lambda P_{1}=J\left(\lambda P_{1}\right)=\bar{\lambda} J\left(P_{1}\right)=\bar{\lambda} P_{1} .
$$

Hence $\lambda= \pm 1$. At the same time, we can take any lift $P_{1}$ of $* 0$ which satisfies the first equation in Equation 37, and then adjust $\lambda$ so that $\lambda P_{1}$ satisfies the second one as well. We can play a similar game for $* 1$, using the symmetry $K \circ J(* 1)=* 1$ to determine $\lambda$. We get the remaining lifts, up to sign, using the action of $K$.

It remains to determine the signs. Referring to Equation 10, we note that $V_{1}$ satisfies all the requirements for $\bullet$. Thus we set $\bullet 0=V_{1}$. A calculation shows that this choice of sign makes the lift of [12] high, in the sense of $\S 2$. For the remaining vectors we choose the signs so that the lifts of the points of Equation 35 are high. See $\S 10.2$ for numerical values.

\subsection{The Join Construction}

We think of $\boldsymbol{C}^{2,1}$ as an affine space. If $S_{1}, S_{2} \subset \boldsymbol{C}^{2,1}$ are disjoint subsets of $C^{2,1}$, the join $S_{1} \bowtie S_{2}$ is defined as the union of all line segments connecting a point in $S_{1}$ to a point in $S_{2}$. For example, the join of two general position line segments is a tetrahedron. In our constructions below it will be useful to call $S_{1}$ the axis and $S_{2}$ the rim. See the pictures below.

Given a finite list of points $P_{1} \ldots P_{k}$ we let

$$
\left[P_{1}, \ldots, P_{k}\right]=\left(P_{1} \bowtie P_{2}\right) \cup\left(P_{2} \bowtie P_{3}\right) \ldots \cup \ldots\left(P_{k-1} \bowtie P_{k}\right)
$$

$\left[P_{1} \ldots P_{k}\right]$ is an open polygonal path in $\boldsymbol{C}^{2,1}$. We define the closed polygonal path

$$
\left[P_{1} \ldots P_{k}\right]_{\square}=\left(P_{k} \bowtie P_{1}\right) \cup\left[P_{1} \ldots P_{k}\right]
$$




\subsection{The Odd B Pieces}

When $j$ is odd we define

$$
B(j)=[\bullet(j-3) \bullet(j+3)] \bowtie[\bullet(j-2) \bullet(j-1) \bullet(j+1) \bullet(j+2)]_{\square} .
$$

Figure 4.1 shows $B(1)$. We label the points using both notations.

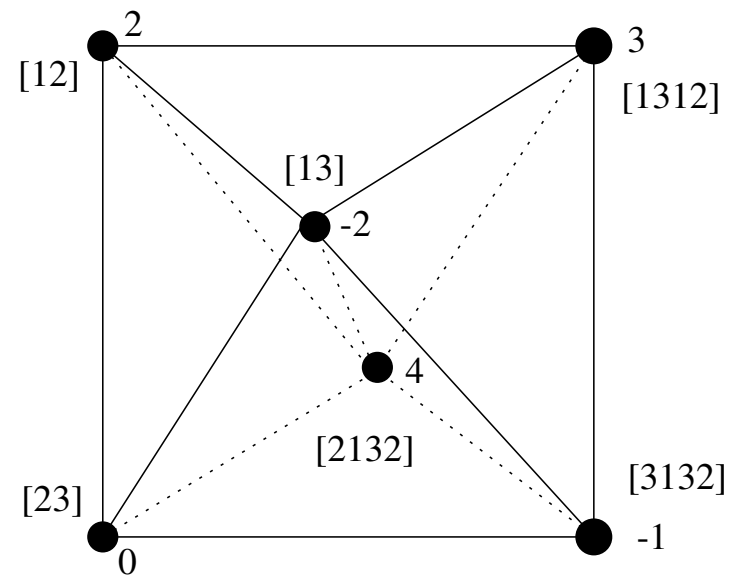

Figure 4.2

Lemma $4.1 K \circ J(B(1))=B(1)$ and $I_{2}(B(1))=-B(1)$.

Proof: The first equation is clear from Equation 37 and from the chart notation in Figure 4.2. In the second equation we mean that the action of $I_{2}$ multiplies each vertex of $B(1)$ by -1 . Looking at the word notation in Figure 4.2 , it is easy to see that $I_{2}$ fixes the projectivizations of the points on the rim of $B(1)$. Thus, the 4 points on the rim are all eigenvectors of $I_{2}$. These 4 points are all negative vectors, so they lie in the negative eigenspace of $I_{2}$. We now prove $I_{2}(\bullet 4)=-\bullet(-2)$. This is the same as proving that

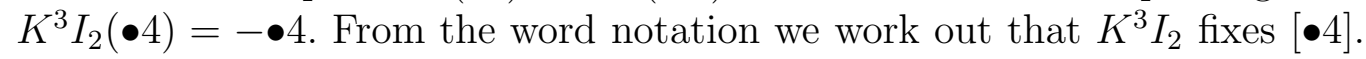
Hence $\bullet 4$ is an eigenvector of $K^{3} I_{2}=-I_{2} I_{1} I_{3} I_{2}$. This element has eigenvalues \pm 1 . An easy calculation shows that $K^{3} I_{2}$ does not fix $\bullet 4$. Hence, the eigenvalue corresponding to $\bullet 4$ is -1 . Since $I_{2}$ is an involution we get $I_{2}(\bullet(-2))=-\bullet 4$ as well.

Similar results hold for the other odd $B$ pieces. 


\subsection{The Even A Pieces}

When $j$ is even we define

$$
\begin{array}{r}
A(j)=[\bullet(j-3) \bullet(j+3)] \bowtie \\
{[\bullet(j-2) \bullet(j-7) \circ(j-5) \circ j \circ(j+5) \bullet(j+7) \bullet(j+2)]_{\square}}
\end{array}
$$

Figure 4.3 shows $A(0)$.

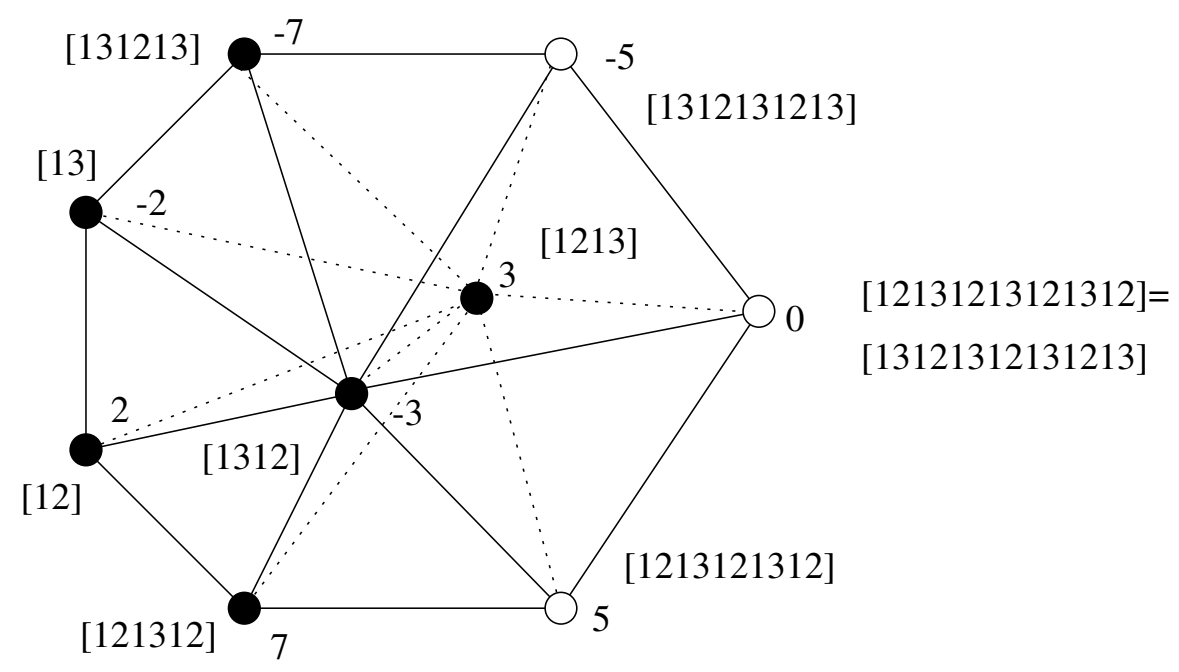

Figure 4.3

Lemma 4.2 $J(A(0))=A(0)$ and $I_{1}(A(0))=-A(0)$.

Proof: The first equality is obvious from Equation 37 and the chart notation in Figure 4.3. For the second equality, it is easy to see that the 7 vectors in the rim are eigenvectors of $I_{1}$. The negative vectors on the rim must lie in the negative eigenspace of $I_{1}$ and (as shown by a direct computation) so do the positive ones. It remains to show that $\bullet 3$ is an eigenvector of $K^{3} I_{1}=-I_{2} I_{1} I_{3} I_{1}$ corresponding to the eigenvalue -1 . The argument is similar to the one given in Lemma 4.1.

Figure 4.4 shows $\Psi\left([A(0)] \cap S^{3}\right)$. Here is a heuristic explanation. If a tetrahedron $\tau$ of $A(0)$ has 3 negative vertices and one positive vertex, then one would expect (under suitable transversality hypotheses) that $\tau \cap S^{3}$ is an embedded triangle. If $\tau$ has 2 negative vertices and 2 positive vertices, one 
would expect that $\tau \cap S^{3}$ is an embedded quadrilateral. Looking at Figure 4.3 , we see that there are 4 tetrahedra in $A(0)$ which have both positive and negative vertices. Two of these are of the $3+1$ type and the other two are of the $2+2$ type. Thus, Figure 4.4 shows two triangles and two quadrilaterals. The first equality of Lemma 4.2 accounts for the rotation symmetry.

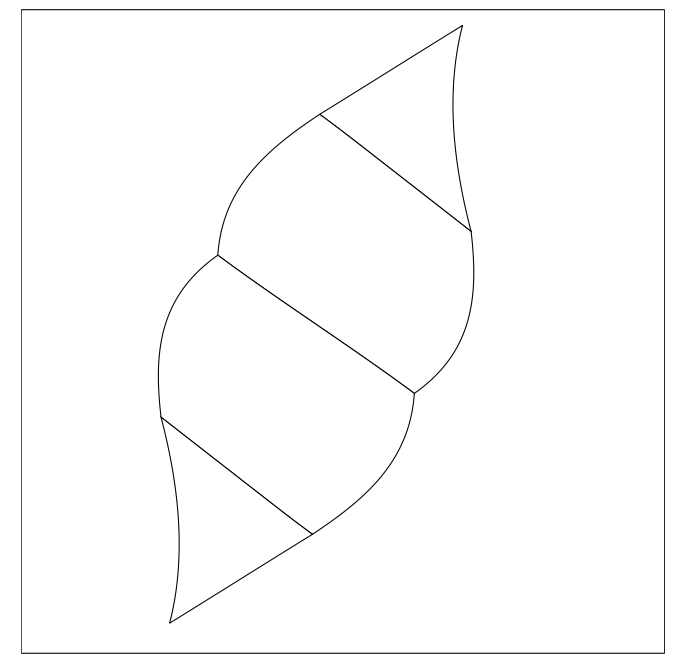

Figure 4.4

\subsection{The Even B Pieces}

When $j$ is even we define

$$
B(j)=[\circ(j-3) \circ(j+3)] \bowtie[\circ(j+8) \bullet(j+5) \bullet(j-5) \circ(j-8)]
$$

Figure 4.5 shows $B(0)$.

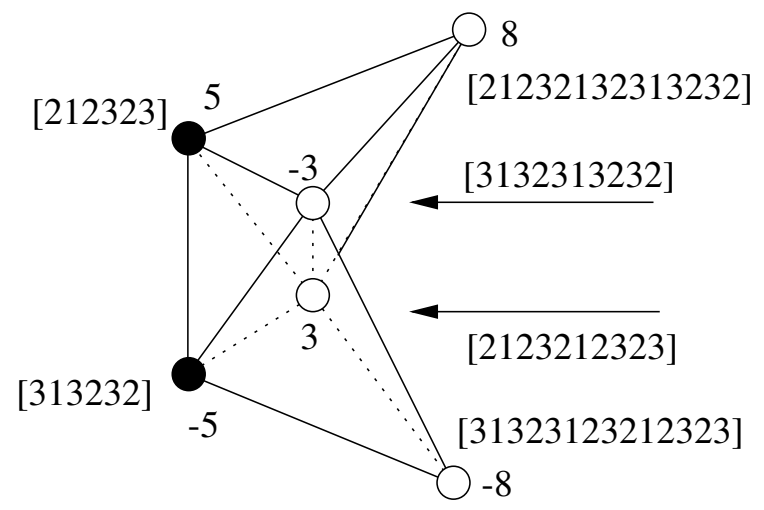

Figure 4.5 
Lemma 4.3 $J(B(0))=B(0)$ and $I_{2} I_{3} I_{2} I_{3} I_{1} I_{3} I_{2} I_{3} I_{2}(B(0))=-B(0)$.

Proof: The proof is essentially the same as in Lemmas 4.1 and 4.2. Let $I^{\prime}$ be the complex reflection of the lemma. We use the fact that $I_{2} I_{3} I_{2} I_{3}=I_{3} I_{2} I_{3} I_{2}$ and compute that $I^{\prime}$ fixes [•5]. We have

$$
\begin{gathered}
I^{\prime}([212323])=[232313232.212323 .323212323]= \\
{[232313232323]=[323212]=[\bullet 5] .}
\end{gathered}
$$

To show that $I^{\prime}$ fixes $[0(-8)]$ we compute

$$
\begin{aligned}
& I^{\prime}([31323123212323])=[232312323.31323123212323 .323213232] \\
& \quad=[23231232132312323232]=[23231232132313]=[\circ(-8)] .
\end{aligned}
$$

$I^{\prime}$ commutes with $J$ and hence fixes 08 and $\bullet(-5)$ by symmetry. The remaining calculations are similar to what we have already done.

Remark: The emacs word processor makes short work of calculations like the ones we have just done.

Figure 4.6 shows $\Psi\left([B(0)] \cap S^{3}\right)$.

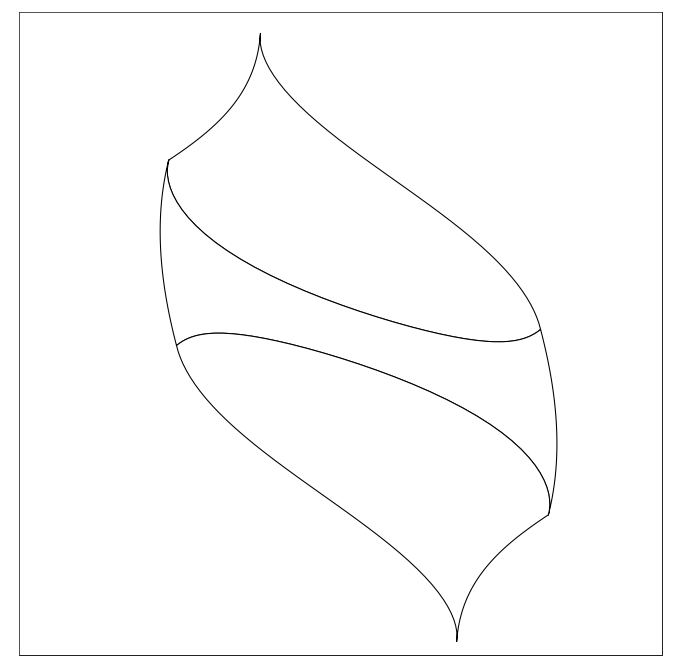

Figure 4.6 


\subsection{The Odd A Pieces}

When $j$ is odd we define

$$
A(j)=[\circ(j-3) \circ(j+3)] \bowtie[\circ(j-8) \bullet j \circ(j+8)] .
$$

Figure 4.7 shows $A(1)$.

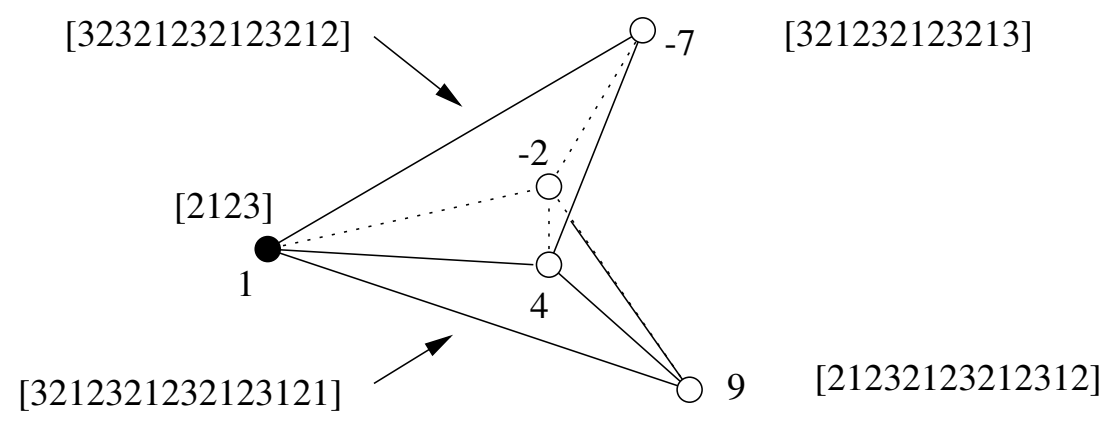

Figure 4.7

Lemma $4.4 K \circ J(A(1))=A(1)$ and

$I_{3} I_{2} I_{1} I_{2} I_{3} I_{2} I_{1} I_{2} I_{3} I_{2} I_{1} I_{2} I_{3}(A(1))=-A(1)$.

Proof: The proof is essentially the same as in Lemmas 4.1 and 4.2. However, one of the calculations is slightly nontrivial. Let $I^{\prime}$ be the complex reflection listed in the lemma. We compute

$$
\begin{gathered}
I^{\prime}([\circ(-2)])=[3212321232123323 \underline{212321232123212321232123}]= \\
{[3212321232123323 \underline{3212}]=[321232123 \underline{21212}]=[321232123 \underline{121}]=[\circ 4] .}
\end{gathered}
$$

The first equality comes from $T_{3} T_{2} T_{1} T_{2}=\left(T_{2} T_{1} T_{2} T_{3}\right)^{6}$; the second is cancellation; the third comes from $T_{2} T_{1} T_{2} T_{1} T_{2}=T_{1} T_{2} T_{1}$. This calculation shows that $\circ 4$ is an eigenvector of $K^{3} I^{\prime}$. An explicit calculation, using Lemma 3.3 for instance, shows that the only unit norm eigenvector of $K^{3} I^{\prime}$ is -1 . Since $\circ 4 \notin N_{0}$, it must belong to the corresponding eigenspace.

Figure 4.8 shows a plot of $\Psi\left([A(1)] \cap S^{3}\right)$. 


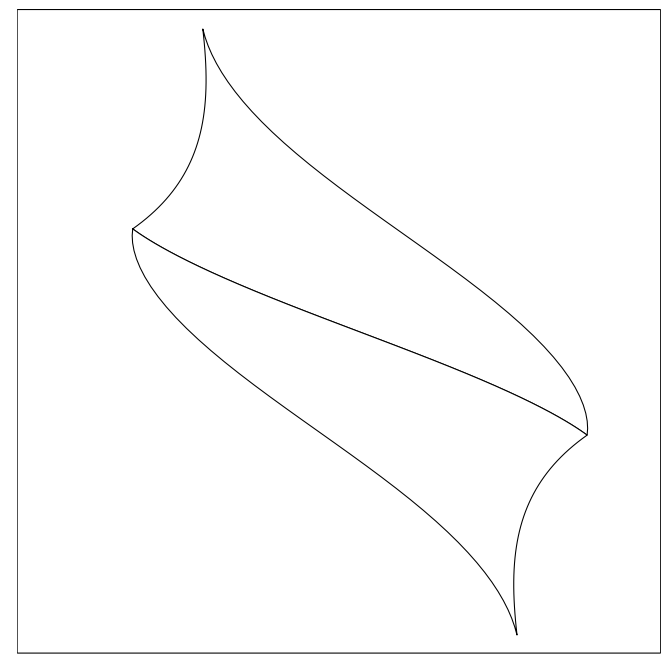

Figure 4.8

\subsection{The Whole Tiling}

By Lemmas 4.1-4.4, there are complex reflections $A_{m}$ and $B_{m}$, henceforth called reflection pairings, such that

$$
A_{m}([A(m)])=[A(m)] ; \quad B_{m}([B(m)])=[B(m)] .
$$

Explicitly:

$$
\begin{array}{r}
A_{2 m}=K^{m} I_{1} K^{-m} ; \quad A_{2 m+1}=K^{m} I_{3} I_{2} I_{1} I_{2} I_{3} I_{2} I_{1} I_{2} I_{3} I_{2} I_{1} I_{2} I_{3} K^{-m} \\
B_{2 m+1}=K^{m} I_{2} K^{-m} \quad B_{2 m}=K^{m} I_{2} I_{3} I_{2} I_{3} I_{1} I_{3} I_{2} I_{3} I_{2} K^{-m}
\end{array}
$$

Here is a table listing the first few reflection pairings. As in $\S 3.4$ we use the notation $\ldots i j \ldots$ to denote $\ldots I_{i} I_{j} \ldots$ The entries are computed using Lemma 3.1.

$\left|\begin{array}{ll|ll}A_{-4} & 131 & B_{-4} & 321232123 \\ A_{-3} & 12131213121 & B_{-3} & 313 \\ A_{-2} & 323 & B_{-2} & 1312131 \\ A_{-1} & 2313231323132 & B_{-1} & 3 \\ A_{0} & 1 & B_{0} & 232313232 \\ A_{1} & 3212321232123 & B_{1} & 2 \\ A_{2} & 232 & B_{2} & 1213121 \\ A_{3} & 13121312131 & B_{3} & 212 \\ A_{4} & 121 & B_{4} & 231323132\end{array}\right|$




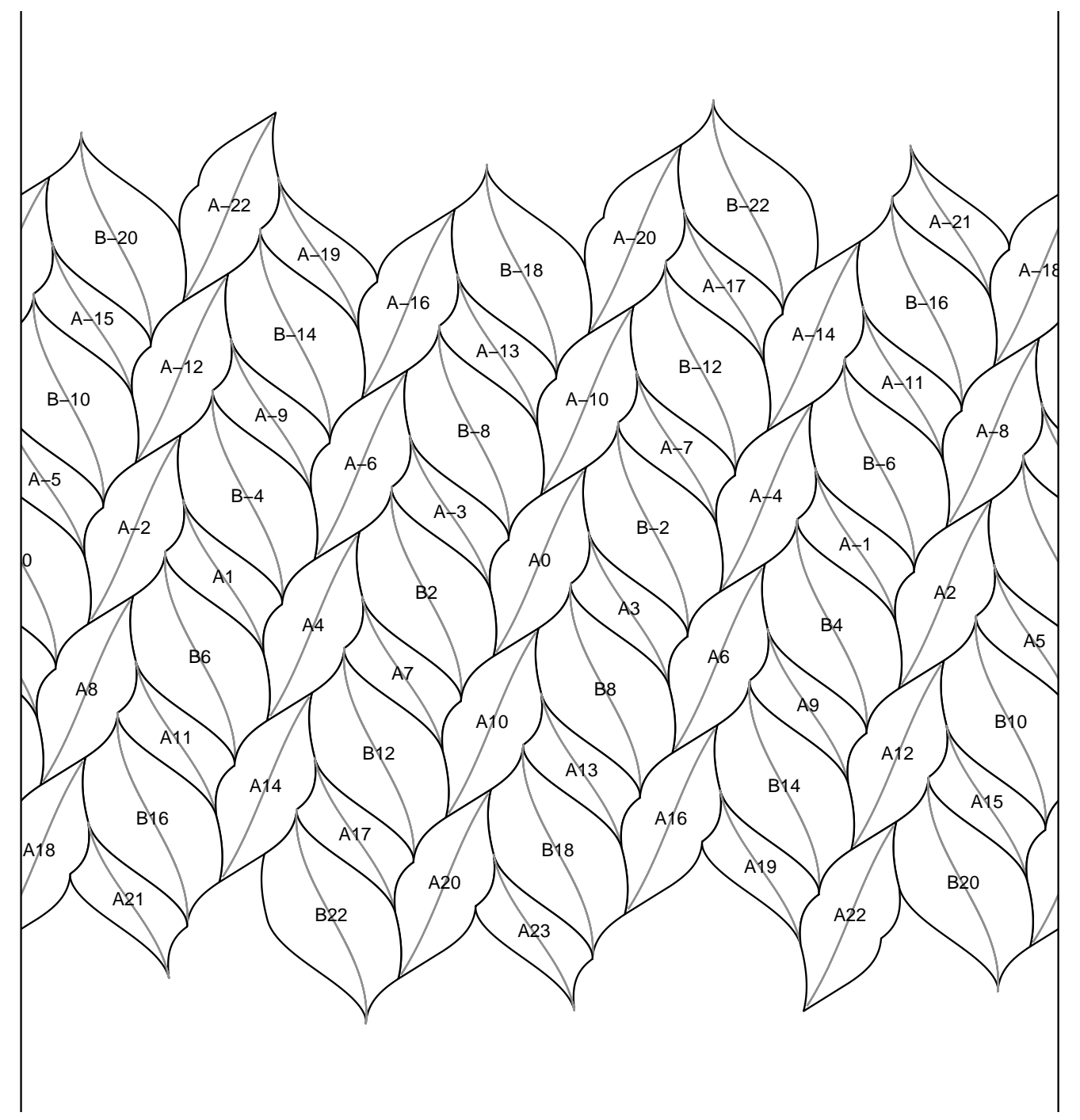

Figure 4.9

Figure 4.9 shows $\Psi\left(\left[Z_{0}\right]\right)$, with the tiles labelled. We have erased some of the lines in Figures 4.4, 4.6 and 4.8. The grey curves are images of $\boldsymbol{C}$ circles fixed by the reflection pairings. The vertical sides of the picture are identified, so that the plot takes place on a cylinder.

We finish this chapter with a result which plays a key role in our analysis of $\Omega(4,7) / \Gamma(4,7)$. 
Lemma 4.5 In $P U(2,1)$ we have the following relations:

1. $A_{k+2 m}=K^{m} A_{k} K^{-m}$

2. $B_{k+2 m}=K^{m} B_{k} K^{-m}$.

3. $A_{-2} B_{0} A_{2}=K^{3}$.

4. $B_{-2} A_{3} A_{0}=K^{3}$.

5. $A_{1} B_{6} A_{-2}=K^{3}$.

Proof: The first 2 relations are restatements of Equation 46. For the third relation:

$$
A_{-2} B_{0} A_{2}=323232313232232=323323 \underline{213232232}=213=-K^{3} .
$$

Here we used $2323=3232$ and, for the last equality, Lemma 3.1. So far we have computed in $S U(2,1)$. When we projectivize, the minus sign goes away. The fourth relation has a similar proof. For the fifth relation, we have

$$
B_{6}=213 B_{0} 312=21232123212 \text {. }
$$

Hence $A_{1} B_{6} A_{-2}$ expands as

$$
3212321232123.21232123212 .323=(3212)^{6} 323=2123323=213=-K^{3} .
$$

Here we used the fact that $(3212)^{7}$ is the identity. Again, the minus sign goes away when we projectivize. 


\section{The Hyperbolic Structure}

\section{$5.1 \quad$ Real Hyperbolic Space}

We represent real hyperbolic 3-space, $\boldsymbol{H}^{3}$, as the upper half space $\boldsymbol{C} \times \boldsymbol{R}^{+}$. We identify the ideal boundary of $\boldsymbol{H}^{3}$ with the Riemann sphere $\boldsymbol{C} \cup \infty$. There is a natural projection from $\boldsymbol{H}^{3}$ to $\boldsymbol{C}$ given by the map $\pi(z, t)=z$. We use this projection for many of the computer plots in this chapter.

The orientation preserving isometry group of $\boldsymbol{H}^{3}$ can be identified with the projective special linear group $P S L_{2}(\boldsymbol{C})$. The group $P S L_{2}(\boldsymbol{C})$ acts on $\boldsymbol{C} \cup \infty$ via Moebius transformations, in the usual way:

$$
\left[\begin{array}{ll}
a & b \\
c & d
\end{array}\right]: z \rightarrow \frac{a z+b}{c z+d}
$$

Such maps extend to give isometries of $\boldsymbol{H}^{3}$, though the formula for the extension is somewhat involved. Below we sometimes specify isometries of $\boldsymbol{H}^{3}$ by matrices in $G L_{2}(\boldsymbol{C})$, the general linear group, rather than by matrices in $S L_{2}(\boldsymbol{C})$. One can always renormalize these matrices to have determinant 1, so that they lie in $S L_{2}(\boldsymbol{C})$.

A loxodromic element has two fixed points on $\boldsymbol{C} \cup \infty$ and stabilizes the geodesic in $\boldsymbol{H}^{3}$ which has these two points as endpoints. For instance

$$
K_{*}=\left[\begin{array}{cc}
\lambda & 0 \\
0 & \lambda^{-1}
\end{array}\right] ; \quad|\lambda| \neq 1
$$

represents a loxodromic element whose fixed points are 0 and $\infty$.

A halfturn is an involution which rotates $\pi$ degrees around a geodesic. This fixed geodesic is called the axis of the halfturn. For instance

$$
J_{*}=\left[\begin{array}{ll}
0 & i \\
i & 0
\end{array}\right]
$$

represents a halfturn whose fixed points are \pm 1 . Halfturns are characterized by the property the matrices representing them have trace 0 .

We point out a certain analogy between halfturns and complex reflections. If we consider $\boldsymbol{H}^{3}$ as a subset of $S^{3}$, then the action of a halfturn naturally extends to give a conformal isomorphism of $S^{3}$. This conformal isomorphism is topologically conjugate to the action of a complex reflection on $S^{3}$. 


\subsection{Some Special Halfturns}

Let $K_{*}$ be as in Equation 48. In this section we will find, for all $m, n \in \boldsymbol{Z}$, halfturns $A_{n}^{*}, B_{2 m}^{*} \in G L_{2}(\boldsymbol{C})$ whose projectivizations satisfy all the relations of Lemma 4.5 (with the stars added). These halfturns will be used to define the infinite hyperbolic polyhedron whose projection is shown in Figure 5.6 below.

Given $|\lambda|>1$ and $a_{0}, b_{0}, a_{1} \in \boldsymbol{C}-\{ \pm 1\}$ we define the halfturn pairings

$$
\begin{gathered}
A_{2 n}^{*}=K_{*}^{n}\left[\begin{array}{cc}
a_{0} & -1 \\
1 & -a_{0}
\end{array}\right] K_{*}^{-n} ; \\
B_{2 n}^{*}=K_{*}^{n}\left[\begin{array}{cc}
b_{0} & -1 \\
1 & -b_{0}
\end{array}\right] K_{*}^{-n} ; \\
A_{2 n+1}^{*}=K_{*}^{n}\left[\begin{array}{cc}
a_{1} & -\lambda \\
\lambda^{-1} & -a_{1}
\end{array}\right] K_{*}^{-n} .
\end{gathered}
$$

Define

$$
\lambda_{n}=\operatorname{tr}\left(K_{*}^{n}\right)=\lambda^{n}+\lambda^{-n} .
$$

Lemma 5.1 The halfturn pairings satisfy the relations of Lemma 4.5 only if

$$
2 \lambda_{5}=\lambda_{7} \lambda_{8} ; \quad a_{0}^{2}=\frac{\lambda_{7}}{\lambda_{3}} ; \quad b_{0}^{2}=\frac{\lambda_{5}^{2}}{\lambda_{7} \lambda_{3}} ; \quad a_{1}^{2}=\frac{\lambda_{3}^{2} \lambda_{7}+\lambda_{5}^{2} \lambda_{7}-2 \lambda_{3} \lambda_{5}^{2}}{\lambda_{3} \lambda_{7}^{2}-\lambda_{3} \lambda_{5}^{2}}
$$

Proof: If the halfturn pairings satisfy the third relation of Lemma 4.5 then there is a constant constant $z_{1} \in \boldsymbol{C}$ such that

$$
K_{*}^{3}=z_{1} A_{-2}^{*} B_{0}^{*} A_{2}^{*}=z_{1} K_{*}^{-1} A_{0}^{*} K_{*} B_{0}^{*} K_{*} A_{0}^{*} K_{*}^{-1}
$$

Rearranging, we get

$$
z_{1} B_{0}^{*}=K_{*}^{-1} A_{0}^{*} K_{*}^{5} A_{0}^{*} K_{*}^{-1}=\left[\begin{array}{cc}
\lambda^{-7}-a_{0}^{2} \lambda^{3} & a_{0}\left(\lambda^{5}-\lambda^{-5}\right) \\
a_{0}\left(\lambda^{-5}-\lambda^{5}\right) & \lambda^{7}-a_{0}^{2} \lambda^{-3}
\end{array}\right]
$$

Call the matrix on the right $\mu$. Since $B_{0}^{*}$ is a halfturn, $\operatorname{tr}(\mu)=0$. This gives the equation for $a_{0}^{2}$. We have $b_{0}^{2}=\mu_{11} \mu_{22} / \mu_{12} \mu_{21}$. This gives the equation for $b_{0}^{2}$. 
If the halfturn pairings satisfy the fourth relation of Lemma 4.5 there is some constant $z_{2} \in \boldsymbol{C}$ such that

$$
K_{*}^{3}=z_{2} B_{-2}^{*} A_{3}^{*} A_{0}^{*}=z_{2} K_{*}^{-1} B_{0}^{*} K_{*}^{2} A_{1}^{*} K_{*}^{-1} A_{0}^{*} .
$$

Rearranging, we get

$$
z_{2} A_{1}^{*}=K_{*}^{-2} B_{0}^{*} K_{*}^{4} A_{0}^{*} K_{*}=\left[\begin{array}{cc}
a_{0} b_{0} \lambda^{3}-\lambda^{-5} & a_{0} \lambda^{-7}-b_{0} \lambda \\
-b_{0} \lambda^{-1}+a_{0} \lambda^{7} & a_{0} b_{0} \lambda^{-3}-\lambda^{5}
\end{array}\right]
$$

Call this last matrix $\nu$. Since $A_{1}^{*}$ is a halfturn, $\operatorname{tr}(\nu)=0$. This gives

$$
a_{0} b_{0}=\lambda_{5} / \lambda_{3} ; \quad b_{0} / a_{0}=\lambda_{5} / \lambda_{7}
$$

The second equation follows from the first and from the equation for $b_{0}^{2}$.

If the halfturn pairings satisfy the fifth relation of Lemma 4.5 then there is a constant $z_{3}$ such that

$$
z_{3} A_{1}^{*}=\eta=K_{*}^{2} A_{0}^{*} K_{*}^{4} B_{0}^{*} K_{*}^{-3} .
$$

We compute symbolically that

$$
\eta_{11} / \nu_{11}=\eta_{22} / \nu_{22}=1 ; \quad \eta_{21} / \nu_{21}=\frac{\lambda^{8}\left(b_{0}-a_{0} \lambda^{8}\right)}{a_{0}-b_{0} \lambda^{8}} ; \quad \eta_{12} / \nu_{12}=\nu_{21} / \eta_{21}
$$

Setting $\eta_{21} / \nu_{21}=1$ we get

$$
2 b_{0} / a_{0}=\lambda_{8}
$$

Plugging in the second equation from Equation 56 we get the equation for $\lambda$.

Using the equation $a_{1}^{2}=\nu_{11} \nu_{22} / \nu_{12} \nu_{21}$ we get the equation

$$
a_{1}^{2}=\frac{1+\left(a_{0} b_{0}\right)^{2}-a_{0} b_{0} \lambda_{8}}{a_{0}^{2}+b_{0}^{2}-a_{0} b_{0} \lambda_{8}}
$$

Plugging in the equations we already have for the quantities in the last equation, we get the equation for $a_{1}^{2}$ listed in the lemma.

Our derivation has one lucky accident. If our matrix $\nu$ is really a multiple of $A_{1}^{*}$ then we must have $\nu_{12}^{2}-\lambda^{4} \nu_{21}^{2}=0$. When we expand this out we get the equation

$$
0=\lambda^{-14}\left(a_{0}^{2}-2 a_{0} b_{0} \lambda^{8}+2 a_{0} b_{0} \lambda^{24}-a_{0}^{2} \lambda^{32}\right)=R(\lambda) H\left(\lambda_{2}\right) .
$$


$R(\lambda)$ is a rational function which factors into cyclotomic polynomials and $H$ is the polynomial

$$
H(z)=z^{6}+z^{5}-4 z^{4}-3 z^{3}+4 z^{2}-2
$$

The lucky accident is that $H\left(\lambda_{2}\right)$ and $2 \lambda_{5}-\lambda_{7} \lambda_{8}$ agree modulo cyclotomic polynomials. Thus, what could have been a second equation for $\lambda$ turns out to be the same equation.

One of the roots of $2 \lambda_{5}-\lambda_{7} \lambda_{8}$ is within $10^{-15}$ of $\lambda^{\prime}$, where

$$
\lambda^{\prime}=-0.33965052546914795+1.0311113175790407 \sqrt{-1} .
$$

Our choice of $\lambda$ plugs into the other equations to determine $a_{0}^{2}, b_{0}^{2}$ and $a_{1}^{2}$. These numbers have square roots which are within $10^{-5}$ of the numbers

$$
\begin{gathered}
a_{0}^{\prime}=1.0408951341934188+0.29367056890017413 \sqrt{-1} \\
b_{0}^{\prime}=-1.1705635976637505+0.1147136622789418 \sqrt{-1} \\
a_{1}^{\prime}=1.0776479366167730-0.1141865540435569 \sqrt{-1}
\end{gathered}
$$

We choose these square roots.

When we plug in the above values we find that our matrices satisfy the relations of Lemma 4.5 to high precision. We omit the proof that the matrices satisfy the relations exactly. Most of the proof consists in running the proof of Lemma 5.1 backwards. The only nontrivial part of the proof is taken care by the lucky accident for $\nu$.

\subsection{The Polyhedron}

We are going to build a triangulated complex $Z_{0}^{*}$ in $\boldsymbol{H}^{3}$. First we will specify the vertices of $Z_{0}^{*}$ and then we will specify a triangulation by taking the hyperbolic convex hulls of various 3 -element subsets of the vertices. Figure 4.9 is our constant guide during our construction.

Let $\alpha_{n}$ be the axis of $A_{n}^{*}$. Let $\beta_{n}$ be the axis of $B_{n}^{*}$. We orient $\alpha_{n}$ so that it runs from its endpoint of smaller norm to its endpoint of larger norm. We orient $\beta_{n}$ in the same way. Certain subarcs of $\alpha_{n}$ and $\beta_{n}$ will play the role of the grey arcs in Figure 4.9. 
$A_{n}^{*}$ and $B_{n}^{*}$ commute with the halfturn

$$
C_{n}^{*}=\left[\begin{array}{cc}
0 & \lambda^{n} i \\
\lambda^{-n} i & 0
\end{array}\right]
$$

and so the corresponding axes intersect. Let $\gamma_{n}$ be the axis of $C_{n}^{*}$. Let $\alpha_{n}^{0}=\alpha_{n} \cap \gamma_{n}$ and $\beta_{n}^{0}=\beta_{n} \cap \gamma_{n}$. These symmetry points are the analogues of the centers of the grey arcs in Figure 4.9.

We introduce some notation by way of example. We write $A_{0}^{*}\left(\alpha_{10}\right) \models \alpha_{-3}$ if $A_{0}^{*}\left(\alpha_{10}\right)=\alpha_{-3}$ in such a way that the orientation on $\alpha_{10}$ is carried to the orientation on $\alpha_{-3}$ and the point $A_{0}^{*}\left(\alpha_{10}^{0}\right)$ occurs after the point $\alpha_{-3}^{0}$ in the orientation on $\alpha_{-3}$.

Lemma 5.2 We have the following relations:

$$
\begin{aligned}
& \text { 1. } A_{0}^{*}\left(\alpha_{3}\right) \models \alpha_{-10} \text { and } A_{0}^{*}\left(\alpha_{10}\right) \models \alpha_{-3} \\
& \text { 2. } B_{0}^{*}\left(\alpha_{5}\right) \models \alpha_{-2} \text { and } B_{0}^{*}\left(\alpha_{2}\right) \models \alpha_{-5} \text {. } \\
& \text { 3. } A_{1}^{*}\left(\beta_{6}\right) \models \beta_{-4} \text {. }
\end{aligned}
$$

Proof: We check all the relations numerically, on the endpoints and on the symmetry points. Thus, it suffices to establish the equality of sets in each of the relations. The numerical checks ensure that the orientations are not reversed and that the relevant symmetry points do not come in the wrong order.

Proving that $A_{0}^{*}\left(\alpha_{3}\right)=\alpha_{-10}$ is the same as proving that $A_{0}^{*} A_{3}^{*} A_{0}^{*}=A_{-10}^{*}$. The fifth relation of Lemma 4.5 , rearranged, gives $A_{3}^{*} A_{0}^{*}=B_{-2}^{*} K_{3}^{*}$. Thus $A_{0}^{*} A_{3}^{*} A_{0}^{*}=A_{0}^{*} B_{-2}^{*} K_{3}^{*}$. Reversing and shifting the indices on the fourth relation, we get $A_{0}^{*} B_{-2}^{*}=K_{*}^{-3} A_{-4}^{*}$. So, $A_{0}^{*} A_{3}^{*} A_{0}^{*}=K_{*}^{-3} A_{-4}^{*} K_{*}^{3}=K_{*}^{-10}$. The relation $A_{0}^{*}\left(\alpha_{-10}\right)=\alpha_{3}$ has a similar proof.

Proving that $B_{0}^{*}\left(\alpha_{5}\right)=\alpha_{-2}$ is the same as proving that $B_{0}^{*} A_{5}^{*} B_{0}^{*}=A_{-2}^{*}$. Using the third and fourth relations in Lemma 4.5, and shifting indices appropriately, we have $B_{0}^{*} A_{5}^{*} A_{2}^{*}=A_{-2}^{*} B_{0}^{*} A_{2}^{*}$. Rearranging this equality gives us the desired relation. The relation $B_{0}^{*}\left(\alpha_{2}\right)=\alpha_{-5}$ has a similar proof.

Proving that $A_{1}^{*}\left(\beta_{6}\right)=\beta_{-4}$ is equivalent to proving that $A_{1}^{*} B_{-4}^{*} A_{1}=B_{6}^{*}$. From the relations in Lemma 4.5 , the element $\left(A_{1}^{*} B_{6}^{*} A_{-2}^{*}\right)\left(A_{-2}^{*} A_{1}^{*} B_{-4}^{*}\right)$ is trivial. Simplifying, this gives us the desired relation. 
We remark that all the relations in Lemma 5.2 remain true if we shift the indices by an even number. For instance, $A_{10}^{*}\left(\alpha_{13}\right) \models \alpha_{0}$. Also, we can use the fact that our halfturns are involutions to get other relations. For instance, $A_{-10}\left(\alpha_{-13}\right)=\alpha_{0}$.

Looking at Figure 4.9 we see that each grey arc contains two vertices of the tiling. These grey arcs are parts of the complex lines fixed by the pairing reflections. By analogy we would like to select two points on each halfturn axis which will serve as vertices for our tiling.

If $p$ and $q$ are two points on a geodesic $\gamma$, we write $p \rightarrow_{t} q$ to denote the point $(1-t) p+t q$, computed using the linear structure of $\gamma$ induced by the hyperbolic metric. For instance $p \rightarrow_{1 / 2} q$ is the hyperbolic midpoint of the geodesic segment joining $p$ to $q$. As another example (relevant to the way we compute things)

$$
p \rightarrow_{3 / 4} q=\left(p \rightarrow_{1 / 2} q\right) \rightarrow_{1 / 2} q
$$

We define

$$
\begin{array}{cc}
\alpha_{0}^{-}=\alpha_{0} \rightarrow_{3 / 4} A_{-10}^{*}\left(\alpha_{-13}^{0}\right) & \alpha_{0}^{+}=\alpha_{0} \rightarrow_{3 / 4} A_{10}^{*}\left(\alpha_{13}^{0}\right) \\
\beta_{0}^{-}=\beta_{0}^{0} \rightarrow_{1 / 2} A_{-5}^{*}\left(\beta_{-10}^{0}\right) & \beta_{0}^{+}=\beta_{0}^{0} \rightarrow_{1 / 2} A_{5}^{*}\left(\beta_{10}^{0}\right) \\
\alpha_{1}^{-}=\alpha_{1}^{0} \rightarrow_{1 / 4} A_{-2}^{*}\left(\alpha_{-12}^{0}\right) & \alpha_{1}^{+}=\alpha_{1}^{0} \rightarrow_{1 / 4} A_{4}^{*}\left(\alpha_{14}^{0}\right)
\end{array}
$$

By Lemma 5.2 the points $\alpha_{0}^{-}, \alpha_{0}^{0}, \alpha_{0}^{+}$appear in order on $\alpha_{0}$, in terms of the orientation. By symmetry, these points are evenly spaced in the hyperbolic metric. Indeed, $J_{*}$ swaps $\alpha_{0}^{-}$with $\alpha_{0}^{+}$and fixes $\alpha_{0}^{0}$. See Figure 5.1.

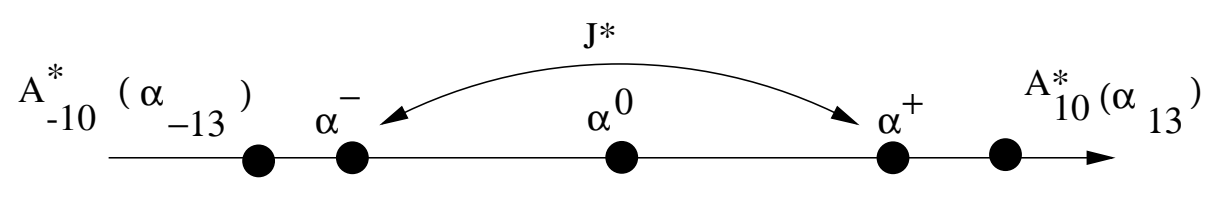

Figure 5.1

The other points have similar properties.

\section{Remarks:}

(i) In defining our points we favored the action of the $A$-halfturns. We could have used some of the $B$-halfturns. The various relations imply that we would get the same result. We will see this below in a key case.

(ii) The choice of $3 / 4$ and $1 / 4$ is somewhat arbitrary. We could take any $t$ 
and $(1-t)$ and get the same symmetries. We choose $t=3 / 4$ because it makes the picture look nice and because the relevant point is easy to compute, by taking successive midpoints, as in Equation 66.

Now we are ready to define our tiles. We define the join operation in $\boldsymbol{H}^{3}$ using geodesic segments. As in $\S 4$ the notation $[\ldots] \square$ means the geodesic polygon whose vertices are the points listed. We define

$$
Z_{0}^{*}=\bigcup A^{*}(n) \cup B^{*}(2 m),
$$

where

$$
\begin{array}{r}
A_{*}(0)=\alpha_{0}^{0} \bowtie\left[\alpha_{0}^{-}, \alpha_{-10}^{+}, \beta_{-8}^{+}, \alpha_{-3}^{+}, \alpha_{0}^{+}, \alpha_{10}^{-}, \beta_{8}^{-}, \alpha_{3}^{-}\right] \square . \\
B^{*}(0)=\beta_{0}^{0} \bowtie\left[\alpha_{-5}^{+}, \beta_{0}^{-}, \alpha_{2}^{-}, \alpha_{5}^{-}, \beta_{0}^{+}, \alpha_{-2}^{+}\right] \square . \\
A^{*}(1)=\alpha_{1}^{0} \bowtie\left[\alpha_{1}^{-}, \beta_{6}^{-}, \alpha_{1}^{+}, \beta_{-4}^{+}\right] \square \\
A^{*}(n+2 k)=K_{*}^{n}\left(A^{*}(k)\right) ; \quad B^{*}(2 m)=K_{*}^{-}\left(B^{*}(0)\right) .
\end{array}
$$

In making our definitions, we looked at each relevant tile in Figure 4.9 and listed out the labels of the grey curves which attach to its vertices, using - and + depending on whether or not the attaching point is at the top or the bottom of the grey curve. Figure 5.2 shows a schematic picture.
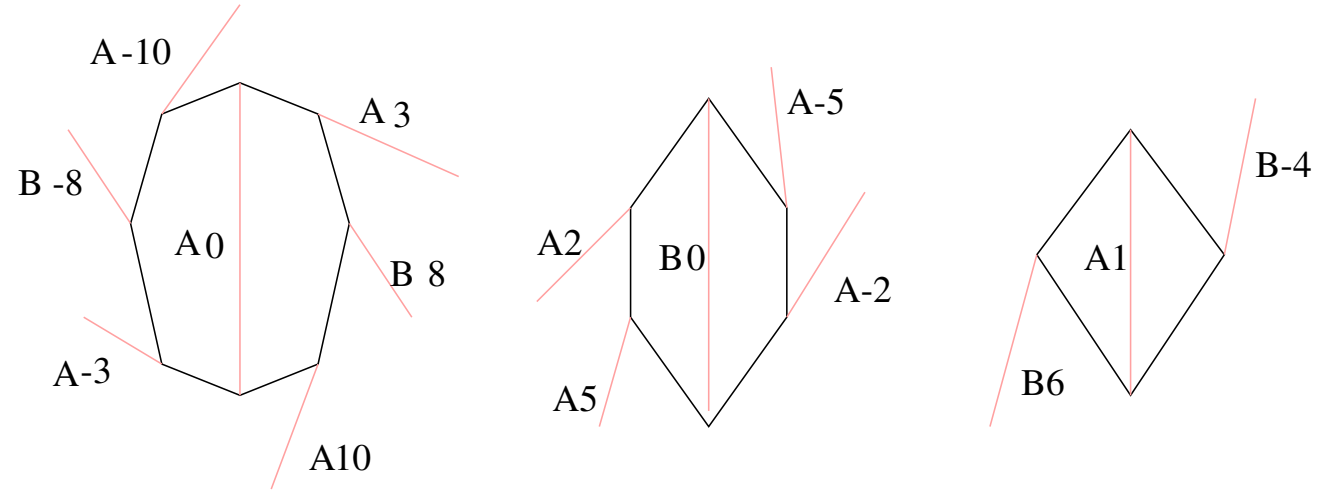

Figure 5.2

Lemma 5.3 Equation 45 holds (with the stars added) for our tiles. 
Proof: Our constructions are symmetric with respect to $J_{*}$ and $K_{*}$. Using this symmetry, and the fact that the halfturns are involutions, it suffices to prove that

$$
A_{1}^{*}\left(\beta_{6}^{-}\right)=\beta_{-4}^{+} ; \quad A_{0}^{*}\left(\alpha_{3}^{-}\right)=\alpha_{-10}^{+} ; \quad A_{0}^{*}\left(\beta_{8}^{-}\right)=\beta_{-8}^{+} ; \quad B_{2}^{*}\left(\alpha_{7}^{-}\right)=\alpha_{0}^{+} .
$$

The first two relations follow from Lemma 5.2 and from the definitions.

For the third relation, we first establish an auxilliary identity. We know that $B_{-2}^{*} A_{3}^{*} A_{0}^{*}=K^{3}$. This is the fourth relation in Lemma 4.5. Thus $A_{3}^{*} A_{0}^{*}=B_{-2} K^{3}$. Thus $K^{-3} A_{3}^{*} A_{0}^{*}=K^{-3} B_{-2} K^{3}=B_{-8}$. This last relation is equivalent to $A_{3}^{*} A_{0}^{*} B_{-8}^{*}=K^{3}$. Therefore,

$$
\beta_{-2}^{+}=K_{*}^{3}\left(\beta_{-8}^{+}\right)=A_{3}^{*} A_{0}^{*} B_{-8}^{*}\left(\beta_{-8}^{+}\right)=A_{3}^{*} A_{0}^{*}\left(\beta_{-8}^{+}\right) .
$$

It follows from our definitions that $A_{3}^{*}\left(\beta_{-2}^{+}\right)=\beta_{8}^{-}$. (This is just the first relation in Equation 69, with the indices shifted by 4.) Combining this with Equation 70 we get the third relation in Equation 69.

A rearranged version of the second relation of Equation 69 gives the relation $A_{4}^{*}\left(\alpha_{-6}^{+}\right)=\alpha_{7}^{-}$. Also, $B_{2}^{*} A_{7}^{*} A_{4}^{*}=K^{3}$. Thus

$$
\alpha_{0}^{+}=K^{3}\left(\alpha_{-6}^{+}\right)=B_{2}^{*} A_{7}^{*} A_{4}^{*}\left(\alpha_{-6}^{+}\right)=B_{2}^{*} A_{7}^{*}\left(\alpha_{7}^{-}\right)=B_{2}^{*}\left(\alpha_{7}^{-}\right) .
$$

The last equality comes from the fact that $\alpha_{7}^{-}$is fixed by $A_{7}^{*}$.

By construction $Z_{0}^{*}$ is an immersed infinite cylinder, invariant under the elements $K_{*}$ and $J_{*}$. We will draw pictures of $Z_{0}^{*}$ and argue that it is in fact an embedded cylinder which links the axis of $K_{*}$. The logic behind our pictorial proof runs as follows: We think of $Z_{0}^{*}$ as the image of a combinatorially defined cylinder $\widetilde{Z}_{0}$ under a map $\phi: \widetilde{Z}_{0} \rightarrow Z_{0}^{*}$. It suffices to prove that the composition $\pi \circ \phi$ maps $\widetilde{Z}_{0}$ homeomorphically onto the punctured plane. This is true iff $\pi\left(Z_{0}^{*}\right)$ is a triangulation of the punctured plane. (Recall that $\pi$ maps geodesics to straight line segments.) By looking at the pictures we can see that this is the case. We omit the straightforward numerical analysis which shows that our pictures are accurate enough. In $\S 10.3$ we will sketch our method for drawing these pictures.

Figures 5.3, 5.4 and 5.5 respectively show $A^{*}(0), B^{*}(0)$ and $A^{*}(1)$. In all the figures, the darkest line is the projection of halfturn axis. 


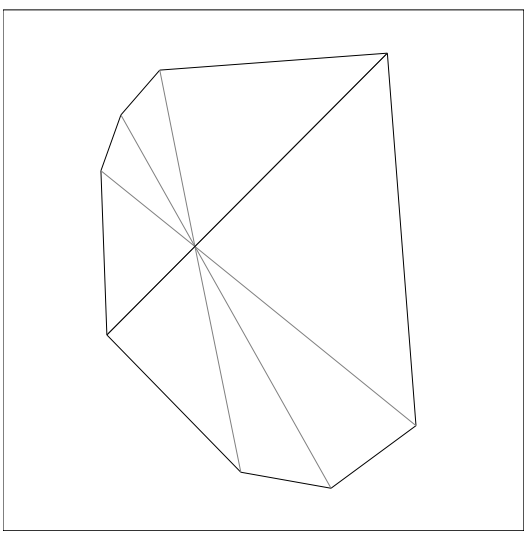

Figure 5.3

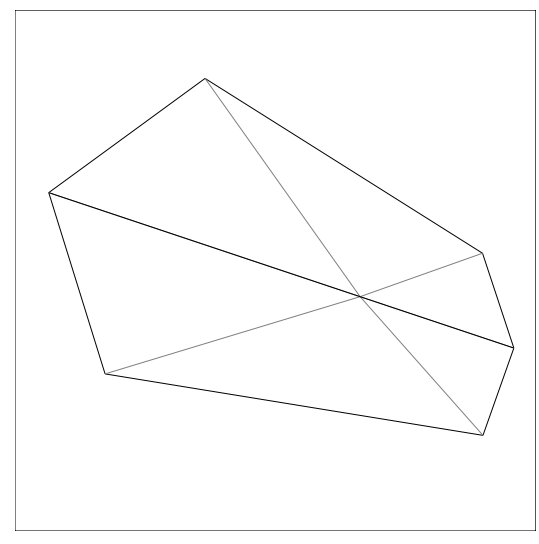

Figure 5.4

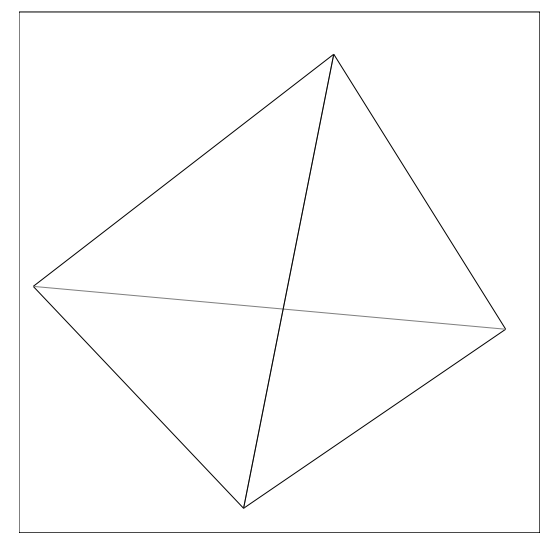

Figure 5.5 
Figure 5.6 shows the whole tiling. We have erased most of the lines which lie inside the tile, leaving only the lines of (hyperbolic) bilateral symmetry, which are drawn in grey.

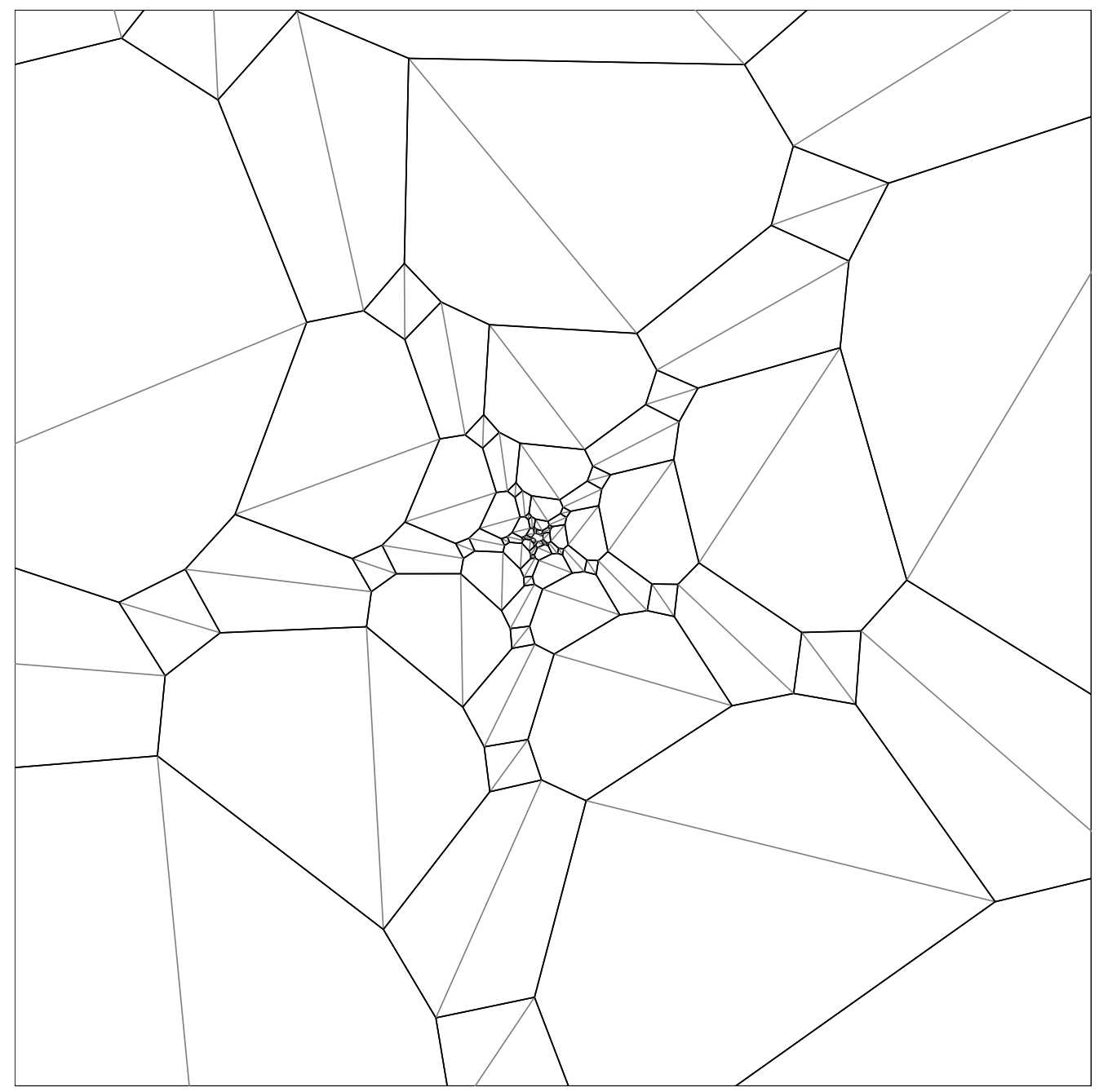

Figure 5.6

Looking at Figure 5.6 we can see that $Z_{0}^{*}$ is an embedded cyclinder which links the axis of $K_{*}$. Let $\Delta_{0}^{*}$ be the component of $\boldsymbol{H}^{3}-Z_{0}^{*}$ which contains this axis. Then $\Delta_{0}^{*}$ is an infinite open solid cylinder with boundary $Z_{0}^{*}$. The quotient $\left(\Delta_{0}^{*} \cup Z_{0}^{*}\right) / K_{*}^{3}$ is clearly compact. 


\subsection{The Transcription}

Each face of $Z_{0}^{*}$ is paired to itself by one of the halfturn pairings. The grey lines are the projections of the axes of these halfturn pairings. Being equivariant under the action of $K_{*}$, the action of the halfturn pairings descents to the quotient $Q_{0}^{*}=\left(\Delta_{0}^{*} \cup Z_{0}^{*}\right) / K_{*}^{3}$, which is a solid torus with polyhedral boundary. Let $Q_{0}^{*} / \sim$ be the identification space obtained by folding each tile in half, via the relevant halfturn pairing. The relations satisfied by the halfturn pairings, given in Lemma 4.5, imply that the edges of $Q_{0}^{*}$ are identified in groups of 3, and that the dihedral angle around each edge (away from a vertex) is $2 \pi$. The dihedral angle around the axes of the halfturn pairings is $\pi$. Everywhere else, the quotient is a manifold. In short, $Q_{0}^{*}$ is a compact hyperbolic orbifold.

Let $\Gamma^{*}(4,7)$ be the group generated by the halfturn pairings. The fact that $Q_{0}^{*}$ is a compact orbifold implies that $\Gamma^{*}(4,7)$ is a co-compact lattice in Isom $\left(\boldsymbol{H}^{3}\right)$ and that every relation in $\Gamma^{*}(4,7)$ is a consequence of the ones in Lemma 4.5. These facts are typically established in connection with Poincarè's theorem on fundamental polyhedra $[\mathbf{B}]$. In particular, there is a homomorphism $h: \Gamma^{*}(4,7) \rightarrow \Gamma(4,7)$ which extends the obvious map sending $A_{n}^{*}$ to $A_{n}$ and $B_{n}^{*}$ to $B_{n}$.

Lemma $5.4 h$ is surjective.

Proof: Looking at the chart in $\S 4.8$ we see that $I_{1}, I_{2} I_{1} I_{2}$ and $I_{2} I_{3} I_{2}$ are all in the image of $h$. Note that $I_{2}=\left(I_{2} I_{3} I_{2}\right)\left(I_{2} I_{1} I_{2}\right)\left(I_{2} I_{1} I_{3}\right)$. Hence $I_{2}$ is in the image of $h$. By symmetry, so is $I_{3}$.

Let $\left[Z_{0}\right]$ be as in $\S 4$. Recall from $\S 4.1$ that $\left[E_{0} K\right]$ and $\left[E_{\infty} K\right]$ are the two fixed points of $I_{2} I_{1} I_{3}$. In $\S 6-10$ we will prove that $\left[Z_{0}\right] \cup\left[E_{0} K\right] \cup\left[E_{\infty} K\right]$ is a tamely embedded 2 -sphere and that one of the complementary components $\Delta_{0}$ is such that the $\Gamma(4,7)$-orbit of $\Delta_{0} \cup\left[Z_{0}\right]$ tiles the domain of discontinuity $\Omega(4,7)$. Moreover, we will show that $\Omega(4,7) / \Gamma(4,7)=Q_{0} / \sim$, a space defined just as above, but with the stars left off. Modulo these details, there is a homeomorphism $h^{\prime}: \Delta_{0}^{*} \cup Z_{0}^{*} \rightarrow \Delta_{0} \cup\left[Z_{0}\right]$ which intertwines the halfturn pairings of this chapter with the reflection pairings of $\S 4$.

It follows formally from all of this that $h^{\prime}$ extends to a universal covering map $h^{\prime \prime}: \boldsymbol{H}^{3} \rightarrow \Omega(4,7)$ which is compatible with the homomorphism $h$. Thus $h^{\prime \prime}$ (or $h^{\prime}$ ) induces an orbifold homeomorphism from $Q_{0}^{*} / \sim$ to $Q_{0} / \sim$. 
This puts the hyperbolic orbifold structure on $\Omega(4,7) / \Gamma(4,7)$.

\section{Remarks:}

(i) $\boldsymbol{H}^{3}$ contains a network of geodesics, the axes of halfturns which are $\Gamma^{*}(4,7)$-conjugate to the halfturn pairings. $h^{\prime \prime}$ maps each geodesic in this network to an arc of a $\boldsymbol{C}$-circle. The endpoints of this arc are contained in the limit set $\Lambda(4,7)$ and the rest of the arc is contained in $\Omega(4,7)$. There is a second network of geodesics in $\boldsymbol{H}^{3}$, corresponding to elements which are $\Gamma^{*}(4,7)$-conjugate to the axes of the $C_{n}^{*}$ halfturns. $h^{\prime}$ can be chosen so as to respect the dihedral symmetry of the domain and range, meaning that $h^{\prime}$ conjugates the $C_{n}^{*}$ halfturns to the antiholomorphic symmetries of $\left[Z_{0}\right]$. Thus $h^{\prime \prime}$ maps each geodesic in the second network to an $\boldsymbol{R}$-circle, necessarily contained in $\Omega(4,7)$. The model for the map is the univeral covering from the line to the circle. Considering the two networks at the same time, it seems to us that there is a kind of fibered quality to the covering $h^{\prime \prime}: \boldsymbol{H}^{3} \rightarrow \Omega(4,7)$. Geodesics in the first network are never wrapped up and geodesics in the second network are always wrapped up.

(ii) In $[\mathbf{K}]$ one can read how the usual covering of the modular surface by $\boldsymbol{H}^{2}$ is given as a ratio of modular forms. We wonder if there is a similar description for a covering map $\boldsymbol{H}^{3} \rightarrow \Omega(4,7)$ which is isotopic to $h^{\prime \prime}$.

\subsection{Topology of the Quotient}

Let $N_{\epsilon}$ be the $\epsilon$-neighborhood of the axis of $K_{*}$ in $\boldsymbol{H}^{3}$. If $\epsilon$ is small then $N_{\epsilon}$ is contained in the interior of $\Delta_{0}^{*}$. Choose such an $\epsilon$ and let $Q_{01}^{*}=N_{\epsilon} / K^{3}$. Let $Q_{02}^{*}$ be the closure of $Q_{0}^{*}-Q_{01}^{*}$. We have $Q_{0}^{*}=Q_{01}^{*} \cup Q_{02}^{*}$. The common boundary is a torus.

$Q_{02}^{*}$ is product of a torus with an interval. The outer boundary component of $Q_{02}^{*}$ has the tiling $Z_{0}^{*} / K_{*}^{3}$ on it. We can foliate each tile of this tiling by line segments, running transverse to the line of bilateral symmetry of the tile, in a way which respects the bilateral symmetry. Figure 5.7 shows a schematic picture. (The tiling in Figure 5.7 is locally equivalent to the ones in Figures 4.1, 4.9 and 5.6.) The dark arrow in Figure 5.7 indicates the action of $K_{*}^{3}$. 


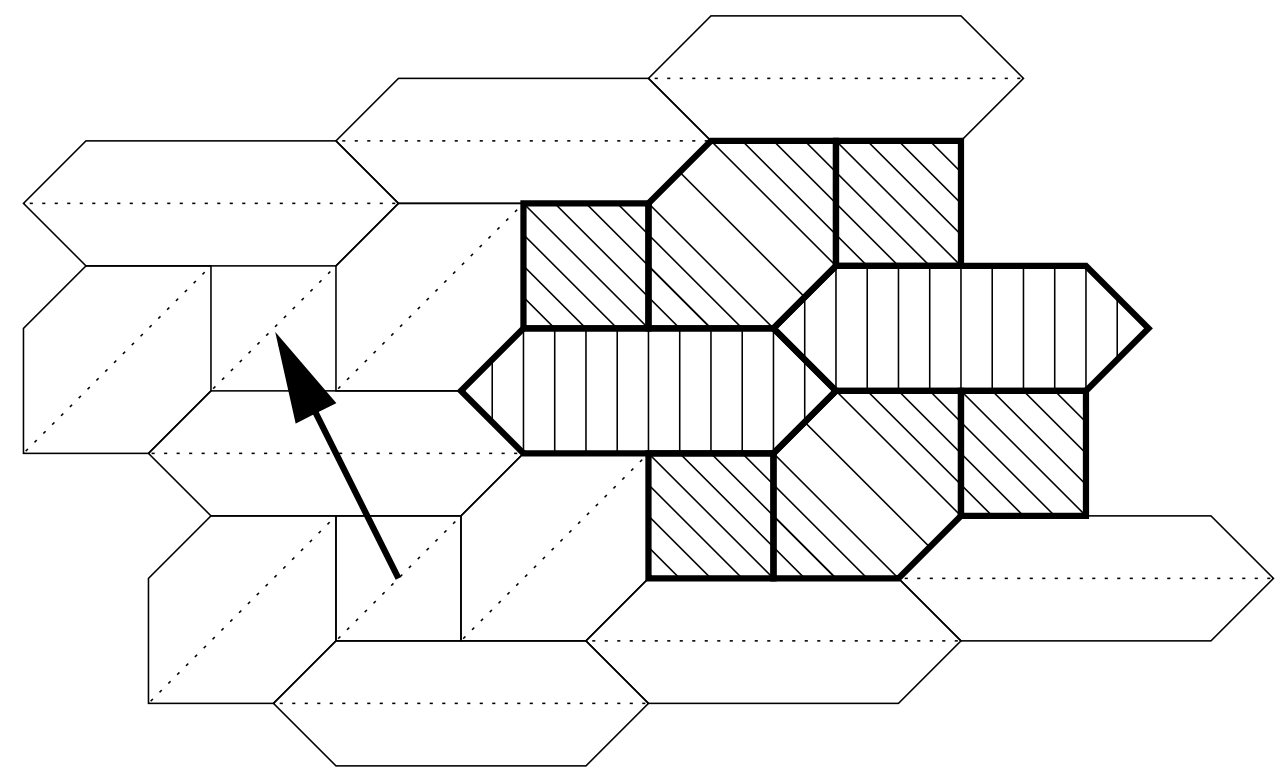

Figure 5.7

The foliations on each tile piece together to give a circle foliation of the outer boundary component of $Q_{02}^{*}$. Using the product structure on $Q_{02}^{*}$ we obtain an annulus foliation of $Q_{02}^{*}$. Here is the key observation: By construction, the identifications on $Q_{02}^{*}$ induced by $\sim$ respect this foliation.

Let $T_{1}$ be a torus of revolution in $\boldsymbol{R}^{3}$. Let $T_{2}$ be a thinner torus of revolution contained in the interior of $T_{1}$. Note that $\partial Q_{01}^{*}$ inherits a circle foliation from the circle foliation on the inner boundary of $Q_{02}^{*}$. We identify $Q_{01}^{*}$, a solid torus, with $\left(\boldsymbol{R}^{3} \cup \infty\right)-T_{1}$ in such a way that each circle in the foliation on $\partial Q_{01}^{*}$ is trivial in the first homology group $H_{1}\left(T_{1}\right)$. We idenfity $Q_{02}^{*}$ with $T_{1}-T_{2}$. This region is obtained by revolving an annulus about the $z$-axis, and the obvious foliation by annuli is identified with our foliation of $Q_{02}^{*}$.

The first half of Figure 5.8 shows a generic leaf of our annulus foliation. The three white points are the intersections of the boundary of the annulus with the edges of the tiles. The three black points are the intersections of the same boundary with the lines of symmetry of the tiles. The curved arrows indicate the identifications to be made from the folding. The second half of Figure 5.8 shows what happens when the identifications are made. The result is simply a disk with 3 cone points labelled by $\boldsymbol{Z} / 2$. 

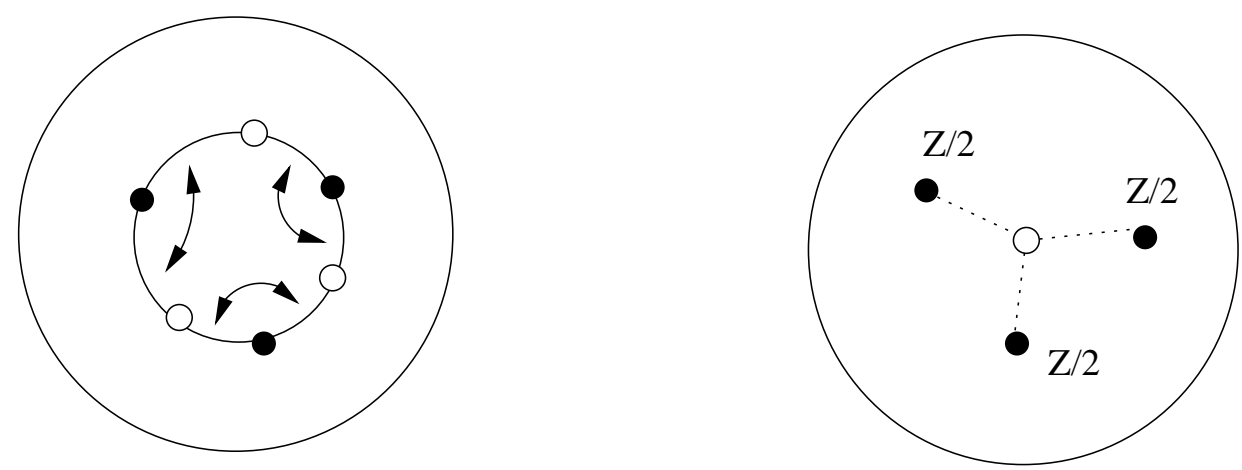

Figure 5.8

There are 9 exceptional fibers in our annulus foliation; these are the ones which intersect vertices of the tiling. It is easy to see that the exceptional fibers have the same quotients, topologically speaking, as the generic ones. Hence $Q_{02}^{*} / \sim$ is a solid torus with a 3 -strand braid of $\boldsymbol{Z} / 2$ cone singularities. We identify this solid torus with $T_{1}$. All in all, $Q_{0}^{*} / \sim$ has underlying space $S^{3}$, and singularity locus a 3 -strand braid equipped with a $\boldsymbol{Z} / 2$ cone singularity structure.

We can draw the braid. The key to doing this is to make the right hand side of Figure 5.8 vary canonically, and hence continuously, with the left hand side. We parametrize the inner boundary of the annulus on the left by $\theta \in[0,2 \pi]$. To each black point $p$ on the left we can assign the pair $(\theta(p), d(p))$. Here $\theta(p)$ is the coordinate of $p$ on the circle and $d(p)$ is the distance between the white points which flank $p$. On the right, we place a black point $p^{\prime}$ at the point

$$
\frac{d(p)}{10} \exp (i \theta(p))
$$

The semi-arbitrary factor of $1 / 10$ is present to keep $p^{\prime}$ inside the disk at right.

In an exceptional fiber, one of the $d$-values is zero. Note that $p^{\prime}=0$ when $d(p)=0$. This phenomenon allows our placement to vary continuously even when we pass through a singular fiber. What happens is that one of the cone points passes through the origin and then continues on its way.

When we perform this construction for each of the fibers (or, more practically, for 1000 sample fibers) we obtain three curves which run through $T_{2}$. Projecting these curves to a plane we get the braid shown in Figure 5.9. 


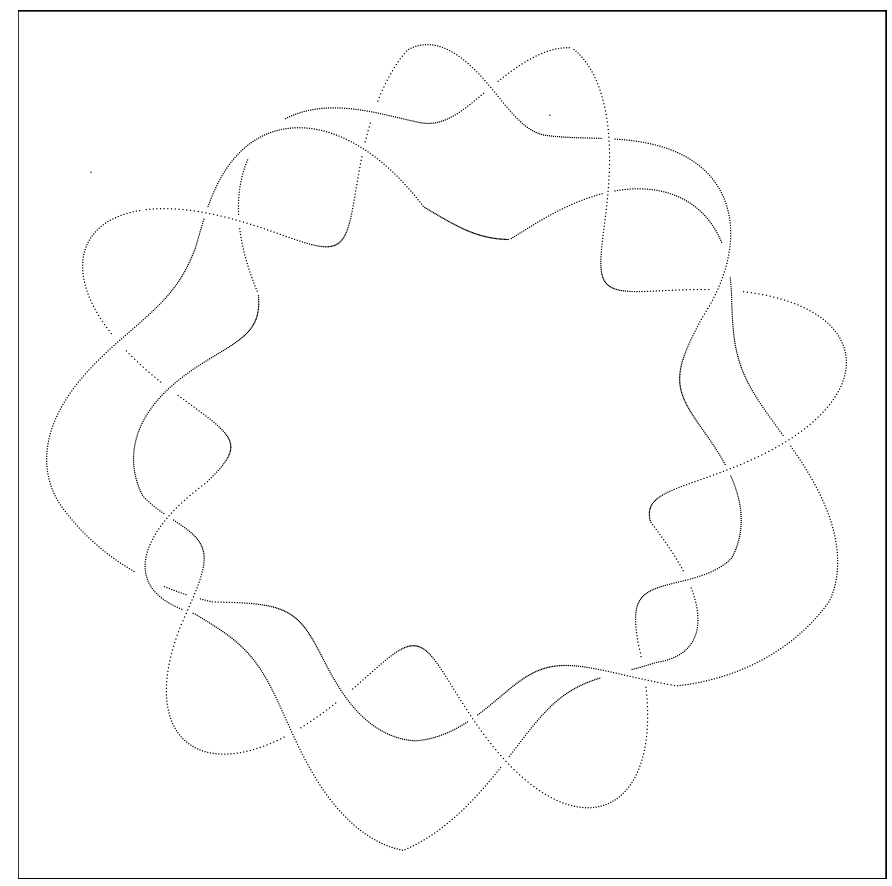

Figure 5.9

The standard generators of the braid group are given by

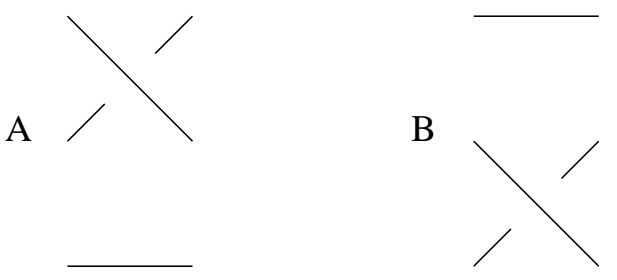

Figure 5.10

We can identify $A$ (or $A^{-1}$ ) with the crossings that occur near the outside of Figure 5.9, and $B$ (or $B^{-1}$ ) with the crossings that occur near the inside. For instance, the crossing closest to the top edge of Figure 5.9 counts as an $A$. In this way, the braid in Figure 5.9 can be interpreted as a word in positive powers of $A$ and $B$.

This braid in Figure 5.9 is rather too complicated for our taste. When we perform 5 Dehn twists of $T_{1}$ (respecting the annulus foliation) we get the braid shown in Figure 5.11. 


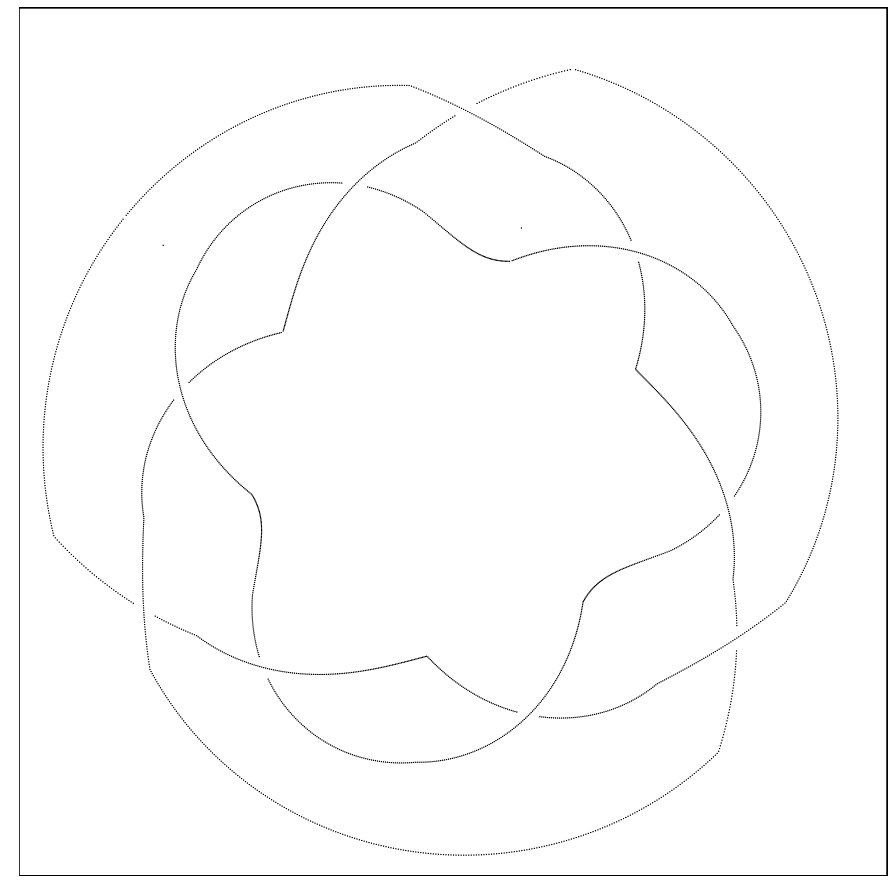

Figure 5.11

This braid has representation $\left(A B^{-2}\right)^{3}$. A single Dehn twist introduces the element $(A B)^{-3}$. Thus, $(A B)^{-15}$ times the braid in Figure 5.9 is the braid in Figure 5.11. Hence, the braid in Figure 5.9 is equivalent to $(A B)^{15}\left(A B^{-2}\right)^{3}$, the braid mentioned in connection with Theorem 1.1. 


\section{The View from the Inside}

\subsection{Overview}

We defined $Z$ in Equation 29 of $\S 4$. Figure 6.0, which is a compendium of pictures from $\S 4$, shows the building blocks of $Z$. We have left off the word notation, as we will not need it below.
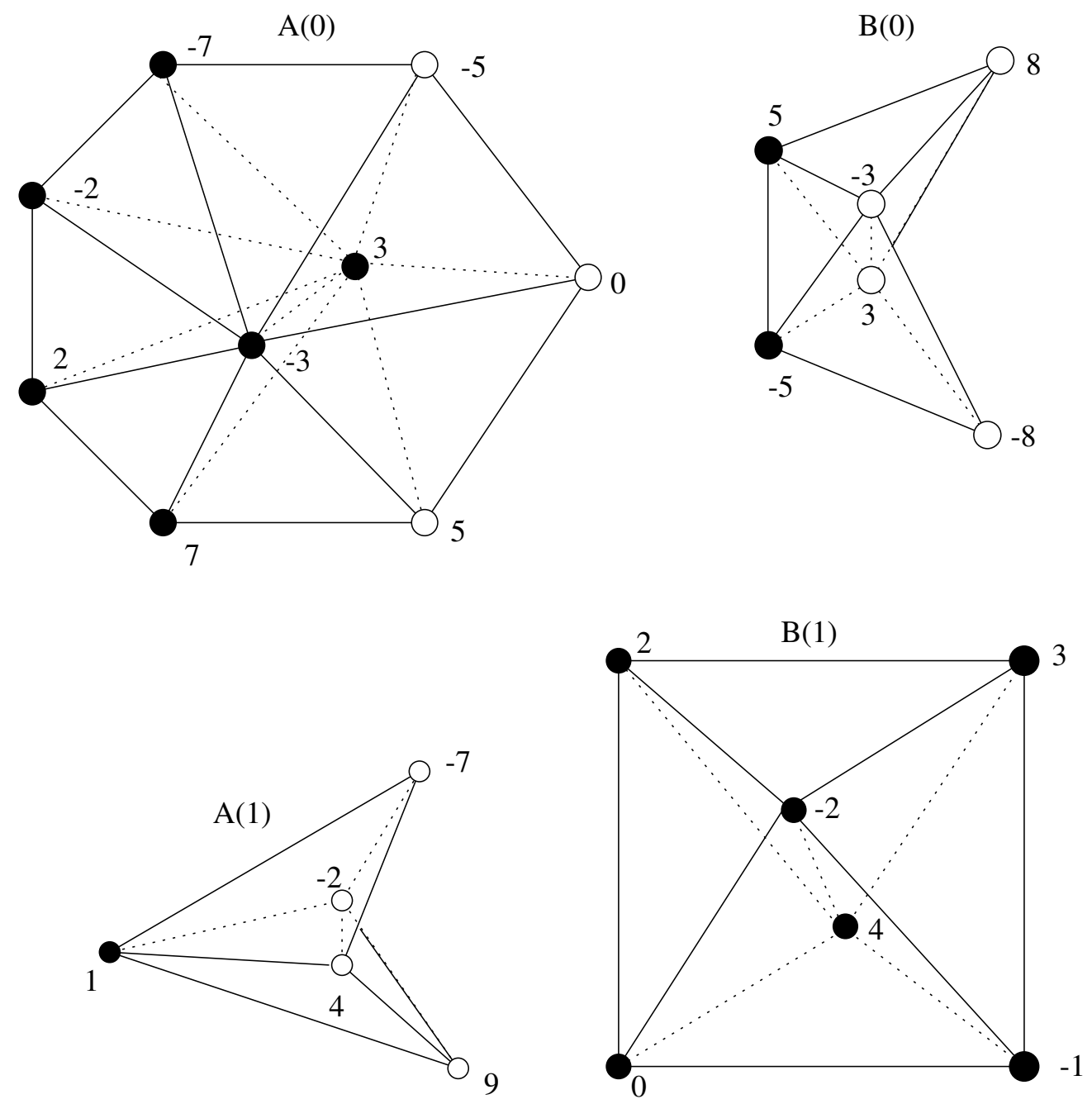

Figure 6.0

We also worked out the symmetries of these pieces. These symmetries are given in Lemmas 4.1-4.4. In this chapter we will analyze how these pieces fit 
together. That is, we will analyze the combinatorics and topology of $Z$ and $[Z]$. During our analysis we will make 3 assuumptions:

Combinatorial Assumption: We assume that the intersection of any pair of tetrahedra in $Z$ is exactly the convex hull of the set of vertices common to both. This assumption allows us to piece $Z$ together simply by looking at the vertices.

Homeomorphism Assumption: We assume that the projectivization map $\Theta: Z \rightarrow[Z]$ is a homeomorphism.

Purity Assumption: We say that a pure simplex in $Z$ is one whose vertices all have the same type, positive or negative. For example $\bullet$ $0 \bowtie$ is a pure negative edge. We make the assumption that all the vectors in a pure simplex have the same type. This assumption allows to predict the types of all the vectors in a simplex just by looking at the dots in the Figure 6.0.

In this chapter we will not dwell on the validity of the assumptions. (It turns out that the second one is flawed, and we will patch this up in §8.) The point is that we want to analyze everything combinatorially first.

We define $A(2 n, \pm)=K^{n} A(0, \pm)$, where

$$
\begin{array}{r}
A(0,-)=[\bullet 3 \bullet(-3)] \bowtie[\bullet(-7) \bullet(-2) \bullet 2 \bullet 7] ; \\
A(0,+)=[\bullet 3 \bullet(-3)] \bowtie[\bullet 7 \circ 5 \circ 0 \circ(-5) \bullet(-7)]
\end{array}
$$

Note that $A(2 n)=A(2 n,+) \cup A(2 n,-)$. We split this tile apart for the purposes of visualization only. We will build $Z$ in three layers, writing $Z=Z_{1} \cup Z_{2} \cup Z_{3}$, where

$$
Z_{1}=\bigcup_{m=-\infty}^{\infty} B(2 m+1) ; \quad Z_{2}=\bigcup_{m=-\infty}^{\infty} A(2 m,-) ; \quad Z_{3}=Z-\left(Z_{1} \cup Z_{2}\right)
$$

Looking at the vertices in Figure 6.0 we see that $Z_{1} \cup Z_{2} \subset N_{-}$. All the pictures from $\S 4$ come from $Z_{3}$. Informally speaking, $Z_{2}$ fits around $Z_{1}$ like an infinite sheath fits around an infinite sword. $Z_{3}$ fits around $Z_{1} \cup Z_{2}$ in the same way. Figure 6.1 shows a schematic picture, both from the side and from the front. 

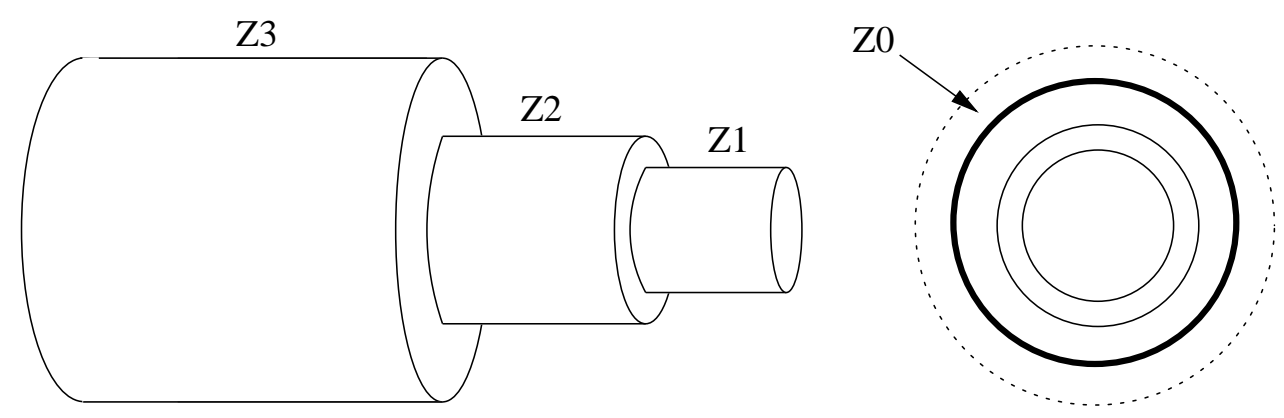

Figure 6.1

It is useful to make a small modification of $Z$. Henceforth we delete from $Z$ all the pure positive simplices. The maximum dimension of such a simplex is 2 . Given the purity assumption, this causes no harm: We only care about $Z \cap\left(N_{-} \cup N_{0}\right)$. The reason that we delete the positive simplices is that it simplifies the topology of $Z$. It will allow us to write $Z_{3}=Z_{0} \times(-1,1)$. Here $Z_{0}$ is the set of null vectors in $Z$.

Let $D$ be the open disk of radius 2 centered at the origin in the plane. Let $S^{1} \subset D$ be the unit circle. One main goal of this chapter is to prove

Lemma 6.1 (Combinatorial Lemma) The pair $\left([Z],\left[Z_{0}\right]\right)$ is homeomorphic to the pair $\left(D, S^{1}\right) \times \boldsymbol{R}$.

Following the proof of the Combinatorial Lemma we will analyze the action of the reflection pairings on the triangles of $[Z]$. For reference these reflection pairings are shown in the chart in $\S 4.9$.

\subsection{Building $Z$}

Looking at Figure 6.0, and recalling Equation 37, we see that $B(1)$ and $B(3)$ share the vertices $\bullet 0, \bullet 2$ and $\bullet 4$. Likewise $B(1)$ and $B(5)$ share the vertices $\bullet 2 \bullet 3$ and $\bullet 4$. Finally, $B(1)$ and $B(2 m+1)$ have no vertices in common if $|m| \geq 3$. Hence $B(2 m+1) \cap B(2 m+3)$ is a triangular face, $B(2 m+1) \cap B(2 m+5)$ is a triangular face, and all other intersections are empty.

$Z_{1}$ is built from an infinite supply of "octahedra" as follows: First, glue the octahedron corresponding to $B(2 m+1)$ to the one corresponding to $B(2 m+3)$ along the common face. The result is a solid cylinder. After this step, glue the face on the octahedron corresponding to $B(2 m+1)$ to the 
corresponding face on $B(5 m+1)$. These two faces share a common edge, one the gluing merely folds the faces together across this edge, like a hinge closing. It is not hard to see (especially if one builds a model) that the resulting space is homeomorphic to a solid cylinder.

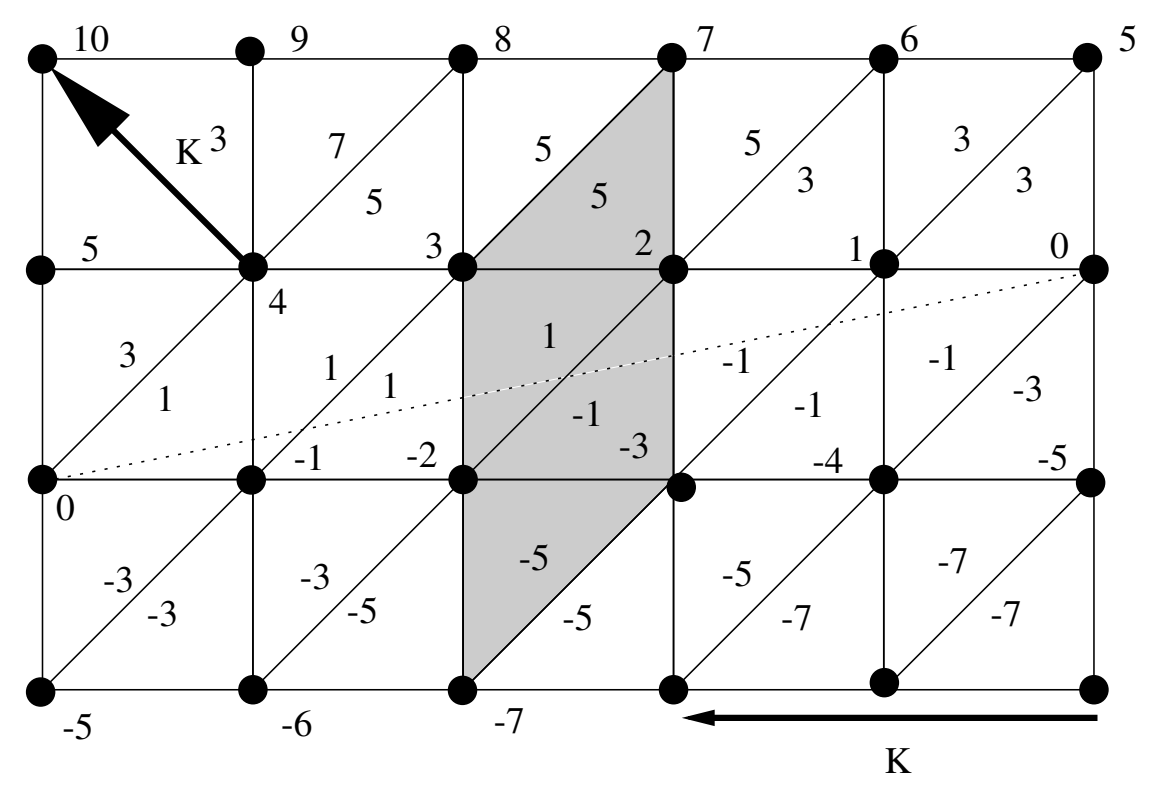

Figure 6.2

$\partial Z_{1}$ is tiled by triangles. Figure 6.2 shows the lift to $\boldsymbol{R}^{2}$ of this tiling. The dotted line segment connects a point to its image under the generator of the deck group. We have labelled each triangle by the integer $k$ if that triangle is a face of $B(k)$. We have also indicated the action of $K$ and $K^{3}$. The action of $J$, defined in Equation 19, has the effect of rotating the figure by 180 degrees about its center. The circular dot labelled $k$ represents the point $\bullet k$.

Before we start building $Z_{2}$ we want to bring up a different analogy, which may help with the visualization. Suppose we are construction workers and we have just built an infinite cylindrical tower. We would like to add a layer of bricks around the outside of the tower, so as to thicken it. The shaded parallelogram in Figure 6.2 indicates the location of one of the attaching-spots. (The whole surface of $Z_{1}$ is tiled by translates of this parallelogram.) The brick we attach to the shaded parallelogram is $A(0,-)$. The only misleading part of our analogy is that $Z_{1}$ is not contained in the interior of $Z_{1} \cup Z_{2}$. The boundaries $\partial Z_{1}$ and $\partial\left(Z_{1} \cup Z_{2}\right)$ share all the vertices $\bullet(2 m+1)$, and the 
edges joining these vertices. Thus, our bricks are rather peculiar; the top and bottom faces are not completely separated by the sides, but rather taper down towards each other and meet along a pair of opposite edges. In Figure 6.3 these opposite edges are $\bullet 7 \bowtie \bullet$ and $\bullet(-7) \bowtie \bullet(-3)$.

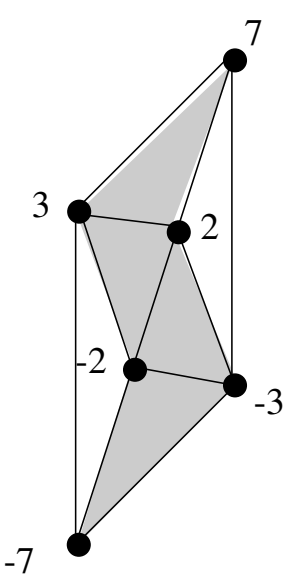

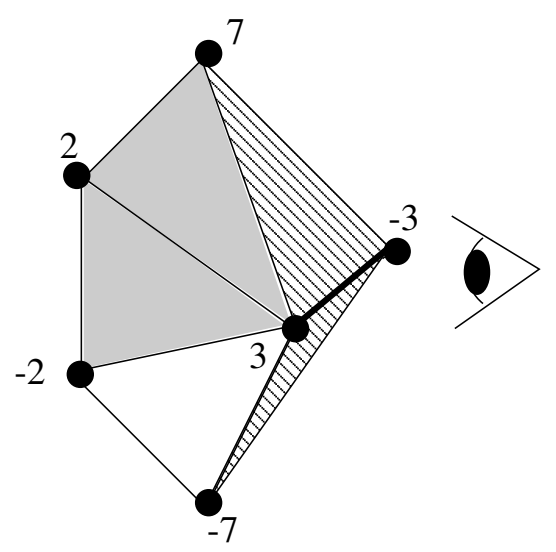

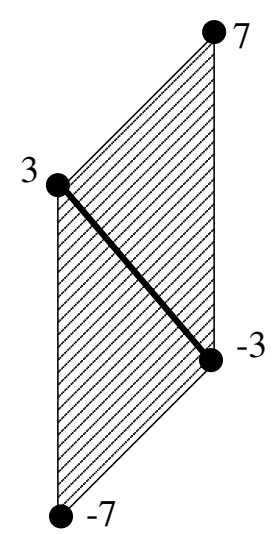

Figure 6.3

The middle of Figure 6.3 shows a side view of $A(0,-)$. The right hand side of the figure shows the front of $A(0,-)$, from the point of view of the eye. We think of this as the top of $A(0,-1)$. The left hand side shows a (slightly distorted) view of what we think of as the bottom of $A(0,-)$. Note that the shaded part on the left is just a distorted copy of the shaded parallelogram in Figure 6.2. Thus, the bottom of $A(0,-)$ attaches onto $Z_{1}$. The unshaded part on the left is what we call the sides of $A(0,-)$.

Anyone who lays bricks around a tower would know that it is not enough to attach the bricks to the tower itself. The bricks have to be attached to each other, on their sides. Looking at the labelling of $A$ shown in Figure 6.2, we can see that $A(2 m,-)$ and $A(2 n,-)$ share a common triangular side iff $|m-n|=2$. For instance, $A(0,-)$ and $A(4,-)$ share the vertices $\bullet 2, \bullet(-3)$ and $\bullet 7$. These relations are what glue the sides of the bricks together.

The pieces $A(2 m,-)$ fit around $Z_{1}$ to make a fatter solid cylinder. Figure 6.4 shows $\partial\left(Z_{1} \cup Z_{2}\right)$ in the same way that Figure 6.2 shows $\partial Z_{1}$. The vertices are labelled as in Figure 6.2. 


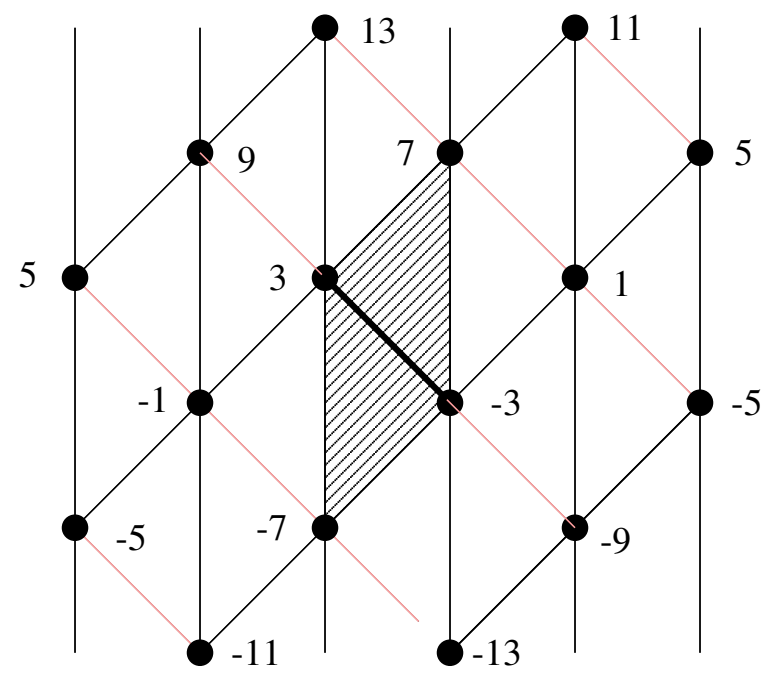

Figure 6.4

Before we put the third layer together, we analyze an example. One of the tetrahedra in $Z_{3}$ has vertices $\bullet(-3), \bullet 3, \circ 0, \circ 5$. Call this tetrahedron $T$. The maximal positive simplex of $T$ is $T_{+}=\circ 0 \bowtie \circ 5$. We have already deleted this edge from $Z$. Thus $T$ does not contain this edge. The maximal negative simplex is $T_{-}=\bullet(-3) \bowtie \bullet 3$. But this edge lies in $\partial Z_{2}$. It is the bold edge shown in Figure 6.4. In short, $T$ does not contain its pure simplices. We think of $T$ as the open join of $T_{+}$and $T_{-}$. That is, $T$ is the union of the open line segments which connect $T_{-}$to $T_{+}$. Under the purity assumption, every such segment joins a point in $N_{+}$to a point in $N_{-}$.

Lemma 6.2 Let $l$ be a line segment which joins a point in $N_{-}$to a point in $N_{+}$. Then the projectivization $[l]$ intersects $S^{3}$ once, transversally. Hence $l$ intersects $N_{0}$ once.

Proof: The projectivization $[l]$ is a circular arc, one of whose endpoints lies outside $S^{3}$ and one of whose endpoints lies inside $S^{3}$. Such a circular arc intersects $S^{3}$ exactly once, transversely. This intersection point corresponds to $l \cap N_{0}$.

Let $T_{0}=T \cap N_{0}$. It follows from Lemma 6.2 that

$$
T=T_{0} \times(-1,1)
$$


By "equal", we mean homeomorphic.

Every tetrahedron in $Z_{3}$ is the open join of its maximal positive and negative sub-simplices. Equation 75 holds for every tetrahedron in $Z_{3}$. Therefore

$$
Z_{3}=Z_{0} \times(-1,1)
$$

Given a tetrahedron $T$, the cross section $T_{0}$ depends on the way the vertices are partitioned. If $T$ has 2 positive vertices and 2 negative vertices then $T_{0}$ is a quadrilateral. If $T$ has 3 vertices of one type and 1 vertex of the other type then $T_{0}$ is a triangle. Compare the heuristic comments at the end of $\S 4.6$.

The inner boundary of $Z_{3}$ is $Z_{0} \times\{-1\}$, which we can identify with $\partial\left(Z_{1} \cup Z_{2}\right)$. Thus $Z$ is obtained from $Z_{1} \cup Z_{2}$ simply by adding on a "collar" neighborhood. Thus $Z$ is homeomorphic to $D \times \boldsymbol{R}$. We can identify $Z_{0}$ with $Z_{0} \times\{0\} \subset Z_{3}$, a set which is homeomorphic to $S^{1} \times \boldsymbol{R}$. This establishes the Combinatorial Lemma.

As one more piece of information we show that (under our three assumptions) $[Z]$ is transverse to $S^{3}$. Under the purity assumption $\left[Z_{1}\right] \cup\left[Z_{2}\right] \subset$ $\boldsymbol{C H}^{2}$. To show that $[Z]$ is transverse to $S^{3}$ it suffices to show that $\left[Z_{3}\right]$ is transverse to $S^{3}$. Recall that $Z_{3}$ is homeomorphic to $Z_{0} \times(-1,1)$, and $\left[Z_{0}\right]=[Z] \cap S^{3}$. It suffices to prove that $[T]$ is transverse to $S^{3}$ for any individual simplex $T$ in $Z_{3}$. But this follows immediately from Lemma 6.2.

\subsection{Pairing the Triangles}

In this section we analyze how the reflection pairings act on the triangles of $[Z]$. First we will see what happens to triangles in $\left[Z_{1}\right] \cup\left[Z_{2}\right]$ and then we will see what happens to triangles in $\left[Z_{3}\right]$.

Say that a border triangle is a triangle which is contained in two distinct pieces of $[Z]$. Say that an internal triangle is one which is contained in a single piece. For instance, the triangle with vertices $[\bullet(-3)],[\bullet 3],[\bullet 7]$ is contained only in $[A(0)]$. This triangle is paired to itself by $A_{0}$, but no other pairing reflection acts on it. All the internal triangles have this property.

Lemma 6.3 (Triangle Pairing Lemma) Modulo $K^{3}$, every border triangle of $[Z]$ is paired to two other border triangles.

Proof: Figure 6.5 shows the action of the reflection pairings on the vertices of $\partial\left[Z_{1}\right]$. The double arrow labelled by a $j$ shows the pairing action of the 
complex reflection which stabilizes $A_{j}$ and also the pairing action of the complex reflection which stabilizes $B_{j}$. (They both have the same action on these points.) Based on Figure 6.5 we deduce the action on the border triangles of $Z$ which are contained in $\left[Z_{1}\right] \cup\left[Z_{2}\right]$. Note that all the triangles on $\partial\left(\left[Z_{1}\right] \cup\left[Z_{2}\right]\right)$ are actually internal triangles, considered as triangles of $[Z]$. These triangles sit inside the even $[A]$ pieces, and are only exposed during our construction for the purposes of visualization.

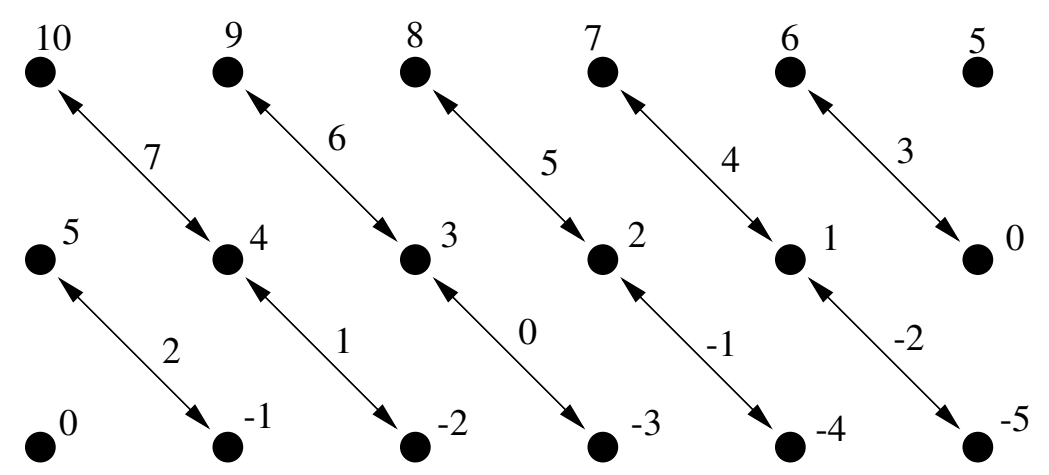

Figure 6.5

Since any border triangle belongs to two tetrahedra of $Z$, it is paired two other border triangles. Thus, we get a chain of pairings. Here we list 3 chains, using column vectors to represent triangles. Modulo $K^{3}$, which shifts the indices by +6 , the chains all have period 3 .

$$
\begin{aligned}
& {\left[\begin{array}{c}
\bullet 2 \\
\bullet 0 \\
\bullet(-2)
\end{array}\right] \stackrel{\overleftrightarrow{B_{1}}}{\longleftrightarrow}\left[\begin{array}{l}
\bullet 2 \\
\bullet 0 \\
\bullet 4
\end{array}\right] \quad \overleftrightarrow{B_{3}}\left[\begin{array}{l}
\bullet 2 \\
\bullet 6 \\
\bullet 4
\end{array}\right] \quad \overleftrightarrow{B_{5}}\left[\begin{array}{l}
\bullet 8 \\
\bullet 6 \\
\bullet 4
\end{array}\right] \ldots} \\
& {\left[\begin{array}{c}
\bullet(-2) \\
\bullet 2 \\
\bullet 3
\end{array}\right] \overleftrightarrow{B_{1}}\left[\begin{array}{c}
\bullet 4 \\
\bullet 2 \\
\bullet 3
\end{array}\right] \quad \overleftrightarrow{B_{5}}\left[\begin{array}{c}
\bullet 4 \\
\bullet 8 \\
\bullet 3
\end{array}\right] \quad \overleftrightarrow{A_{6}}\left[\begin{array}{c}
\bullet 4 \\
\bullet 8 \\
\bullet 9
\end{array}\right] \ldots} \\
& {\left[\begin{array}{c}
\bullet(-1) \\
\bullet(-2) \\
\bullet 3
\end{array}\right] \overleftrightarrow{B_{1}} \quad\left[\begin{array}{c}
\bullet(-1) \\
\bullet 4 \\
\bullet 3
\end{array}\right] \quad \overleftrightarrow{A_{6}} \quad\left[\begin{array}{c}
\bullet(-1) \\
\bullet 4 \\
\bullet 9
\end{array}\right] \quad \overleftrightarrow{A_{2}} \quad\left[\begin{array}{l}
\bullet 5 \\
\bullet 4 \\
\bullet 9
\end{array}\right] \ldots}
\end{aligned}
$$


All the other chains can be deduced from the action of $K$, which shifts the indices forward by +2 . In all, every border triangle in $\left[Z_{1}\right] \cup\left[Z_{2}\right]$ fits into 7 chains, each of which is equivalent via $K$ or $K^{2}$ to the ones listed. Here is an important conclusion for us: Modulo $K^{3}$ the border triangles in $\left[Z_{1}\right] \cup\left[Z_{2}\right]$ are identified in triples.

Every border triangle in $\left[Z_{3}\right]$ has vertices of both types. Thus, each border triangle in $\left[Z_{3}\right]$ corresponds to an edge of $\left[Z_{0}\right]$. These edges, of course, are plotted in Figure 4.9. The pairing reflections preserve the types of the vertices and thus respect the product structure of $\left[Z_{3}\right]$. Hence, the border triangles are paired together in precisely the same way that the edges of $\left[Z_{0}\right]$ are paired together. One can see from Figure 4.9 that the edges of $\left[Z_{0}\right]$ are paired together in triples, mod $K^{3}$. This follows from the relations in Lemma 4.5 , as we pointed out in $\S 5$. This completes the proof of the Pairing Lemma.

Corollary 6.4 The product of three consecutive pairings in any of our chains, considered as an element of $P U(2,1)$, is either $K^{3}$ or $K^{-3}$.

Proof: There are two ways to see this. First of all, we would do direct computations, referring to the chart in $\S 4.9$. For example: $B_{-1} B_{1} B_{3}=3.2 .212=$ $312=K^{-3}$. Alternatively, the action of such a product on a border triangle moves the border triangle by a power of $K^{3}$. It is easy to see that no border triangle is totally geodesic, and an element of $P U(2,1)$ is determined by its action on a non-totally-geodesic triangle.

The reader should compare Lemma 6.4 to Lemma 4.5.

\subsection{Pairing the Edges}

We define border edges and internal edges exactly we we did for triangles, with the change that a border edge belongs to 3 distinct pieces rather than 2. To justify this, we prove

Lemma 6.5 Every edge of $[Z]$ is contained in either 1 or 3 pieces.

Proof: For the edges in $\left[Z_{1}\right] \cup\left[Z_{2}\right]$ this statement can be gleaned from the chains listed in the previous section. For instance the first chain shows that 
$[\bullet 0 \bullet 4]$ belongs to $B_{1}$ and $B_{3}$. The second chain shows that $[\bullet 4 \bullet 8]$ belongs to $A_{6}$ and $B_{5}$. Hence $[\bullet 0 \bullet 4]$ belongs to $A_{2}$ in addition to $B_{1}$ and $B_{3}$. A routine inspection shows that this is it for $[\bullet 0 \bullet 4]$. The other edges have similar treatments. We omit the tedious details. Recall that we have deleted the pure simplices from $\left[Z_{3}\right]$. Similar to the situation for triangles, the edges of $\left[Z_{3}\right]$ are in bijection with the vertices of $\left[Z_{0}\right]$. Looking at Figure 4.9 we can see that each vertex belongs to three tiles. Hence, each edge of $\left[Z_{3}\right]$ belongs to 3 pieces. This covers all the cases.

Lemma 6.6 (Edge Pairing Lemma) Modulo $K^{3}$, every border edge of $[Z]$ is paired to one other order edge.

Proof: The proof is essentially the same as for the case of triangles. For the case of border edges in $\left[Z_{1}\right] \cup\left[Z_{2}\right]$ we can get the information from our chains. For instance, looking at the top line of the first chain, we see that $[\bullet 2 \bullet 0]$ is paired to itself and to $[\bullet 2 \bullet 6]$. This edge is paired to itself and to $[\bullet 8 \bullet 6]$, but this last edge is equivalent to $[\bullet 2 \bullet 0]$. To figure out the pairings of edges in $\left[Z_{3}\right]$ we just have to look at the pairings of the vertices in Figure 4.9 .

We say that two edges $e_{1}, e_{2} \in Z$ are equivalent if there is some $\gamma \in$ $S U(2,1)$ such that $\gamma\left(e_{1}\right)$ is a multiple of $e_{2}$.

Lemma 6.7 Every edge of $Z_{3}$ is equivalent to one of the three edges

$$
\left[\begin{array}{c}
\bullet 1 \\
\circ(-7)
\end{array}\right] ; \quad\left[\begin{array}{l}
\bullet 1 \\
\circ 4
\end{array}\right] ; \quad\left[\begin{array}{l}
\bullet 1 \\
\circ 9
\end{array}\right]
$$

These three edges are pairwise inequivalent.

Proof: Each edge of $Z_{3}$ is contained in three pieces, and one of them has the form $A(2 m+1)$. (Compare the vertices in Figure 4.9.) Hence, each edge of $Z_{3}$ is equivalent to an edge which is contained in $A(1)$. In Figure 6.0 we see that there are 4 edges of $Z_{3}$ contained in $A(1)$. (Recall that the pure edges have been deleted.) Three of the edges are the ones listed above, and the fourth one is paired to $[\bullet 1 \circ 4]$ by the pairing reflection $A_{1}$. As for the inequilvalence, we compute explicitly that the values $\delta(\bullet 1, \circ j)$ are pairwise unequal for $j=-7,4,9$. Here $\delta$ is as in Equation 6 . 


\section{The Proof Modulo the Assumptions}

\subsection{Overview}

We continue to work with the complex $Z$. As in $\S 6$ we assume that $Z$ satisfies the 3 assumptions listed in $\S 6.1$.

The Combinatorial Lemma of $\S 6$ says that $\left([Z],\left[Z_{0}\right]\right)$ is homeomorphic to $\left(D, S^{1}\right) \times \boldsymbol{R}$. Here $D$ is the open disk of radius 2. Recall that $K([Z])=[Z]$. Being loxodromic, $K$ acts properly on $S^{3}-\left[E_{0} K\right]-\left[E_{\infty} K\right]$, so that $\left[E_{0} K\right]$ and $\left[E_{\infty} K\right]$ are the two accumulation points of $\left[Z_{0}\right]$ which are not contained in $\left[Z_{0}\right]$. It follows from the piecewise analytic nature of the projectivization map that $\left[Z_{0}\right] \cup\left[E_{0} K\right] \cup\left[E_{\infty} K\right]$ is a tamely embedded 2-sphere whose complement consists of two components, both 3-balls.

Using the fact that $[Z]$ is homeomorphic to $D \times \boldsymbol{R}$, together with the fact that $[Z]$ has a product structure in the neighborhood of $\left[Z_{0}\right]$, we see that $\left[Z_{-}\right]$ is an open, tamely embedded 3-ball. Moreover, $\boldsymbol{C} \boldsymbol{H}^{3}-\left[Z_{-}\right]$consists of two open components, both 4-balls. Each component of $\boldsymbol{C H}^{2}-\left[Z_{-}\right]$extends to one of the components of $S^{3}-\left[Z_{0}\right]$, in an obvious way. In the next section we will choose the "correct" component $\Delta_{-}$of $\boldsymbol{C H}^{2}-\left[Z_{-}\right]$. Let $\Delta_{0}$ be the corresponding component of $S^{3}-\left(\left[Z_{0}\right] \cup\left[E_{0} K\right] \cup\left[E_{\infty} K\right]\right)$.

We define

$$
Q_{-}=\left(\Delta_{-} \cup\left[Z_{-}\right]\right) / K^{3} .
$$

We let $\sim$ be the equivalence relation on $Q_{-}$induced by the reflection pairings. We define

$$
O=Q_{-} / \sim .
$$

It is easy to see that $O$ is a Hausdorff space. Since $\sim$ introduces no relations on $\Delta_{-} / K^{3}$, the space $O$ contains an open dense subset which is locally isometric to $\boldsymbol{C} \boldsymbol{H}^{2}$. We put a metric on all of $O$ by taking the completion of the complex hyperbolic metric defined on this subset. We have the quotient map $Z \rightarrow O$. Let $O^{0}$ be the image of the 0 -skeleton of $Z$ in $O$. It is not hard to see that $O^{0}$ consists of the 6 points, the images of $\bullet 1, \ldots, \bullet 6$.

Lemma 7.1 (Orbifold) Given the assumptions, the following is true: Every point of $O-O^{0}$ has a neighborhood which is locally isometric either to $\boldsymbol{C H}^{2}$ or to $\boldsymbol{C H}^{2} / I$, wnere I is a complex reflection.

The Orbifold Lemma implies that $O$ is a complex hyperbolic orbifold. Each of the finitely many unanalyzed points is the apex of a cone on a 
compact spherical orbifold; and the orbifold structure extends right across the apex. A vastly more general result is established in [T2, Codimension 2 Conditions Suffice].

The following corollary fits into the context of fundamental domains for coset decompositions. Compare $[\mathbf{B}, \S 9.6]$.

Corollary 7.2 Given the assumptions, the following is true:

1. $O=\boldsymbol{C H}^{2} / \Gamma(4,7)$.

2. $\boldsymbol{C} \boldsymbol{H}^{2}$ is tiled by $\Gamma(4,7)$-translates of $\Omega$.

3. The stabilizer subgroup of $\Omega$, in $\Gamma(4,7)$, is generated by $K^{3}$.

Proof: Every complex hyperbolic orbifold is the quotient of $\boldsymbol{C H}^{2}$ by a discrete group. (This is generally true for orbifolds modelled on analytic spaces. See $[\mathbf{T 2}]$.) Thus, $O=\boldsymbol{C H}^{2} / \Gamma^{\prime}$. It is easy to see that $\Gamma^{\prime}$ is generated by the reflection pairings, and these in turn generate $\Gamma(4,7)$. Hence $\Gamma^{\prime}=$ $\Gamma(4,7)$.

Let $\pi: \boldsymbol{C H}^{2} \rightarrow O$ be the universal covering map. Since $\sim$ makes no identifications on $\Delta_{-}$, we have an embedding $\Delta_{-} / K^{3} \hookrightarrow O$. Since $\Delta_{-}$is simply connected we can identify one component of $\pi^{-1}\left(\Delta_{-} / K^{3}\right)$ with $\Delta_{-}$ itself. Moreover, every element of $\Gamma(4,7)$ either stabilizes $\Delta_{-}$or moves it disjointly from itself. By construction, the translates of $\Delta_{-}$are dense in $\boldsymbol{C H}^{2}$. Hence, the translates of $\Delta_{-} \cup\left[Z_{-}\right]$tile $\boldsymbol{C} \boldsymbol{H}^{2}$.

Since $K^{3}$ is the product of reflection pairings-see Lemma 4.5 -we know that $K^{3}$ is contained in the stabilizer subgroup of $\Delta_{-}$. By construction, the stabilizer subgroup of $\Delta_{-}$in $P U(2,1)$ is the cyclic subgroup generated by $K$. If $K \subset \Gamma(4,7)$ then $\Delta_{-} / K^{3}$ would not embed into $O$. In summary, the stabilizer subgroup of $\Delta_{-}$is exactly the cyclic subgroup generated by $K^{3}$.

Under our assumptions, the tiling of $\boldsymbol{C H}^{2}$ by translates of $\Delta_{-} \cup\left[Z_{-}\right]$ extends to a tiling of $\Omega(4,7)$ by translates of $\Delta_{0} \cup\left[Z_{0}\right]$. This follows from the fact that $[Z]$ has a product structure in a neighborhood of $\left[Z_{0}\right]$.

We have stated our results in a conditional way because $Z$ does not satisfy the Homeomorphism Assumption, as we will see in $\S 8$. However, the replacement $Z^{\prime}$ satisfies all the assumptions and the proofs for $Z^{\prime}$ work the same way they (would) work for $Z$. 


\subsection{Proof of the Orbifold Lemma}

We first explain how to pick the "correct" component of $\boldsymbol{C H}^{2}-\left[Z_{-}\right]$. Our definition requires us to choose a border triangle $\sigma$ but we will see that our definition is independent of this choice.

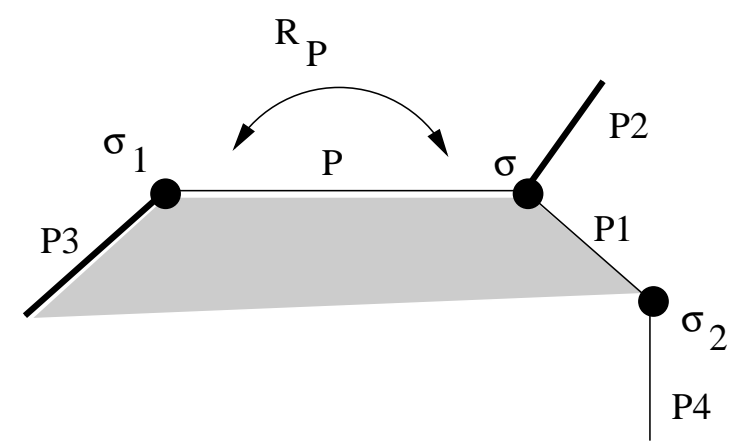

Figure 7.1

Let $P$ and $P_{1}$ be the two pieces containing $\sigma$. Let $R_{P}$ be the reflection pairing associated to $P$. Let $\sigma_{1}=R_{P}(\sigma) \subset P$. Let $P_{3}$ be the other piece which contains $\sigma_{1}$. Let $P_{2}=R_{P}\left(P_{3}\right)$. See Figure 7.1. We define $\Delta_{-}$to be the component of $\boldsymbol{C H}^{2}-\left[Z_{-}\right]$which is separated from $P_{2}$ by $[Z]$. This is the shaded region in Figure 7.1.

Lemma 7.3 Switching the roles of $P$ and $P_{1}$ does not change the definition of $\Delta_{-}$.

Proof: If we interchanged the roles of $P$ and $P_{1}$ then we would get $P_{2}=$ $R_{P_{1}}\left(P_{4}\right)$, where $P_{4} \cap P_{1}=\sigma_{2}$, as shown in Figure 7.1. It suffices to show that $P_{2}=R_{P} R_{P_{1}}\left(P_{4}\right)$. We compute

$$
R_{P} R_{P_{1}}\left(P_{4}\right)=R_{P} R_{P_{1}} R_{P_{4}}\left(P_{4}\right)=K^{-3}\left(P_{4}\right)=P_{1} .
$$

This follows from Lemma 6.4 or Lemma 4.5, depending in the choice of $\sigma$.

Lemma 7.4 The definition of $\Delta_{-}$does not depend on the choice of $\sigma$.

Proof: For the sake of argument let's write $\Delta_{-}(\sigma)$, etc. to denote the dependence on $\sigma$. Every two border tiles $\sigma$ and $\tau$ can be joined in a finite 
sequence $\sigma=\sigma_{0}, \ldots, \sigma_{n}=\tau$ such that $\sigma_{j}$ and $\sigma_{j+1}$ share an edge. Thus, it suffices to consider the case when two border triangles share an edge.

If $\sigma$ and $\tau$ share an edge, then so do $\sigma_{1}$ and $\tau_{1}$. Hence, so do $P_{3}(\sigma)$ and $P_{3}(\tau)$. Hence, so do $P_{2}(\sigma)$ and $P_{2}(\tau)$. But then both of these sets lie in the same component of $\boldsymbol{C H}^{2}-[Z]$.

Note that the map $R_{P}$ locally interchanges the two sides of $\left[Z^{\prime}\right]$. For this reason, $\Delta_{-}$has the following property. For each piece $[P]$ there is an open neighborhood $U_{P}$ such that $\left[P_{-}\right] \subset U_{P}$ and

$$
R_{P}\left(U_{P} \cap \Delta_{-}\right) \cap \Delta_{-}=\emptyset .
$$

This is one of the hypotheses of the Poincarè' theorem on Fundamental Polyhedra. See $[\mathbf{B}]$.

Now that we have chosen $\Delta_{-}$we define

$$
O_{2}=\Delta_{-} \cup\left[Z_{-}\right] ; \quad O_{1}=O_{2} / K^{3} .
$$

We have quotient maps

$$
\mathrm{O}_{2} \quad \overrightarrow{\pi_{2}} \quad \mathrm{O}_{1} \quad \overrightarrow{\pi_{1}} \quad O
$$

We want to analyze the neighborhood of a point $x \in O$. We choose lifts $x_{j} \in O_{j}$ so that $\pi_{2}\left(x_{2}\right)=x_{1}$ and $\pi_{1}\left(x_{1}\right)=x$.

$\pi_{2}$ is a covering map and $\pi_{1}$ is an injection on $\pi_{2}\left(\Delta_{-}\right)$. Hence, $x$ has a neighborhood locally isometric to $\boldsymbol{C H}^{2}$ if $x_{2} \in \Delta_{-}$. If $x_{2}$ is contained in the interior of a piece of $\left[Z_{1}\right]$ then only one reflection pairing acts on $x_{2}$. Therefore, the neighborhood of $x$ in $O$ is locally isometric either to $\boldsymbol{C H}^{2}$ or to $\boldsymbol{C H}^{2} / I$. Here $I$ is a complex reflection. Henceforth we assume $x_{2}$ is contained in more than one piece of $[Z]$. There are two cases, the first of which is crucial.

Case 1: Suppose that $x_{2}$ is contained in the interior of a border triangle $\sigma$. Let $\beta$ be a small metric ball in $\boldsymbol{C H}^{2}$ centered at $x_{2}$. We choose $\beta$ so small that it is disjoint from all its $K$-translates. The argument in the proof of Lemma 7.3 shows that $P$ and $P_{1}$ are both pieces of $R_{P_{1}}\left(\left[\Delta_{-}\right]\right)$. Hence, the sets

$$
S_{1}=\left[\Delta_{-}\right] \cap \beta ; \quad S_{2}=R_{P}\left(\left[\Delta_{-}\right]\right) \cap \beta ; \quad S_{3}=R_{P_{1}}\left(\left[\Delta_{-}\right]\right) \cap \beta .
$$

partition $\beta$ exactly, as shown in Figure 7.2. 


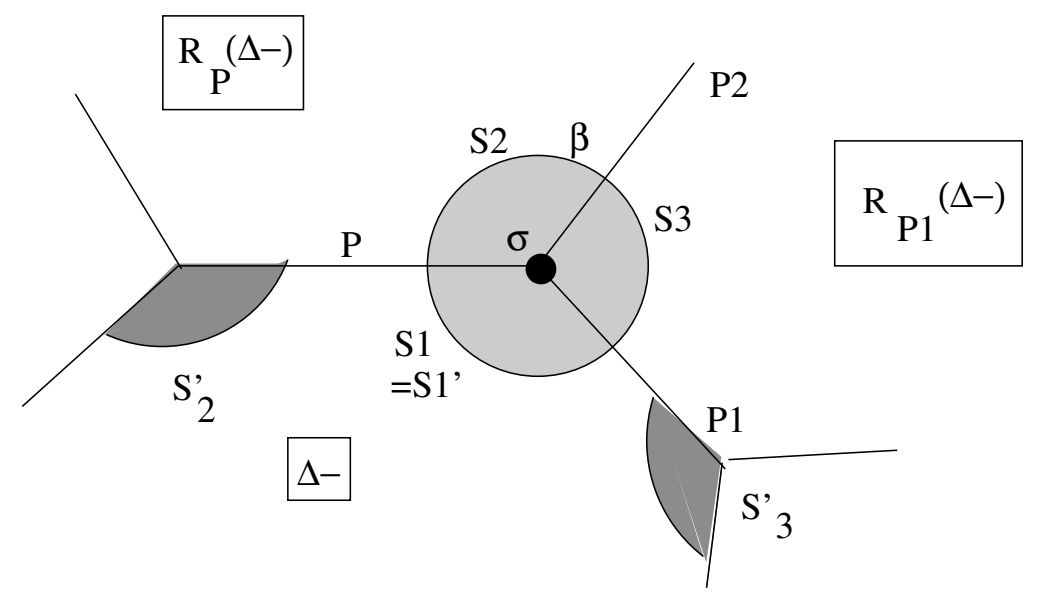

Figure 7.2

From the Triangle Pairing Lemma there are three triangles in $O_{1}$, one of them $\pi_{1}(\sigma)$, which are paired together by the equivalence relation $\sim$. The three sets

$$
S_{1}^{\prime}=\pi_{2}\left(S_{1}\right) ; \quad S_{2}^{\prime}=\pi_{2}\left(R_{P}^{-1}\left(S_{2}\right)\right) ; \quad S_{3}^{\prime}=\pi_{2}\left(R_{P_{1}}^{-1}\left(S_{2}\right)\right)
$$

are neighborhoods of our three triangles in $O_{1}$. Moreover, $\pi_{1}$ maps $S_{1}^{\prime} \cup S_{2}^{\prime} \cup S_{3}^{\prime}$ onto an isometric copy of $\beta$, and this isometric copy is a neighborhood of $x$ in $O$.

Case 2: In this case $x_{2}$ is contained in the interior of a border edge of $\left[Z_{-}\right]$. We remark that this challenging case is probably unnecessary. With small modifications, our situation probably would fit into the machinery of [T2, Codimension Two Conditions Suffice], and Case 1 is enough.

Rather than work out the general case in the abstract we will work out the particular example where the border edge is $[\bullet 2, \bullet 7]$. Every other example has the same analysis, on account of the uniformity of the results in the Edge Pairing Lemma, the Triangle Pairing Lemma, Lemma 4.5 and Lemma 6.4.

Looking at Figure 7.0, we see that

$$
[\bullet 2, \bullet 7]=[A(0)] \cap[A(4)] \cap[B(5)] .
$$

Modulo $K^{3}$ the only edge paired to $[\bullet 2, \bullet 7]$ is $[\bullet 1, \bullet 2]$. The pairing is effected by $A_{4}$. Looking at Figure 7.0 we see that

$$
[\bullet 1, \bullet 2]=[A(4)] \cap[B(3)] \cap[B(-1)] .
$$


The pairing $A_{0}$ fixes $[\bullet 2, \bullet 7]$ and the pairing $B_{3}$ fixes $[\bullet 1, \bullet 2]$. We have $A_{0} A_{4} B_{-1}=K^{-3}=A_{4} B_{3} B_{-1}$, from Equations 78, 79 and Lemma 6.4. Hence $A_{0} A_{4}=A_{4} B_{3}$.

Let $\beta$ be a small metric ball about $x_{2}$, as in the previous case. $[\bullet 2, \bullet 7] \cap \beta$ is contained in the boundary of the pieces:

$$
\begin{array}{r}
S_{1}=\beta \cap\left[\Delta_{-}\right] ; \quad S_{2}=\beta \cap A_{0}\left(\left[\Delta_{-}\right]\right) ; \\
S_{3}=\beta \cap A_{4}\left(\left[\Delta_{-}\right]\right) ; \quad S_{4}=\beta \cap A_{4} B_{3}\left(\left[\Delta_{-}\right]\right) .
\end{array}
$$

Let's compute the intersection $S_{i j}=S_{i} \cap S_{j}$ :

$$
\begin{array}{r}
S_{12}=\beta \cap[A(0)] ; \quad S_{13}=\beta \cap[A(4)] ; \quad S_{14}=\beta \cap[B(5)] ; \\
S_{23}=\beta \cap A_{4}([B(5)]) \quad S_{24}=\beta \cap A_{0}([A(4)]) \quad S_{34}=\beta \cap A_{4}([B(3)]) .
\end{array}
$$

The third equation is the most complicated. It follows from

$$
A_{4} B_{3}([B(-1)])=A_{4} B_{3} B_{-1}([B(-1)])=K^{3}([B(-1)])=[B(5)],
$$

which in turn follows from Lemma 6.4. To summarize, our calculations show that $S_{i} \cap S_{j}=\beta \cap[P]$, where $[P]$ is either a piece of $[Z]$ or the translate of a piece. This pattern of intersection implies that the pieces $S_{1}, S_{2}, S_{3}, S_{4}$ fit together to exactly partition $\beta$.

There is a nice 3-dimensional model of the partition of $\beta$. Let $\tau$ be a tetrahedron. Let $s_{1}, s_{2}, s_{3}, s_{4}$ be the cones to the barycenter of the faces of $\tau$. Thus $\tau=s_{1} \cup s_{2} \cup s_{3} \cup s_{4}$. The pattern of intersection of the $s_{j}$ is the same as the pattern of intersection of the $S_{j}$. The complex reflection $A_{0}$ interchanges $S_{1}$ and $S_{2}$, and also interchanges $S_{3}$ and $S_{4}$. In our 3dimensional combinatorial model, this reflection corresponds to a 180 degree rotation about the edge joining the midpoint of $\partial s_{1} \cap \partial s_{2}$ to the midpoint of $\partial s_{3} \cap \partial s_{4}$. To get the actual 4-dimensional picture, we take the product of the three dimensional model with an interval.

Define

$$
S_{12}^{\prime}=S_{1}=A_{0}\left(S_{2}\right) ; \quad S_{34}^{\prime}=A_{4}\left(S_{3}\right)=B_{3} A_{4}\left(S_{4}\right) .
$$

The images $\pi_{2}\left(S_{12}^{\prime}\right)$ and $\pi_{2}\left(S_{12}^{\prime}\right)$ respectively are neighborhoods of $\pi_{2}([\bullet 2, \bullet 7])$ and $\pi_{2}([\bullet 1, \bullet 2])$. Hence $N=\pi_{1}\left(S_{12}^{\prime} \cup S_{34}^{\prime}\right)$ is a neighborhood of $x$ in $O$. From everything we have said, there is a degree 2 branched covering $\beta \rightarrow N$ which commutes with $A_{0}$, maps $S_{1}$ and $S_{2}$ to $\pi_{1}\left(S_{12}^{\prime}\right)$ and maps $S_{3}$ and $S_{4}$ to $\pi_{1}\left(S_{34}^{\prime}\right)$. This shows that $N$ is isometric to $\beta / A_{0}$. 


\section{Refining the Complex}

\subsection{The Flaw}

The Homeomorphism Assumption, used in $\S 6$ and $\S 7$, is flawed. It turns out that $[T]$ is not embedded for some of the tetrahedra $T$ in $Z$. Figure 8.1 illustrates the flaw. Let $\alpha_{1}$ be the complex line fixed by the reflection pairing $A_{1}$, acting projectively. Looking at Figure 7.0, we see that $\alpha_{1}$ contains the points $[\bullet 1],[\circ-7]$ and $[\circ 9]$, and bisects $[A(1)]$ in a pair of triangles. Figure 8.1 shows a computer plot of $\alpha_{1} \cap[A(1)]$. The grey circle is $\alpha_{1} \cap S^{3}$. If $[A(1)]$ was embedded then $[A(1) \cap L]$ would be a pair of embedded triangles.

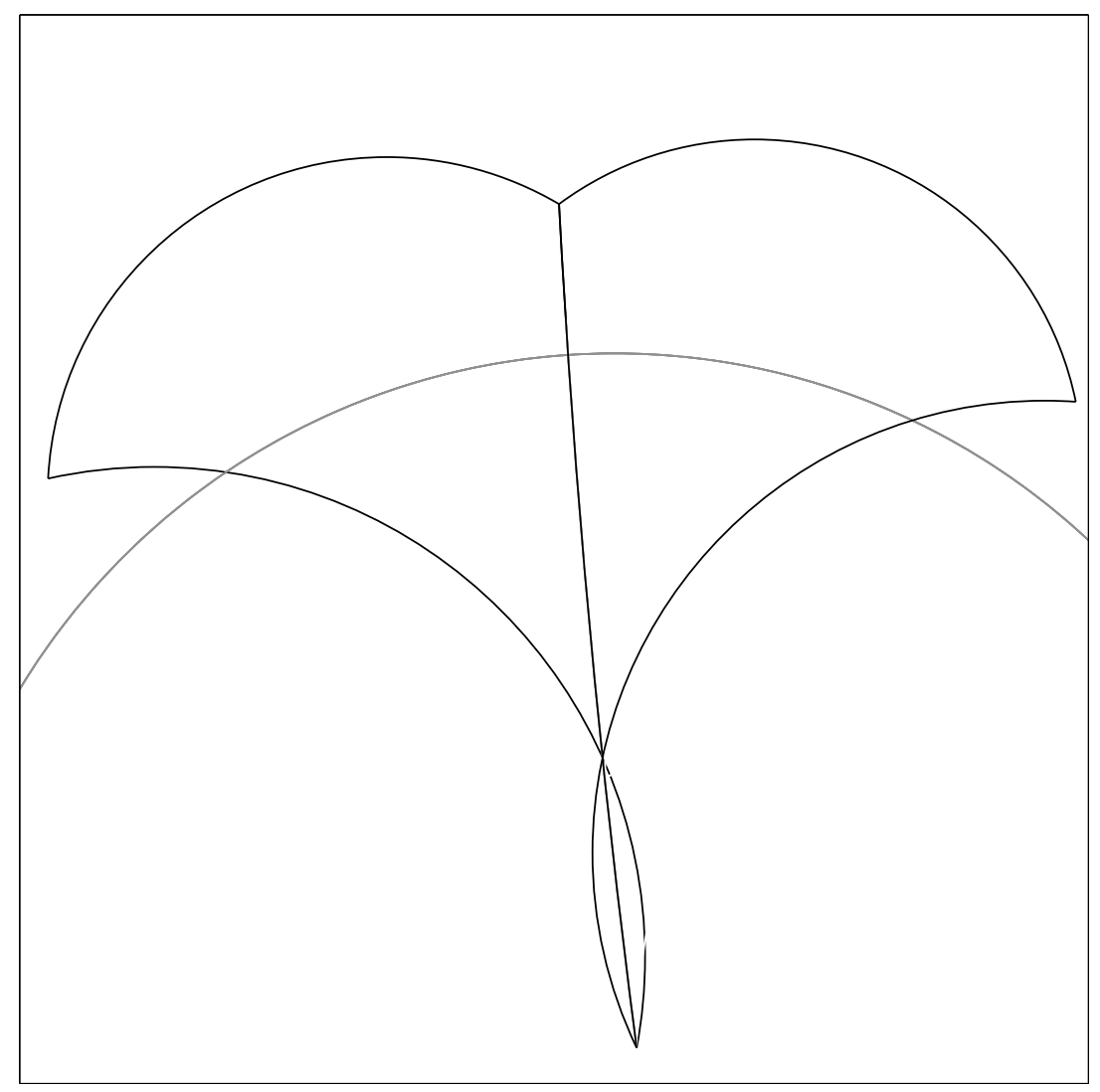

Figure 8.1

In this chapter we will replace $Z$ by $Z^{\prime}$. In $\S 9$ we will prove that $Z^{\prime}$ satisfies the three assumptions of $\S 6.1$. Figure 8.3 shows the slice of $Z^{\prime}$ which corresponds to Figure 8.1. 


\subsection{The Basic Constructions}

We begin with two definitions which just use the real structure of $\boldsymbol{C}^{2,1}$. If $S \subset C^{2,1}$ is a finite polyhedron we let $S^{0}$ be the set of vertices of $S$. We let $\underline{S}$ be the average of the vectors in $S^{0}$. We call $\underline{S}$ the barycenter of $S$. A map $\phi: \boldsymbol{C}^{2,1} \rightarrow \boldsymbol{C}^{2,1}$ is real affine if $\phi$ commutes with the operation of taking barycenters.

Here is a canonical way to partition a 3-dimensional convex polyhedron $P \subset C^{2,1}$ into tetrahedra: Let $\sigma$ be a face of $P$. If $\sigma$ is a triangle, we do nothing. If $\sigma$ is not a triangle, we cone the edges of $\sigma$ to the barycenter of $\sigma$, thereby subdividing $\sigma$ into triangular pieces. We subdivide all faces of $\partial P$ in this manner and then cone the union of all these triangles to the barycenter of $P$. In one exceptional case, when $P$ is a tetrahedron, we redefine $P^{\#}=P$. In general, $P^{\#}$ has as much symmetry as $P$ does. $\left(P^{\#}\right.$ is closely related to the barycentric subdivision of $P$, but has fewer tetrahedra. For details on barycentric subdivision, see [Sp, p. 123].)

Suppose that $\phi: P^{0} \rightarrow C^{2,1}$ is any map. There is a unique extension $\phi^{\#}: P^{\#} \rightarrow C^{2,1}$ such that

1. $\phi^{\#}(\underline{P})=\underline{\phi\left(P^{0}\right)}$.

2. If $\sigma$ is a face of $P$ then then $\phi^{\#}(\underline{\sigma})=\underline{\phi\left(\sigma^{0}\right)}$.

3. The restriction of $\phi^{\#}$ to any simplex of $P^{\#}$ is real affine.

The conditions above give the recipe for the construction of $\phi^{\#}$ from $\phi$.

Suppose that $T \subset C^{2,1}$ is a tetrahedron which has both positive and negative vertices, but no null vertices. Let $T_{-}$and $T_{+}$be the set of negative and positive vertices respectively. Figure 8.2 shows two cases. The vertices of $T_{-}$are colored black and the vertices of $T_{+}$are colored white. Say that a mixed edge of $T$ is an edge which connects a black vertex to a white vertex. Let $e_{1}, \ldots, e_{k}$ be the mixed edges. Here $k$ is either 3 or 4 . Let $\Pi_{0}, \Pi_{1}, \Pi_{2}$ be three parallel planes which separate $T_{-}$from $T_{+}$, ordered so that $\Pi_{0}$ is closest to $T_{-}$and $\Pi_{2}$ is closest to $T_{+}$. The particular choice of these planes is irrelevant for our combinatorial purposes.

For $j=0,1,2$ we define

$$
T_{j}=\bigcup_{i=1}^{k}\left(e_{i} \cap \Pi_{j}\right) .
$$



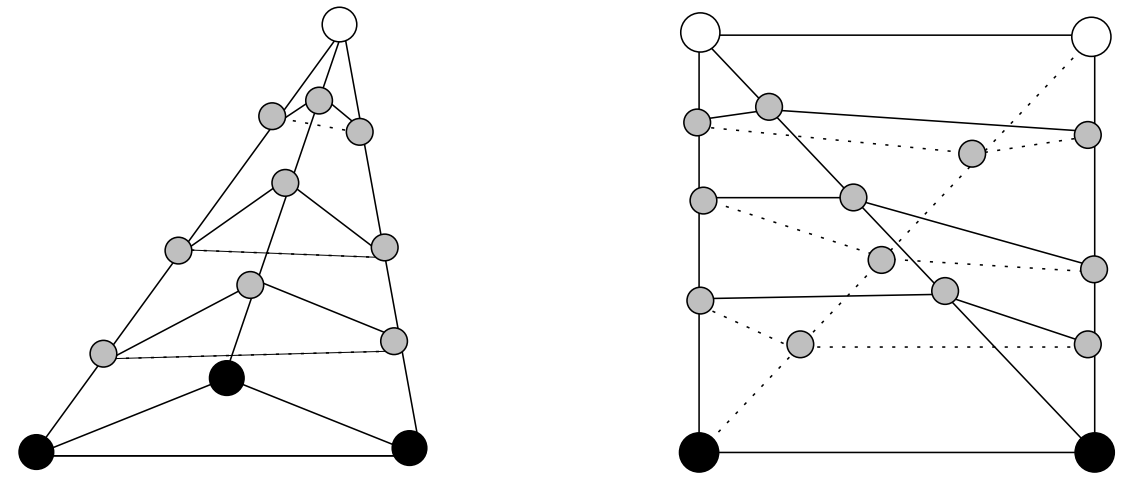

Figure 8.2

The point of $T_{j}$ are coplanar. The grey dots in Figure 8.2 are the points of $T_{0} \cup T_{1} \cup T_{2}$. For $j=-1,0,1$ we let $T^{j}$ be the convex hull of $T_{j-1} \cup T_{j}$. Here $T_{-1}$ is interpreted as $T_{-}$. Combinatorially, $T^{j}$ is either a cube, a tetrahedron, or the product of a triangle with an interval.

To each mixed edge $e_{i}$ we assign three complex numbers $\mu_{j}\left(e_{i}\right), j=1,2,3$. We define

$$
\phi\left(e_{i} \cap \Pi_{j}\right)=\left(1-\left|\mu_{j}\left(e_{i}\right)\right|\right) e_{i,-}+\mu_{j} e_{i,+} .
$$

Here $e_{i,-}$ is the negative endpoint of $e_{i}$ and $e_{i,+}$ is the positive endpoint. We also define $\phi$ to be the identity on $T_{-}$. We let $\phi_{j}$ be the restriction of $\phi$ to the 0 -skeleton of $T^{j}$, namely $T_{j-1} \cup T_{j}$. Finally, we define

$$
T_{j}^{\prime}=\phi_{j}^{\#}\left(T^{j}\right) ; \quad T^{\prime}=\bigcup_{j=0}^{2} T_{j}^{\prime} .
$$

The definition of $T^{\prime}$ depends on $\mu$. By construction $T^{\prime}$ is a finite union of tetrahedra. Note, however, that $T^{\prime}$ need not be a subset of $T$. Indeed, $T^{\prime}$ may not even lie in a 3-real-dimensional subspace of $\boldsymbol{C}^{2,1}$.

We will be interested in the case when

1. $\mu_{2}\left(e_{i}\right)$ and $\mu_{3}\left(e_{i}\right)$ are real for all $i$.

2. $0<\mu_{2}\left(e_{i}\right), \mu_{3}\left(e_{i}\right)<1$ for all $i$.

In these cases, $\phi^{3}$ maps $T^{2} \cup T^{3}$ into $T$ and $T_{2}^{\prime} \subset T$. The nontrivial part of the perturbation in this case takes place in $T_{0}^{\prime}$ and $T_{1}^{\prime}$. 


\subsection{Perturbing the Complex}

Recall from $\S 6$ that $Z=Z_{1} \cup Z_{2} \cup Z_{3}$. Our refinement of $Z$ takes place entirely within $Z_{3}$. Recall that every tetrahedron of $Z_{3}$ satisfies the hypotheses in the previous section. We have deleted all the pure edges from $Z_{3}$ and so every edge of $Z_{3}$ connects a negative vector to a positive vector.

To each edge $e$ of $Z_{3}$ we assign a vector $\mu(e) \in \boldsymbol{C} \times \boldsymbol{R}^{2}$. Once we make this assignment, we replace each tetrahedron $T$ of $Z_{3}$ by $T^{\prime}$. Here $T^{\prime}$ is defined using the restriction of $\mu$ to the mixed edges of $T$. We obtain $Z_{3}^{\prime}$ by replacing each tetrahedron $T$ of $Z_{3}$ by $T^{\prime}$. As with $Z_{3}$, we delete the pure positive simplices from $Z_{3}^{\prime}$. Finally, we let $Z^{\prime}=Z_{1} \cup Z_{2} \cup Z_{3}^{\prime}$.

For notational convenience we set $T^{\prime}=T$ if $T$ is a tetrahedron of $Z_{1}$ or $Z_{2}$. Thus, $Z^{\prime}$ is obtained from $Z$ by replacing each tetrahedron $T$ by $T^{\prime}$. If $P$ is a piece of $Z$ we let $P^{\prime}$ be the corresponding union of tetrahedra in $Z^{\prime}$. We call $P^{\prime}$ a piece of $Z^{\prime}$. Thus, the pieces of $Z^{\prime}$ are $A^{\prime}(n)$ and $B^{\prime}(n)$, where $n$ ranges in $\boldsymbol{Z}$.

We would like to define $\mu$ so that $Z^{\prime}$ has all the same symmetries that $Z$ does. First of all, we must have $\mu\left(e_{1}\right)=\mu\left(e_{2}\right)$ if $e_{1}$ and $e_{2}$ are equivalent in the sense Lemma 6.7. This it suffices to specify $\mu$ on the three vectors listed in Lemma 6.7. Up to a sign, the antiholomorphic map $I_{1} J B_{1}$ interchanges the first and third vectors of Lemma 6.7 and preserves the second one. Thus, we must take

$$
\mu\left[\begin{array}{c}
\bullet 1 \\
\circ(-7)
\end{array}\right]=\bar{\mu}\left[\begin{array}{c}
\bullet 1 \\
\circ 9
\end{array}\right] ; \quad \mu\left[\begin{array}{l}
\bullet 1 \\
\circ 4
\end{array}\right] \in \boldsymbol{R}^{3}
$$

With $\delta$ as in Equation 6 , the quantity $\delta(\bullet 1, \circ 9)$ is not real, so there is no antiholomorphic map which preserves $[\bullet \mathbf{1}, \circ 9]$ up to a sign. Thus, there is no incompatibility in taking $\mu_{1}([\bullet 1, \circ 9]) \in \boldsymbol{C}-\boldsymbol{R}$.

Here is the upshot: To define $Z^{\prime}$ in such a way that it retains all the symmetries of $Z$ we must

1. specify $\mu([\bullet 1, \circ 2]) \in \boldsymbol{R}^{3}$ and $\mu([\bullet 1, \circ 9]) \in \boldsymbol{C} \times \boldsymbol{R}^{2}$;

2. determine $\mu([\bullet 1, \circ(-7)]$ by Equation 95 .

3. set $\mu([\bullet 1, \circ(-2)])=\mu([\bullet 1, \circ 4])$, because the reflection pairing $A_{1}$ interchanges these two edges, up to sign.

4. extend $\mu$ to all the other edges of $Z_{4}$ using the action of $K$. 
Compare the proof of Lemma 6.7.

We have 7 free parameters. A lot of trial and error led to the choices

$$
\mu\left[\begin{array}{l}
\bullet 1 \\
\circ 4
\end{array}\right]=(.30, .55, .85) \quad \mu\left[\begin{array}{l}
\bullet 1 \\
\circ 9
\end{array}\right]=(-.23-.11 \sqrt{-1}, .45, .92)
$$

\subsection{The End of the Proof}

We compute directly, for any tetrahedron $T \in A(0) \cup A(1) \cup B(0) \cup B(1)$,

1. All the vertices of $T_{1}^{\prime}$ and $T_{2}^{\prime}$ are negative.

2. All the vertices of $T_{3}^{\prime}-T_{2}^{\prime}$ are positive.

There is not much to say about this computation. We just list out the vectors and compute their norms, using interval arithmetic to control the round-off error, as explained in $\S 10.1$. By symmetry every tetrahedron $T$ of $Z$ has the two properties above. Compare Figures 8.3-8.5 below. We have already remarked at the end of $\S 8.2$ that $T_{3}^{\prime} \subset T$. Combining this property with the Purity Assumption, we see that $T$ and $T^{\prime}$ agree in a neighborhood of $N_{0}$. Hence $Z$ and $Z^{\prime}$ agree in a neighborhood of $N_{0}$.

From a combinatorial point of view, $Z^{\prime}$ is just a refinement of $Z$. The pieces fit together in exactly the same way. Lemmas 4.1-4.4 remain true for

$Z^{\prime}$ due to the symmetric way we picked $\mu$. Because $Z$ and $Z^{\prime}$ enjoy exacrly the same symmetries, all the results go of $\S 7$ go through for $Z^{\prime}$ in place of $Z$. In $\S 9$ we will prove that $Z^{\prime}$ satisfies the assumptions listed in $\S 7.1$. Combining this fact with the results in $\S 7$ completes the proof of Theorem 1.1.

\subsection{Some Pictures}

Figure 8.3 shows a computer plot of $\left[A^{\prime}(1)\right] \cap \alpha_{1}$. All the triangles appear to be (and are) embedded, indicating that all the tetrahedra in sight are embedded. A comparison of Figures 8.1 and 8.3 shows that these figures agree in a neighborhood of the grey circle, as desired. 


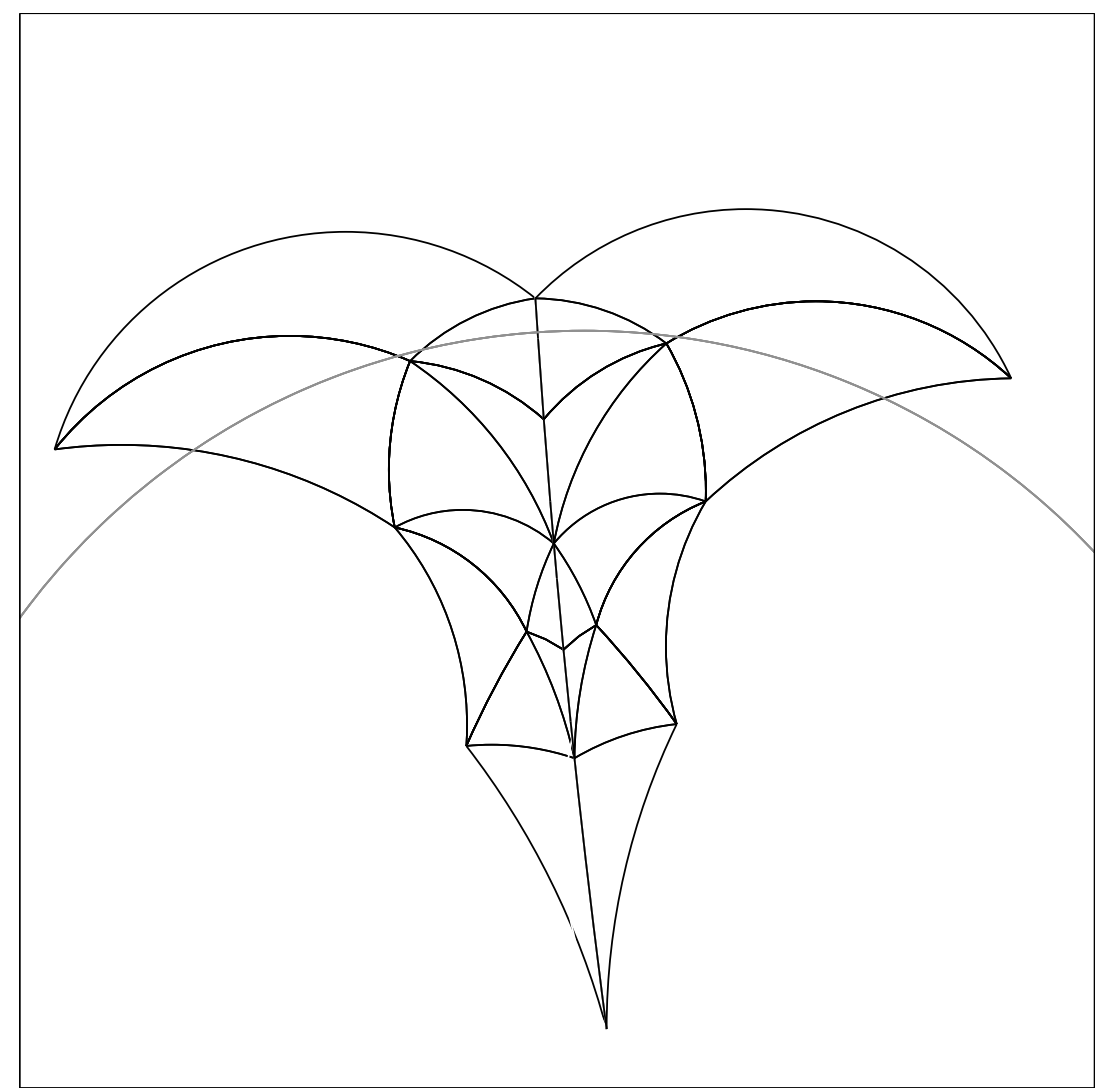

Figure 8.3

Figure 8.4 plots $\left.\left[A^{\prime}(0)\right] \cap \alpha_{0}\right]$. Here $\alpha_{0}$ is the complex line fixed by the reflection pairing $A_{0}$. Again, all triangles appear to be (and are) embedded. Figure 8.5 plots $\left[B^{\prime}(0)\right] \cap \beta_{0}$, where $\beta_{0}$ is the complex line fixed by $B_{0}$. 


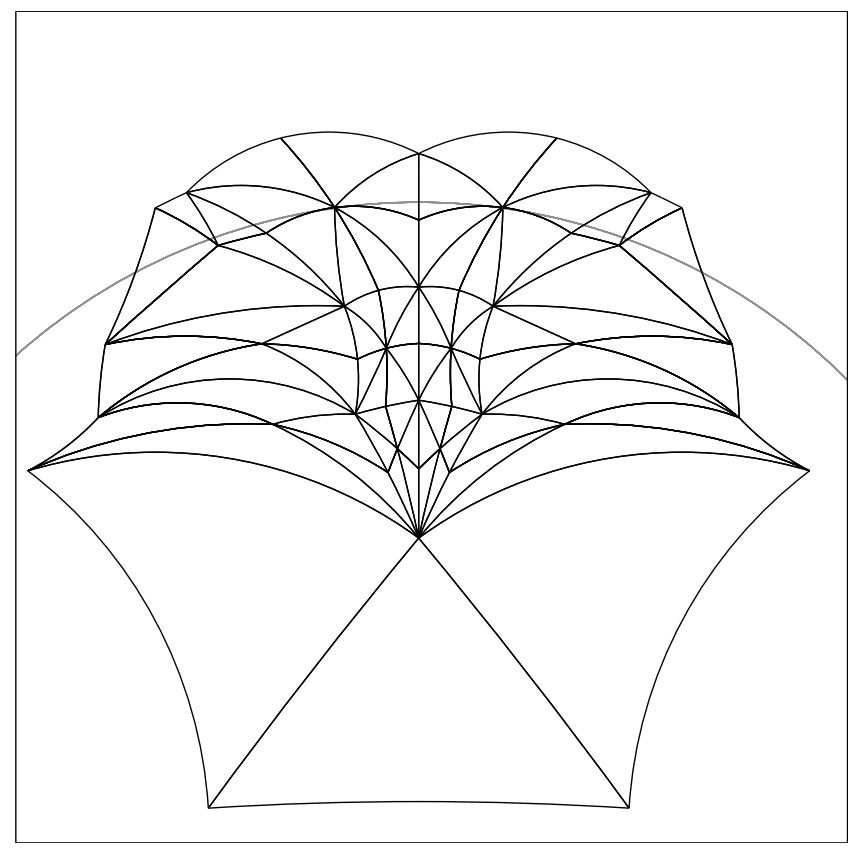

Figure 8.4

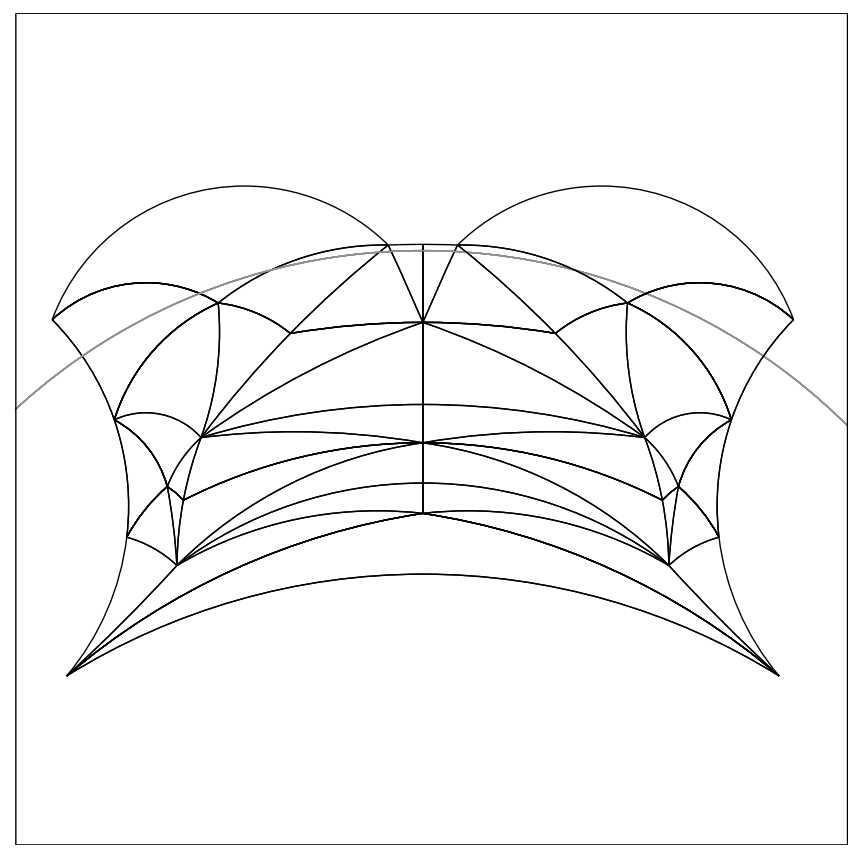

Figure 8.5 


\section{The Computations}

\subsection{The Purity Assumption and Affine Boundedness}

In this section we verify that $Z^{\prime}$ satisfies the Purity Assumption, and make an auxilliary calculation. We are interested in the three subsets: $N_{-} ; N_{+}$; and the affine patch. We first explain crude computational tests which can verify that a simplex is contained in each of these three sets.

Given any simplex $T$, either in $\boldsymbol{C}^{2,1}$ or in $\boldsymbol{C}$, let $\rho(T)$ be the maximum Euclidean distance from the barycenter of $T$ to a vertex of $T . \pi_{j}: \boldsymbol{C}^{2,1} \rightarrow \boldsymbol{C}$ be the $j$ th coordinate projection. Let

$$
V_{j, \pm}(T)=\left|\pi_{j}(\underline{T})\right| \pm\left|\rho\left(\pi_{j}(T)\right)\right| .
$$

For any vector $X \in T$ we have the bounds $V_{j,-}(T) \leq\left|\pi_{j}(X)\right| \leq V_{j,+}(T)$. We define

1. $\tau_{-}(T)=1$ if $V_{1,+}^{2}(T)+V_{2,+}^{2}(T)<V_{3,-}^{2}(T)$. In this case $T$ is negative.

2. $\tau_{+}(T)=1$ if $V_{1,-}^{2}(T)+V_{2,-}^{2}(T)>V_{3,+}^{2}(T)$. In this case $T$ is positive.

3. $\tau_{a}(T)=1$ if $V_{3,-}(T)>0$. In this case $T$ is affinely bounded.

Otherwise we define $\tau_{*}(T)=0$.

Our computational tests do not necessarily work right off the bat. The simplices might be so big as to foil our crude tests. With a view towards refining the tests, we explain how we subdivide simplices in half. If $e$ is an edge we define $e^{\prime}=\left\{e_{1}, e_{2}\right\}$, where $e_{1}$ and $e_{2}$ are the edges obtained by cutting $e$ in half. If $T$ is a higher dimensional simplex, we choose an edge $e$ of $T$ and let $T^{e}$ be the codimension 2 sub-simplex opposite $e$, so that $T=T^{e} \bowtie e$. We define $T^{\prime}=\left\{T_{1}, T_{2}\right\}$ where $T_{j}=T^{e} \bowtie e_{j}$. We can iterate this subdivision process, and when we do so we cycle through the edges in such a way that the diameters of the tetrahedra shrink to points as the process is iterated.

Here is our refined test. Suppose that $\tau$ is any of the three functions defined above. We begin with the singleton list $\{T\}$. In general, suppose we have a list of simplices, and $S$ is the last member of the list. We compute $\tau(S)$. If $\tau(S)=1$ we delete $S$ from the list and repeat. If $\tau(S)=0$ we delete $S$ from the list and append to the list the two tetrahedra of the subdivision of $S$. If the list becomes empty we have succeeded. Call this the subdivision algorithm. 
Let $T_{1}, \ldots, T_{N}$ be the list of the $N=393$ tetrahedra in the union of pieces $A^{\prime}(0) \cup B^{\prime}(0) \cup A^{\prime}(1) \cup B^{\prime}(1)$. An arbitrary tetrahedron in $Z^{\prime}$ has the form $K^{a}\left(T_{b}\right)$ for some $b \in\{1, \ldots, N\}$. It suffices to verify the Purity Assumption for $T_{1}, \ldots, T_{N}$. For each $j$, we compute the types of the vertices of $T_{j}$ and thereby identify $T_{+}$and $T_{-}$, the maximal negative and positive simplices of $T$. We then apply the subdivision algorithm, using $\tau_{-}$(respectively $\tau_{+}$), to verify that $T_{-}$(respectively $T_{+}$) is negative (respectively positive). Using this method, we prove

Lemma 9.1 (Computation 1) $T_{j}$ satisfies the Purity Assumption for all $j=1, \ldots, N$.

For later use, we also compute

Lemma 9.2 (Computation 2) $K^{i}\left(T_{j}\right)$ is affinely bounded for all $|i| \leq 5$ and for all $j=1, \ldots, N$.

\subsection{Clarifying the Assumptions}

In order to deal with the Combinatorial Assumption and the Homeomorphism Assumption efficiently we first need to clarify them.

We can assign a combinatorial code to each vertex of $Z^{\prime}$. First of all $\bullet m$ is coded as $(0,1, m) \in \boldsymbol{Z}^{3}$ and on is coded as $(0,2, n)$. There are three vertices on the edge $[\bullet m \circ n]$; these are coded as $(j, m, n)$ for $j=1,2,3$. Any other vertex of $Z^{\prime}$ not already coded is the obtained as the barycenter of a finite number of points already coded. We code the barycenter of $k$ coded points $\left(a_{i}, b_{i}, c_{i}\right)$ as the union $\bigcup\left(a_{i}, b_{i}, c_{i}\right)$. Two codes agree iff they are the same subset of $\boldsymbol{Z}^{3}$.

We build an abstract simplicial complex $\widetilde{Z}^{\prime}$, as follows. Each tetrahedron of $Z^{\prime}$ defines a 4 -tuple of subsets of $\boldsymbol{Z}^{3}$. We take one tetrahedron for each such 4-tuple, and glue two tetrahedra together along the convex hull of their common vertices. We have a map $\widetilde{Z}^{\prime} \rightarrow Z^{\prime}$. Each coded point is mapped to the point in $\boldsymbol{C}^{2,1}$ it represents and the tetrahedra are mapped in linearly. The Combinatorial Assumption says that the map $\widetilde{Z}^{\prime} \rightarrow Z^{\prime}$ is a homeomorphism. The Homeomorphism Assumption says that the map $Z^{\prime} \rightarrow\left[Z^{\prime}\right]$ is a homeomorphism. We will prove that the composition $\widetilde{Z}^{\prime} \rightarrow\left[Z^{\prime}\right]$ is a homeomorphism, which establishes both assumptions at the same time. 


\subsection{The Vertices}

In this section we show that the map $\widetilde{Z}^{\prime} \rightarrow\left[Z^{\prime}\right]$ is a homeomorphism when restricted to the vertices. Our method in this baby case serves as the model for what we do in general.

We define

$$
C \widetilde{Z}^{\prime}=\bigcup_{a=-4}^{4} \bigcup_{b=1}^{N} K^{a}\left(T_{b}\right) .
$$

We call $C \widetilde{Z}^{\prime}$ the computational portion of $\widetilde{Z}^{\prime}$. it contains $9 \times 393=3537$ tetrahedra. By a direct computation we show

Lemma 9.3 (Computation 3) Suppose $v_{1}$ and $v_{2}$ are two combinatorially distinct vertices of $C \widetilde{Z}^{\prime}$. Then $\left[v_{1}\right] \neq\left[v_{2}\right]$.

By symmetry, Lemma 9.3 takes care of all vertices $v_{1} \in K^{a}\left(T_{b}\right)$ and $v_{2} \in K^{c}\left(T_{d}\right)$ when $|c-a| \leq 8$. We now establish a single estimate which takes care of all remaining pairs of vertices.

Recall that $E_{0} K$ and $E_{\infty} K$ are the null eigenvectors of $K$, normalized so that their third coordinates are 1 . Recall also that $E_{+} K=E_{0} K \otimes E_{\infty} K$ is a positive eigenvector of $K$. Let $E K \subset C^{2,1}$ be the $C$-linear span of $E_{0} K$ and $E_{\infty} K$. Note that $E K$ consists of those vectors which are $\langle$,$\rangle -orthogonal$ to $E_{+} K$.

We map $E K$ into $C^{1,1}$ using the linear map

$$
\Pi_{1}\left(z E_{\infty} K+w E_{0} K\right)=(z, w)
$$

We define the projection map $\Pi_{K}: C^{2,1} \rightarrow E K$

$$
\Pi_{2}(V)=V-\frac{\left\langle V, E_{+} K\right\rangle}{\left\langle E_{+} K, E_{+} K\right\rangle} E_{+} K .
$$

We define the composition

$$
\Pi_{K}=\Pi_{1} \circ \Pi_{2}: \boldsymbol{C}^{2,1} \rightarrow \boldsymbol{C}^{1,1}
$$

and the projectivization of this composition

$$
\pi_{K}(v)=\left[\Pi_{K}(v)\right]
$$


Lemma 9.4 If $\left|\pi_{K}\left(v_{1}\right)\right|<1$ then $\left|\pi_{K}\left(K^{-1}\left(v_{1}\right)\right)\right|<1$. If $\left|\pi_{K}\left(v_{2}\right)\right|>1$ then $\left|\pi_{K}\left(K\left(v_{2}\right)\right)\right|>1$.

Proof: The action of $\Pi_{2} \circ K \circ \Pi_{2}^{-1}$ has eigenvectors $(1,0)$ and $(0,1)$. Hence the projective action of $\Pi_{2} \circ K \circ \Pi_{2}^{-1}$ on $C$ is simply a map of the form $z \rightarrow \mu z$ for some constant. We check that $|\mu|>1$. This immediately implies our lemma.

We do another direct computation to show that

Lemma 9.5 (Computation 4) $\left|\pi_{K}\left(v_{1}\right)\right|<1$ if $v_{1} \in K^{-5}\left(T_{b}\right)$ and $\left|\pi_{K}\left(v_{2}\right)\right|>1$ if $v_{2} \in K^{4}\left(K_{d}\right)$.

Combining the two previous results we see that $\pi_{K}\left(v_{1}\right) \neq \pi_{K}\left(v_{2}\right)$ provided that $v_{1} \in K^{a}\left(T_{b}\right)$ and $v_{2} \in K^{c}\left(T_{d}\right)$ and $|c-a| \geq 9$. But this implies that $\left[v_{1}\right] \neq\left[v_{2}\right]$. We now know that the map $\widetilde{Z}^{\prime} \rightarrow\left[Z^{\prime}\right]$ is a homeomorphism when restricted to the vertices.

\subsection{Reduction to a Finite Computation}

Our problem is to show that the map $\widetilde{Z}^{\prime} \rightarrow\left[Z^{\prime}\right]$ is a homeomorphism. It clearly suffices to show that the map is an injection. At the end of the chapter we prove

Lemma 9.6 $\left[K^{a}\left(T_{b}\right)\right] \cap\left[K^{c}\left(T_{d}\right)\right]=\emptyset$ provided that $|a-c| \geq 9$.

Lemma 9.6 reduces our problem to the checking of tetrahedra within the computational portion of $\widetilde{Z}^{\prime}$. Before we state exactly what needs to be done we record a simple but powerful technical result.

Lemma 9.7 Suppose $[X]=[Y]$ then the line $\overline{X Y}$ is a single point.

Proof: If $[X]=[Y]$ then $X$ and $Y$ are contained in the same 1-dimensional complex linear subspace of $C^{2,1}$. But then $\overline{X Y}$ is also contained in this subspace. Hence, all points on $\overline{X Y}$ are projectively equivalent.

Now we get down to business. Say that a pair $\left(K^{a}\left(T_{b}\right), K^{c}\left(T_{d}\right)\right)$ of tetrahedra is a computational pair if

$$
|a| \leq 5 ; \quad|c| \leq 4 ; \quad a+c \in\{-1,0\} .
$$


Every pair of tetrahedra in $C \widetilde{Z}^{\prime}$ is equivalent to a computational pair under the action of $J$ and $K$.

Given two simplices $T$ and $T^{\prime}$ in $\widetilde{Z}^{\prime}$ we let $T \# T^{\prime}$ denote the number of vertices common to $T$ and $T^{\prime}$. Let $T \square T^{\prime}$ denote the convex hull of these common vertices. Let $T \searrow T^{\prime}=T-\left(T \square T^{\prime}\right)$.

Lemma 9.8 Suppose that $\left[T \searrow T^{\prime}\right] \cap\left[T^{\prime}\right]=\emptyset$ and $\left[T^{\prime} \searrow T\right] \cap[T]=\emptyset$ for every computational pair $\left(T, T^{\prime}\right)$. Then the map $\widetilde{Z}^{\prime} \rightarrow\left[Z^{\prime}\right]$ is an injection.

Proof: Suppose $x, x^{\prime} \in \widetilde{Z}^{\prime}$ are two distinct points such that $[x]=\left[x^{\prime}\right]$. There are two tetrahedra $T$ and $T^{\prime}$ such that $x \in T$ and $x^{\prime} \in T^{\prime}$. If there are several choices for these tetrahedra, we choose so as to minimize $T \# T^{\prime}$. By Lemma 9.6 we can assume that $\left(T, T^{\prime}\right)$ is a computational pair.

Suppose that $T \# T^{\prime}=4$. That is, $T=T^{\prime}$. Let $I=\overline{x x^{\prime}} \cap T$. By Lemma 9.7 the image $[I]$ is a single point. If we want to get a contradiction in this case, it suffices to consider the case when $x$ and $x^{\prime}$ are the endpoints of $I$. Hence $x, x^{\prime} \in \partial T$. Both endpoints of $I$ cannot be in the interior of a single face. Hence, at least one of $x$ or $x^{\prime}$, say $x$, is not contained in the interior of a pure positive face. But all other faces of $Z^{\prime}$ are contained in two tetrahedra. Thus, there is a tetrahedron $T^{\prime \prime} \neq T$ such that $x \in T^{\prime \prime}$. In this way we reduce the case $T \# T^{\prime}=4$ to one of the cases when $T \# T^{\prime} \leq 3$.

Suppose that $T \# T=3$. In this case the hypotheses imply that $x$ and $x^{\prime}$ are both contained in the triangle $T \square T^{\prime}$. The same kind of argument as in the previous case shows that we can assume that $x$ and $x^{\prime}$ are contained in the boundary of $T \square T^{\prime}$. If $x$ and $x^{\prime}$ are contained in the same edge of $T \square T^{\prime}$ then this entire edge is mapped to a point, by Lemma 9.7. This contradicts the fact that [ ] is injective on the vertices of $Z$. If $x$ and $x^{\prime}$ are contained in different edges then we can find tetrahedra $S$ and $S^{\prime}$ such that $x \in S$ and $x^{\prime} \in S^{\prime}$ and $S \# S^{\prime} \leq 2$. This contradicts the our choice of $T$ and $T^{\prime}$.

If $T \# T^{\prime}=2$ then our hypotheses imply that $x$ and $x^{\prime}$ are contained in the same edge of $Z$. This gives the same contradiction as in the previous case. If $T \# T^{\prime}=1$ then our hypotheses imply that $x=x^{\prime}$. If $T \# T^{\prime}=0$ we contradict our hypotheses immediately.

\subsection{The Pseudorandom Projection Algorithm}

Suppose that $S$ and $S^{\prime}$ are two line segments in $\boldsymbol{C}^{1,1}$. The projective images $[S]$ and $\left[S^{\prime}\right]$ are (generically) arcs of circles in $\boldsymbol{C}$. We let $I\left(S, S^{\prime}\right)$ denote the 
number of points in $[S] \cap\left[S^{\prime}\right]$. In $\S 10.4$ we will explain how we compute $I\left(S, S^{\prime}\right)$.

Suppose that $U$ is a tetrahedron in $C^{1,1}$. (In practice $U$ is the image of a tetrahedron in $\boldsymbol{C}^{2,1}$ under a linear projection.) Let $e_{1}, \ldots, e_{6}$ be the edges of $U$. The images $\left[e_{i}\right]$ is a (possibly degenerate) arc of a circle in $\boldsymbol{C} \cup \infty$. Let $\underline{U}$ be the barycenter of $U$. Let $\langle U\rangle$ be the closure of the union of the bounded components of $\boldsymbol{C}-\bigcup\left[e_{j}\right]$.

Lemma 9.9 If $U$ is affinely bounded-that is, contained in the affine patch defined in $\S 2-$ then $[U] \subset\langle U\rangle$.

Proof: First we claim that $[U] \subset[\partial U]$. Let $x \in T$. The smooth map $U \rightarrow[U]$ has 3 -dimensional domain and 2-dimensional range. Hence [] is not injective in any neighborhood of $x$. There are sequences $\left\{a_{m}\right\}$ and $\left\{b_{m}\right\}$ such that $a_{m} \rightarrow x$ and $b_{m} \rightarrow x$ and $a_{m} \neq b_{m}$ and $\left[a_{m}\right]=\left[b_{m}\right]$. The line $\overline{a_{m} b_{m}}$ intersects $\partial U$ in at least one point, which we call $c_{m}$. The same argument as in Lemma 9.7 says that $\left[c_{m}\right]=\left[a_{m}\right]$. Since $\partial U$ is compact, we can assume that $\left\{c_{m}\right\} \rightarrow y \in \partial U$. We have $[y]=[x]$ by continuity. This shows that $[U] \subset[\partial U]$. Next, we claim that $[\sigma] \subset\langle U\rangle$, when $\sigma$ is a face of $U$. Otherwise, the boundary of $[\sigma]$ contains a point $p=[x] \notin\langle U\rangle$. In this case [ ] is not injective in any neighborhood of $x$ and the same argument as in the first step shows that $[x] \in L(\partial \sigma) \subset \bigcup\left[e_{j}\right]$. This is a contradiction.

Corollary 9.10 (Projection Test) If $U$ and $U^{\prime}$ are affinely bounded tetrahedra of $\boldsymbol{C}^{1,1}$ and $^{1}$

1. $U \# U^{\prime} \leq 2$.

2. $\left[e_{i}\right] \cap\left[e_{j}^{\prime}\right]=\left[e_{i} \square e_{j}^{\prime}\right]$ for all $i, j$.

3. $[\underline{T}] \notin\left\langle U^{\prime}\right\rangle$ and $\left[\underline{T}^{\prime}\right] \notin\langle U\rangle$.

Then $\left[T-T \square T^{\prime}\right] \cap\left[T^{\prime}\right]=\emptyset$.

\footnotetext{
${ }^{1}$ Even though we originally defined the listed quantities for tetrahedra in $\boldsymbol{C}^{2,1}$ we make the same definitions in $\boldsymbol{C}^{1,1}$.
} 
Proof: There are three cases.

Case 0: Suppose that $U \# U^{\prime}=0$. Then $\left[e_{i}\right] \square\left[e_{j}^{\prime}\right]=\emptyset$ for all $i, j$. Hypothesis 2 says that $\left[e_{i}\right]$ and $\left[e_{j}^{\prime}\right]$ are disjoint for all $i, j$. This means that $\langle U\rangle$ and $\left\langle U^{\prime}\right\rangle$ have disjoint boundaries. $\langle U\rangle$ cannot be contained in the interior of $\left\langle U^{\prime}\right\rangle$ because $[\underline{T}] \notin\left\langle U^{\prime}\right\rangle$. Likewise $\left\langle U^{\prime}\right\rangle$ cannot be contained in the interior of $\langle U\rangle$. The only possibility is that $\langle U\rangle \cap\left\langle U^{\prime}\right\rangle=\emptyset$. Lemma 9.9 now applies.

Case 1: Suppose that $U \# U^{\prime}=1$ and, for the sake of contradiction, that there is some $y \in U-x$ such that $[y] \in\left[U^{\prime}\right]$. From Lemma 9.9 we have $[U] \in\langle U\rangle$ and $\left[U^{\prime}\right] \in\left\langle U^{\prime}\right\rangle$. By Hypothesis 2, we have $\partial\langle U\rangle \cap \partial\left\langle U^{\prime}\right\rangle=[x]$. Given Hypothesis 3, we must have $\langle U\rangle \cap\left\langle U^{\prime}\right\rangle=[x]$. But this forces $[y]=[x]$. Let $\sigma$ be the face of $U$ opposite $x$. The point $z=\overline{x y} \cap \sigma$ exists and the same argument as in Lemma 9.7 says that $[z]=[x]$. Therefore $[x] \in[\sigma]$. From Hypothesis 2, we have $x \in[\sigma]-[\partial \sigma]$. The same argument as in Lemma 9.9 shows that $[\sigma] \subset\langle\sigma\rangle$. Thus $[x]$ is contained in the interior of $\langle\sigma\rangle \subset\langle U\rangle$. This contradicts the fact that $\langle U\rangle \cap\left\langle U^{\prime}\right\rangle=[x]$.

Case 2: Suppose that $U \# U^{\prime}=2$ so that $U \square U^{\prime}=e$, a common edge. Let $e_{1}=e$ and let $e_{2}, \ldots, e_{6}$ be the other edges of $U$. Suppose there is some $y \in U-e_{1}$ such that $[y] \in\left[U^{\prime}\right]$. An argument similar to the one given in the previous case show that $\langle U\rangle \cap\left\langle U^{\prime}\right\rangle=\left[e_{1}\right]$ and that $[y] \in\left[e_{1}\right]$. There is some point $x \in e_{1}$ such that $[y]=[x]$. The line $\overline{x y}$ intersects $\partial T$ in a point $z_{1} \notin e_{1}$, and $\left[z_{1}\right]=[x]$. The line $\overline{x z_{1}}$ intersects $e_{2} \cup \ldots \cup e_{6}$ in a point $z_{2}$, and $\left[z_{2}\right]=[x]$. If $z_{2}$ lies on $\left[e_{j}\right]$ than $\left[e_{j}\right] \cap\left[e_{1}^{\prime}\right]$ has too many intersections, contradicting Hypothesis 2. This contradiction finishes the proof.

We use the technique in $\S 10.4$ to verify Hypothesis 2 . To verify Hypothesis 3 , we let $S \subset C^{1,1}$ be the line segment with endpoints $\underline{U^{\prime}}$ and $[1,0]$. Note that $\left[S^{\prime}\right]$ is an infinite ray emanating from $\left[\underline{U}^{\prime}\right]$. The graph $\langle U\rangle$ has 4 length-3 circuits, one corresponding to each face of $U$. For each of these circuits $\sigma$ we compute $\sum_{e \in \sigma} I\left(S^{\prime}, e\right)$. If all 4 sums are even then there our ray intersects each length 3 circuit of $U$ an even number of times. It follows from a degree argument that the endpoint of the ray, namely $\left[\underline{U}^{\prime}\right]$, cannot belong to $\langle U\rangle$. To verify that $[\underline{U}] \notin\langle U\rangle^{\prime}$ we make the same computations, with the roles of $U$ and $U^{\prime}$ reversed.

The Projection Test is useful to us when we have a rich supply of projections at our disposal. We now define these projections. Given any $r \in[0, \infty)$ 
let $d(r) \in[-1,1)$ be twice the decimal part of $r$, minus 1. Given primes $p, q$ we define

$$
z(p, q ; n)=d(n \sqrt{p})+i d(n \sqrt{q}) .
$$

Given a vector $V=\left(V_{1}, V_{2}, V_{3}\right) \in \boldsymbol{C}^{2,1}$, and $n \in \boldsymbol{N}$, we define

$$
\operatorname{Proj}_{n}(V)=\left(z(2,3 ; n) V_{1}+z(5,7 ; n) V_{2}, V_{3}\right) \in \boldsymbol{C}^{1,1} .
$$

$\operatorname{Proj}_{n}$ is a pseudorandom projection from $\boldsymbol{C}^{2,1}$ into $\boldsymbol{C}^{1,1}$ which carries the affine patch of $\boldsymbol{C}^{2,1}$ to the affine patch of $\boldsymbol{C}^{1,1}$. If $\left(T, T^{\prime}\right)$ is a computational pair of tetrahedra we define $U_{n}=\operatorname{Proj}_{n}(T)$ and $U_{n}^{\prime}=\operatorname{Proj}_{n}\left(T^{\prime}\right)$. From Computation 2, we know that $U_{n}$ and $U_{n}^{\prime}$ are affinely bounded for all $n$. When $T \# T^{\prime} \leq 2$ we attempt to verify the hypotheses of the Projection Test for $n=1,2,3 \ldots$ until we meet with success or have 10000 failures in a row. We call this the Pseudorandom Projection Algorithm.

Using the Pseudorandom Projection Algorithm we prove

Lemma 9.11 (Computation 5) Suppose $\left(T, T^{\prime}\right)$ is a computational pair and $T \# T^{\prime} \leq 1$. Then $\left[T \searrow T^{\prime}\right] \cap\left[T^{\prime}\right]=\emptyset$ and $\left[T^{\prime} \searrow T\right] \cap[T]=\emptyset$.

We know from Computation 4 that $T \# T^{\prime}=0$ if $T=K^{-5}\left(T_{b}\right)$ and $T^{\prime}=$ $K^{5}\left(T_{d}\right)$. Therefore, we have a technical result which comes in handy in the next section:

Corollary 9.12 For each $j=1, \ldots, N$ we have

$$
\left[K^{-5}\left(T_{j}\right)\right] \cap\left[K^{4}\left(T_{j}\right)\right]=\emptyset .
$$

When we apply the Pseudorandom Projection Algorithm to the pairs $\left(T, T^{\prime}\right)$ such that $T \# T^{\prime}=2$ we have about 4400 successes and 40 failures. In the next section we introduce a new computational test which deals with these failures.

\subsection{The Edge Smash Test}

Suppose that $\left(T, T^{\prime}\right)$ is a computational pair of tetrahdedra such that $T \# T^{\prime}=$ 2 . Let $v_{1}$ and $v_{2}$ be the common vertices. Let $e$ be the common edge. Let $\vec{e}=v_{1}-v_{2}$. In other words $\vec{e}$ is just $e$, but interpreted as a vector. Let

$$
T_{e}=\operatorname{Proj}_{1}(T \otimes \vec{e}) ; \quad T_{e}^{\prime}=\operatorname{Proj}_{1}\left(T^{\prime} \otimes \vec{e}\right) .
$$


Here $\otimes$ is as in Equation 8 and $\operatorname{Proj}_{1}$ is as in Equation 105. Essentially we are "smashing" the edge $e$ to a single point and then taking a generic projection into $\boldsymbol{C}^{1,1}$.

The map $v \rightarrow \operatorname{Proj}_{1}(v \otimes \vec{e})$ is complex linear. Moreover $v_{1} \otimes \vec{e}=$ $v_{2} \otimes \vec{e}$. From these two properties it follows that (generically) $T_{e}$ is a triangle. Generically $\left[\partial T_{e}\right]$ is a union of 3 circular arcs, joined in a cyclic fashion. Let $e_{1}, e_{2}, e_{3}$ be the three edges of $T_{e}$ and let $e_{1}^{\prime}, e_{2}^{\prime}$ and $e_{3}^{\prime}$ be the three edges of $T_{e}^{\prime}$.

\section{Lemma 9.13 (Edge Smash Test) Suppose that $T \# T \geq 2$ and}

1. $\left[e_{i}\right] \cap\left[e_{j}^{\prime}\right]=\left[e_{i} \square e_{j}^{\prime}\right]$ for all $i, j$.

2. $\beta$ intersects $\left[\partial T_{e}\right]$ and $\left[\partial T_{e}^{\prime}\right]$ each an odd number of times.

Then $\left[T-T \square T^{\prime}\right] \cap\left[T^{\prime}\right]=\emptyset$ and $\left[T^{\prime}-T \square T^{\prime}\right] \cap[T]=\emptyset$

Proof: We consider the case $T \# T^{\prime}=2$. The case $T \# T^{\prime}=3$ is similar. In general we write $S_{e}=\operatorname{Proj}_{1}(S \otimes \vec{e})$ for any subset $S \subset C^{2,1}$.

We will establish the first conclusion. The second one has the same proof, with the roles of $T$ and $T^{\prime}$ interchanged. Essentially the same argument as in Lemma 9.9 shows that

$$
\left[T_{e}\right] \cap\left[T_{e}^{\prime}\right]=\left[e_{e}\right]
$$

Suppose $x \in T-T \square T^{\prime}$ is such that $[x] \in\left[T^{\prime}\right]$. Since $x$ is a multiple of a vector in $T^{\prime}$, the vector $x_{e}$ is a multiple of a vector in $T_{e}^{\prime}$. Hence $\left[x_{e}\right] \in\left[T_{e}^{\prime}\right]$. This means that $\left[x_{e}\right]=\left[e_{e}\right]$. On the other hand, $x_{e} \neq e_{e}$ because $x \notin e$. The line $\overline{x_{e} e_{e}}$ intersects $e_{e}^{\mathrm{op}}$ in a point $z_{e}$. Here $e^{\mathrm{op}}$ is the edge of $T$ opposite $e$. But then $\left[z_{e}\right]=\left[e_{e}\right]$, contradicting the fact that $\left[e_{e}^{\mathrm{op}}\right]$ is disjoint from $\left[T_{e}^{\prime}\right]$.

We verify Hypotheses 1 and 2 using the technique of $\S 10.4$. Given $T$ and $T^{\prime}$ as above we first try to verify the hypotheses of the Edge Smash Test. If the Edge Smash Test fails we then apply the Pseudorandom Projection Algorithm. All but 3 pairs $\left(T, T^{\prime}\right)$, with $T \# T^{\prime}=2$, pass this combined test.

For the remaining three pairs, one of the graphs $\left[T_{e}\right]$ or $\left[T_{e}^{\prime}\right]$, lets say $\left[T_{e}\right]$, is a lune rather than a triangle. Figure 9.1 shows one of the three cases. The problem is that one of the faces $\sigma$ of $T$ is such that $\left[\sigma_{e}\right]$ is a single point 
rather than a nontrivial circular arc. ( $e$ is an edge of $\sigma$.) A straightforward variant of Lemma 9.13 shows, in these degenerate cases, that

$$
\left[T^{\prime} \searrow T\right] \cap[T]=\emptyset ; \quad\left[(T-\sigma) \searrow T^{\prime}\right] \cap\left[T^{\prime}\right]=\emptyset .
$$

We just have to worry that the map $[\sigma] \rightarrow \sigma$ is not injective. If this is the case, then the map $\partial \sigma \rightarrow[\partial \sigma]$ is not injective either. We check explicitly that $\left[\operatorname{Proj}_{1}(\partial \sigma)\right]$ is an embedded triangle. Thus the conclusion to Lemma 9.13 holds in the 3 degenerate cases as well.

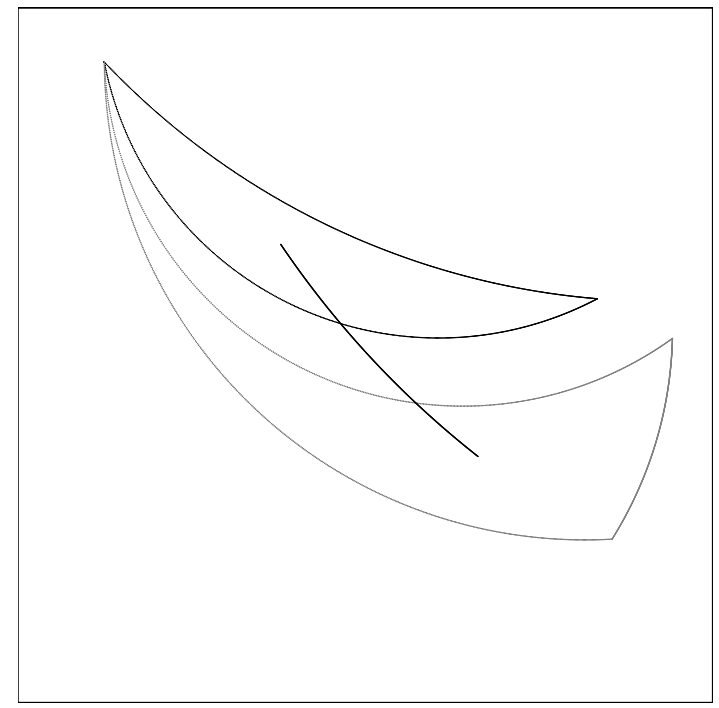

Figure 9.1

We now come to the case when $T \# T^{\prime}=3$. These tetrahedra have 3 edges in common and correspondingly we have three tries at verifying the hypotheses of the Edge Smash Test. We compute that all but 30 pairs pass on at least one try. For the remaining 30 pairs, the graphs $\left[T_{e}\right]$ and $\left[T_{e}^{\prime}\right]$ are lunes which intersect in a single point, for all three edges $e$, as shown in Figure 9.2. This derives from the face that $T \square T^{\prime}$ is contained in a 2-complex-dimensional subspace. 


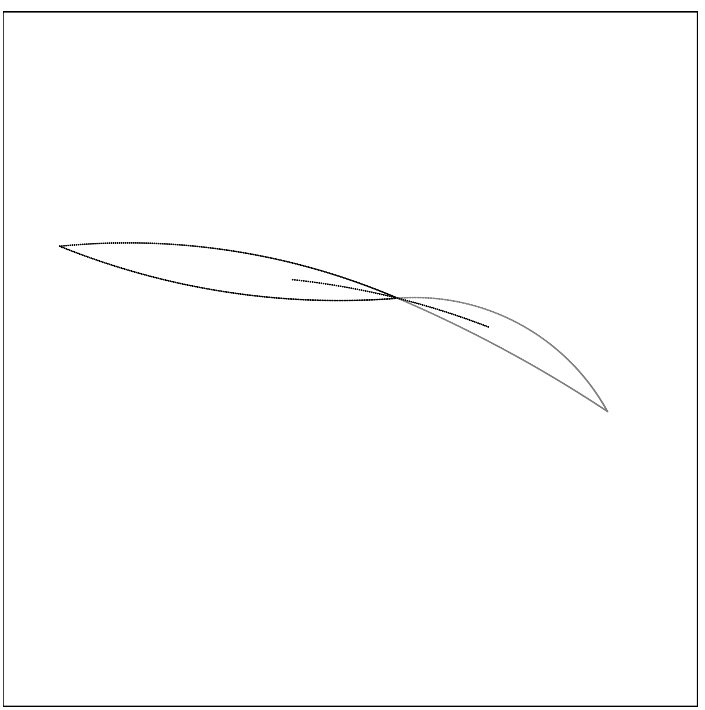

Figure 9.2

A straightforward variant of Lemma 9.13 shows that the same conclusion holds in these degenerate cases. Thus,

Lemma 9.14 (Computation 6) Suppose $\left(T, T^{\prime}\right)$ is a computational pair and $T \# T^{\prime} \geq 2$. Then $\left[T \searrow T^{\prime}\right] \cap\left[T^{\prime}\right]=\emptyset$ and $\left[T^{\prime} \searrow T\right] \cap[T]=\emptyset$.

\subsection{Proof of Lemma 9.6}

Let $E_{K}$ be the map defined in Equation 101.

Lemma 9.15 If $T$ is any tetrahedron of $Z^{\prime}$ then $\left[\Pi_{K}(T)\right]$ exists and is contained in $\boldsymbol{C}-\{0\}$.

Proof: Note that $\Pi_{K}(V)=0$ iff $V$ is a multiple of $E_{+} K$. For the first part of this lemma is suffices to show that $T \cap \boldsymbol{C} E_{+} K=\emptyset$. If this is false then $\left[E_{+} K\right] \in[T]$. But $K$ fixes $\left[E_{+} K\right]$. Setting $T=K^{a}\left(T_{j}\right)$ we see that $\left[E_{+} K\right] \in K^{c}\left(T_{j}\right)$ for all $c$. In particular $\left[K^{-5}\left(T_{j}\right)\right] \cap\left[K^{4}\left(T_{j}\right)\right] \neq \emptyset$. This contradicts Corollary 9.12. Since $T \cap \boldsymbol{C} E_{+} K=\emptyset$, the map $\Pi_{K}$ is defined on $T$. The same argument shows that $T$ is disjoint from $\boldsymbol{C} E_{0} K$ and $\boldsymbol{C} E_{\infty} K$, the sets which projectivize to 0 and $\infty$ respectively. 
Lemma 9.16 If $\left[\Pi_{K}\left(K^{a}\left(T_{b}\right)\right)\right]$ is contained in the interior of the unit disk, then so is $\left[\Pi_{K}\left(K^{a-1}\left(T_{b}\right)\right)\right]$. If $\left[\Pi_{K}\left(K^{a}\left(T_{b}\right)\right)\right]$ is contained in the exterior of the unit disk then so is $\left[\Pi_{K}\left(K^{a+1}\left(T_{b}\right)\right)\right]$.

Proof: Same argument as in Lemma 9.4.

Suppose $T$ is a tetrahedron of $Z^{\prime}$ and we want to prove that $\left[\Pi_{K}(T)\right]$ is contained in the interior of the unit disk. We let $e_{1}, \ldots, e_{6}$ be the 6 edges of $\Pi_{K}(T)$. We let $e_{1}^{\prime}$ be the segment with endpoints $(1,1)$ and $(i,-i)$. Let $e_{2}^{\prime}$ be the segment with endpoints $(1,1)$ and $(-i, i)$. It is not hard to see that $\left[e_{1}^{\prime}\right] \cup\left[e_{2}^{\prime}\right]$ is the unit circle. The same argument as in Lemma 9.9 shows that $\left[\Pi_{K}\right](T)$ is contained in the closure of the union of the bounded components of $\boldsymbol{C}-\bigcup\left[e_{j}\right]$. Hence, if $I\left(e_{i}, e_{j}^{\prime}\right)$ for all 12 pairs of indices we know that $\left[\Pi_{K}(T)\right]$ is disjoint from the unit circle. We then evaluate $\left[\Pi_{K}(\underline{T})\right]$, the image of the barycenter, to determine if our set is in the interior or exterior of the unit disk.

Using this method we prove

Lemma 9.17 (Computation 7) $\left[\Pi_{K}\left(K^{-5}\left(T_{b}\right)\right)\right]$ is contained in the interior of the unit disk and $\left[\Pi_{K}\left(K^{4}\left(T_{b}\right)\right)\right]$ is contained in the exterior of the unit disk.

Lemma 9.6 follows from Lemma 9.16 and Computation 8. This completes our computer-aided proof. 


\section{Some Computational Issues}

\subsection{Interval Arithmetic}

Put in abstract terms, the main computational component of our proof begins with a finite list $r_{1}, \ldots, r_{n}$ of real numbers and establishes a finite list of inequalities of the form $f\left(r_{1}, \ldots, r_{n}\right)>0$. Here $f$ only involves the arithmetic operations and the square root function. The computer performs these functions according to the IEEE standards $[\mathbf{I}]$.

To control round-off error, we implement interval arithmetic, essentially as we described in $[\mathbf{S 1}]$. In brief, we find intervals $I_{1}, \ldots, I_{n}$ such that $r_{j} \in I_{j}$ for all $j$. We perform operations on intervals in a manner which parallels the way they are performed on reals. To control floating point errors, we round outward after computing any interval. For instance, if $I_{1}=\left[a_{1}, b_{1}\right]$ and $I_{2}=\left[a_{2}, b_{2}\right]$ then $I_{1}+I_{2}=\left[c_{1}, c_{2}\right]$, where $c_{1}$ is the rounded-down computation of $a_{1}+b_{1}$ and $c_{2}$ is the rounded-up computation of $a_{2}+b_{2}$. In this way, we have $r_{1}+r_{2} \in I_{1}+I_{2}$. More generally we have $f \in J$, where $J=f\left(I_{1}, \ldots, I_{n}\right)$, the interval version of our expression. To show that $f>0$ it suffices to show that both endpoints of $J$ are positive.

It may happen during the running of our code that certain interval operations are impossible. For instance, if endpoints of $I_{2}$ have opposite signs then we cannot compute $I_{1} / I_{2}$. In any situation like this, we terminate the computation and declare it a failure. Our computations are such that we have many chances to establish each inequality. We don't care about the number of failed attempts, as long as one attempt succeeds.

\subsection{Important Approximate Values}

The numbers $r_{1}, \ldots, r_{n}$, mentioned in the previous section, are the real and imaginary parts of the entries of certain matrices and vectors. For each such $r$, we compute a 16 digit approximation $r^{\prime}$ and store it in an auxilliary file. We replace $r$ by the interval $I_{r}=\left[r^{\prime}-10^{-15}, r^{\prime}+10^{-15}\right]$, so that $r \in I_{r}$.

The approximation $r^{\prime}$ is computed using Mathematica $[\mathbf{W}]$ to evaluate the relevant quantities defined in $\S 3$. We compute the desired quantity to high precision (say 50 decimal places) and then truncate the answer to 16 decimal places. It doesn't matter to us exactly how Mathematica computes these quantities. We check that the computed quantities are close to the actual quantities in an a posteriori manner. That is, we check that that the 
computed quantities satisfy their defining equations to sufficiently high precision. These a posteriori checks only involve the basic arithmetic operations, and long experience tells us that Mathematica can perform basic arithmetic well past our desired precision.

For example, we need to compute an approximation to the matrix $K$, which has the property that $K^{3}=I_{2} I_{1} I_{3}$. We compute $K$ using the equations in $§ 3.2$. We then check that $K^{3}$ agrees to high precision with $I_{2} I_{1} I_{3}$. We also check that $K$, applied to several random vectors, preserves the Hermitian product $\langle$,$\rangle to high precision. Here is the approximation for K$.

$$
\begin{aligned}
& K_{11}=+0.6222788295504330-0.3945932932722004 \sqrt{-1} \\
& K_{12}=-0.0450456615598452-0.7300495221592298 \sqrt{-1} \\
& K_{13}=+0.2254792564437312+0.1646065920488111 \sqrt{-1} \\
& K_{21}=+0.0450456615598452+0.7300495221592298 \sqrt{-1} \\
& K_{22}=-0.7276904827471762-0.5312357878085289 \sqrt{-1} \\
& K_{23}=+0.5389904853208957+0.2371404100806019 \sqrt{-1} \\
& K_{31}=+0.2254792564437312+0.1646065920488111 \sqrt{-1} \\
& K_{32}=-0.5389904853208957-0.2371404100806019 \sqrt{-1} \\
& K_{33}=+1.1933916540738774+0.0223361272114748 \sqrt{-1}
\end{aligned}
$$

We need to compute quantities of the form $K^{n}(v)$, where $|n| \leq 10$ and $v$ is one of a finite list of vectors. In principle we make such a computation by performing $n$ actions of the matrix $K$ on the vector $v$. That is, we compute $K(v)$, then $K(K(v))$, and so on. In practice, we reduce the round-off error (or, rather, the size of the intervals produced by the computation) as follows. We store 16-digit approximations to $K^{3}=I_{2} I_{1} I_{3}$ and $K^{-3}=I_{3} I_{1} I_{2}$ and then compute $K^{3}(v)$ in place of $K(K(K(v)))$ wherever possible. (Likewise for $K^{-3}$.) For instance, if we want to compute $K^{10}(v)$ we actually compute $K^{3}\left(K^{3}\left(K^{3}(K(v))\right)\right)$.

The null eigenvectors of $K$ are $E_{\infty} K$ and $E_{0} K$. These vectors projectivize to give the two fixed points, on $S^{3}$, of the elements $I_{2} I_{1} I_{3}$ and $I_{3} I_{1} I_{2}$, and are also eigenvectors for these elements. We use the Mathematica Eigenvectors[] command to compute the eigenvectors of $I_{2} I_{1} I_{3}$ to high precision. As a check we see that our computed $E_{0} K$ is null to high precision and nearly fixed by the projective actions of $I_{2} I_{1} I_{3}$ and $I_{3} I_{1} I_{2}$. One of these two elements acts quite expansively about the fixed point which is close to our approximation. This fact guarantees that our approximation is extremely close to the actual 
fixed point, for otherwise it would be moved far away from itself by the expansive action. Here is the 16 digit truncation of $E_{0} K$.

$$
\begin{aligned}
& 0.5769388548583972-0.5538518076110999 \sqrt{-1} \\
& 0.5416441550538012+0.2588654906638812 \sqrt{-1} \\
& 1.0000000000000000+0.0000000000000000 \sqrt{-1}
\end{aligned}
$$

To get $E_{\infty} K$ we use $E_{\infty} K=J\left(E_{0} K\right)$, where $J$ is in Equation 19 .

The remaining quantities we list are computed in much the same way as $E_{0} K$.

The lift of (12) has 16 digit approximation

$$
\begin{aligned}
& 0.3700644371104598-0.0115545886073811 \sqrt{-1} \\
& 0.0000000000000000+0.0000000000000000 \sqrt{-1} \\
& 1.0658206932269412-0.0332783115708986 \sqrt{-1}
\end{aligned}
$$

The lift of (121312) has 16 digit approximation

$$
\begin{aligned}
& 0.6322009573071889-1.5008461387048185 \sqrt{-1} \\
& 0.0000000000000000+0.0000000000000000 \sqrt{-1} \\
& 1.7123304263886281-0.8486116269230751 \sqrt{-1}
\end{aligned}
$$

The lift of (1213121312) has 16 digit approximation

$$
\begin{array}{r}
-0.6353698402242000-2.1831056534717000 \sqrt{-1} \\
0.0000000000000000+0.0000000000000000 \sqrt{-1} \\
1.5937497983832000-1.2765604992479000 \sqrt{-1}
\end{array}
$$

The lift of (12131213121312) has 16 digit approximation

$$
\begin{aligned}
& 0.0000000000000000-1.8479830746078751 \sqrt{-1} \\
& 0.0000000000000000+0.0000000000000000 \sqrt{-1} \\
& 1.5540403611351847+0.0000000000000000 \sqrt{-1}
\end{aligned}
$$

\subsection{The Pictures from Chapter 5}

Since we ultimately project our picture of $Z_{0}^{*}$ into the plane, and since the formula for the action on the Moebius group on $\boldsymbol{H}^{3}$ is a bit complicated, 
we do all our calculations in the plane. We never actually need to consider points in $\boldsymbol{H}^{3}$ at all.

We represent a point in $\boldsymbol{H}^{3}$ as the intersection of two geodesics. This is natural from our point of view: The special points $\alpha_{n}^{0}$ and $\beta_{2 m}^{0}$ are the intersection points of the axes of two halfturns. We store the geodesics as pairs of complex numbers-namely, the endpoints of the geodesics. To recover

a point in $\boldsymbol{H}^{3}$ from the pair of geodesics, we find the planar intersection of the two line segments bounded by the endpoints of the geodesics, and then adjust the third coordinate, using the Pythagorean theorem. (Actually, we never need to find that third coordinate.) If we want to figure out the action of an element of $\mathrm{PSL}_{2}(\boldsymbol{C})$ on one of our points, we move the endpoints of the geodesics and then recompute the intersection.

We frequently need to find the midpoint of a geodesic segment in $\boldsymbol{H}^{3}$. We will describe the situation when $\gamma$ is the geodesic with endpoints -1 and +1 . The general case is similar. Let $\alpha$ and $\beta$ be two points on $\gamma$. We assume that the points are ordered as $-1, \alpha, \beta, 1$. Let $a$ and $b$ be the corresponding projections into the plane. We have $-1<a<b<1$. Let $\xi$ be the point on $\gamma$ halfway between $\alpha$ and $\beta$. Let $x$ be the projection into the plane. The formula for $x$ is:

$$
x=\frac{d_{1}-d_{2}}{d_{2}+d_{2}} ; \quad d_{1}=(1+b) \sqrt{1-a^{2}} ; \quad d_{2}=(1-a) \sqrt{1-b^{2}} .
$$

One can verify this formula by showing that the cross ratio of the points $-1, a^{\prime}, x^{\prime}, 1$ is the same as the cross ratio of the points $-1, x^{\prime}, b^{\prime}, 1$. Here $a^{\prime}=a+\sqrt{a^{2}-1}$, etc.

Let us explain how we find, say, the point $\alpha_{0}^{+}$. First we take the point on $\alpha_{0}$ which is halfway between $A_{10}^{*}\left(\alpha_{13}^{-}\right)$and $\alpha_{0}^{+}$. Call this point $\zeta$. Next, we take the point on $\alpha_{0}$ which is halfway between $\zeta$ and $A_{10}^{*}\left(\alpha_{13}^{0}\right)$. This gives the point which is $3 / 4$ of the way from $\alpha_{0}^{0}$ to $A_{10}^{*}\left(\alpha_{13}^{0}\right)$, as desired. (Compare equation 66.) We emphasize that all these calculations are done from plane geometry. The points $\alpha_{0}^{-}$and $\alpha_{1}^{ \pm}$and $\beta_{0}^{ \pm}$are computed similarly. Once we have these 6 points, we get the remaining (plotted) vertices of $Z_{0}^{*}$ using the action of $K_{*}$.

\subsection{Intersections of Circular Arcs}

We now explain how to compute $I\left(S, S^{\prime}\right)$, the intersection number of two circular arcs $[S]$ and $\left[S^{\prime}\right]$. Here $S$ and $S^{\prime}$ are segments in $\boldsymbol{C}^{1,1}$. For ease of 
exposition we will assume that $[S]$ and $\left[S^{\prime}\right]$ only intersect at interior points. The other cases are handled by similar methods. Our method does not work on conceivable pair of segments. The code is written in such a way that it either returns a guaranteed correct value for $I\left(S, S^{\prime}\right)$, or it indicates that the method fails. To rule out gross programming errors, we wrote a Tcl program which allows us to specify two circular arcs graphically, compute their intersection number, then check the result with our eyes.

We write $S=\left(S_{1}, S_{2}\right)$ Here $S_{1}, S_{2} \subset C^{1,1}$ are the endpoints of $S$. We write $S_{1}=\left(S_{11}, S_{12}\right)$ and $S_{2}=\left(S_{21}, S_{22}\right)$. We make the same definitions for $S^{\prime}$. The matrix

$$
T_{S}=\left[\begin{array}{cc}
S_{22} & -S_{21} \\
-S_{12} & S_{11}
\end{array}\right]
$$

has the property that the endpoints of $T_{S}(S)$ have the form $(z, 0)$ and $(0, z)$ for some $z \in \boldsymbol{C}$. Thus $\left[T_{S}(S)\right]=[0, \infty]$. We write $S^{\prime \prime}=T_{S}\left(S^{\prime}\right)$. Clearly $I\left(T_{S}(S), T_{S}\left(S^{\prime}\right)\right)=I\left(S, S^{\prime}\right)$. Each interior point on $S^{\prime \prime}$ is projectively equivalent to $t S_{1}^{\prime \prime}+S_{2}^{\prime \prime}$ for some $t>0$. Such a point projects to $(0, \infty)$ iff

$$
\left(t S_{11}^{\prime \prime}+S_{21}^{\prime \prime}\right)\left(t \bar{S}_{12}^{\prime \prime}+\bar{S}_{22}^{\prime \prime}\right) \in(0, \infty)
$$

Thus $t$ must satisfy the relations

$$
\begin{aligned}
& P(t)=a_{0} t^{2}+b_{0} t+c_{0}=0 ; \quad t>0 ; \quad a_{1} t^{2}+b_{1} t+c_{1}>0 . \\
& a_{0}=\Im\left(S_{11}^{\prime \prime} \bar{S}_{12}^{\prime \prime}\right) ; \quad b_{0}=\Im\left(S_{11}^{\prime \prime} \bar{S}_{22}^{\prime \prime}+S_{21}^{\prime \prime} \bar{S}_{12}^{\prime \prime}\right) ; c_{0}=\Im\left(S_{21}^{\prime \prime} \bar{S}_{22}^{\prime \prime}\right) . \\
& a_{1}=\Re\left(S_{11}^{\prime \prime} \bar{S}_{12}^{\prime \prime}\right) ; \quad b_{1}=\Re\left(S_{11}^{\prime \prime} \bar{S}_{22}^{\prime \prime}+S_{21}^{\prime \prime} \bar{S}_{12}^{\prime \prime}\right) ; \quad c_{1}=\Re\left(S_{21}^{\prime \prime} \bar{S}_{22}^{\prime \prime}\right) .
\end{aligned}
$$

We need to say a word about how we check Equation 117 in the context of interval arithmetic computations. When we write $x=0$ we actually mean that the interval representing $x$ has endpoints with opposite signs. This is to say that $x$ is indistinguishable from 0 . When we say $x>0$ we mean that both endpoints of the interval representing $x$ are positive. And so on.

If $b_{0}^{2}-4 a_{0} c_{0}<0$ then the roots of $P$ are imaginary and Equation 117 has no solutions. If $a_{0} b_{0}>0$ and $a_{0} c_{0}>0$ then any real roots of $P$ are negative, so again Equation 117 has no solutions. If these two tests fail, we try more specialized tests.

If $a_{0}>0$ then Equation 117 is equivalent to

$$
\begin{aligned}
& P(t)=0 ; \quad t>0 ; \quad a_{2} t+b_{2}>0 ; \\
& a_{2}=a_{0} b_{1}-a_{1} b_{0} ; \quad b_{2}=a_{0} c_{1}-a_{1} c_{0} .
\end{aligned}
$$


If either $a_{2}=0$ or $b_{2}=0$ we fail the computation. This leaves 4 cases, depending on the signs of $a_{2}$ and $b_{2}$. For instance, if $a_{2}<0$ and $b_{2}>0$ then $t$ must lie in $(0, x)$, where $x=-b_{2} / a_{2}$. From here, the number of solutions can be determined from the signs of $P(0), P(x), y, y-x$, and $P(y)$. (Here $y=-b_{0} / 2 a_{0}$ is the critical point of $P$.) If $P(0)$ and $P(x)$ have opposite signs then Equation 118 has one solution. If $P(0)$ and $P(x)$ have the same sign and $x_{0} \notin[0, x]$ then Equation 118 has no solutions. And so on.

If $a_{0}<0$ the computation is similar, except that $a_{2}$ and $b_{2}$ change sign.

If $a_{0}=0$ and $c_{0} \neq 0$ then we parametrize $S^{\prime \prime}$ by $S_{1}^{\prime \prime}+t S_{2}^{\prime \prime}$ instead. This leads to a variant of Equation 117 in which $a_{j}$ and $c_{j}$ are switched for $j=0,1$.

If $a_{0}=c_{0}=0$ we try a completely different method. We compute the midpoint $m$ of the segment joining the two endpoints of $\left[S^{\prime \prime}\right]$, and consider the disk $D$ centered at $m$ and containing these two endpoints. The point $p=\left[S_{1}^{\prime \prime} / 2+S_{2}^{\prime \prime} / 2\right]$ is an interior point of $\left[S^{\prime \prime}\right]$. If $p \in D$ then $\left[S^{\prime \prime}\right] \subset D$. In this case we try to show that the $x$-coordinate of $m$ is more negative than the radius of $D$ is positive. This shows that $D$, and hence $\left[S^{\prime \prime}\right]$, is disjoint from $[0, \infty]$.

We fail the computation in all circumstances not already covered.

\subsection{Record of the Calculatons}

I ran all the computer experiments for this paper on my home computer, which is a Sparc Ultra 10. The operating system for the computer is Solaris 7. I used the GNU C compiler, and compiled the code without any optimization flags. I ran the calculations, successfully, on 15 January 2002. To give some statistics, the computer tested 1362633 pairs of tetrahedra which had 0 vertices in common, 21117 tetrahedra which had 1 vertex in common, 4440 tetrahedra which had 2 vertices in common, and 1458 tetrahedra which had 3 vertices in common. 


\section{List of Important Symbols}

\begin{tabular}{|c|c|c|c|}
\hline symbol & definition & 1st mention & lookup \\
\hline$Z, Q, R, C$ & integers, rationals, reals, complexes & & \\
\hline $\boldsymbol{H}^{n}$ & real hyperbolic $n$-space & $\S 1.0$ & \\
\hline $\boldsymbol{C} \boldsymbol{H}^{n}$ & complex hyperbolic $n$-space & $\S 1.0$ & $\S 2$ \\
\hline$Q \boldsymbol{H}^{n}$ & quaternionic hyperbolic $n$-space & $\S 1.0$ & \\
\hline$P U(2,1)$ & projective unitary group & $\S 1.0$ & $\S 2$ \\
\hline$S^{3}$ & unit 3-sphere & $\S 1.0$ & \\
\hline$G(4, n)$ & basic abstract group & $\S 1.1$ & $\S 1.1$ \\
\hline$\rho(4, n)$ & the basic representation & $\S 1.1$ & $\S 3.1$ \\
\hline$\Gamma(4, n)$ & image of $G(4, n)$ under $\rho(4, n)$ & $\S 1.1$ & $\S 3.1$ \\
\hline$I_{1}, I_{2}, I_{3}$ & generators of $\Gamma(4, n)$ & $\S 1.1$ & $\S 3.1$ \\
\hline$\Lambda(4, n)$ & limit set of $\Gamma(4, n)$ & $\S 1.1$ & \\
\hline$\Omega(4, n)$ & domain of discontinuity of $\Gamma(4, n)$ & $\S 1.1$ & \\
\hline$Z$ & the basic simplicial complex & $\S 1.1$ & $\S 4$ \\
\hline$Z^{\prime}$ & refinement of $Z$ & $\S 1.1$ & $\S 9$ \\
\hline [ ] & projectivization map & $\S 1.3$ & $\S 2$ \\
\hline$[Z],\left[Z^{\prime}\right]$, etc. & projective images of $Z, Z^{\prime}$ etc. & $\S 1.3$ & \\
\hline$\left[Z_{0}\right]$ & intersection of $[Z]$ with $S^{3}$ & $\S 1.3$ & \\
\hline$\left[Z_{0}^{\prime}\right]$ & intersection of $\left[Z^{\prime}\right]$ with $S^{3}$ & $\S 1.3$ & \\
\hline$\Delta_{0}$ & component of $S^{3}-\left[Z_{0}\right]$. & $\S 1.3$ & \\
\hline$Z_{-}$ & negative part of $Z$ & $\S 1.3$ & \\
\hline$\Delta_{-}$ & component of $\boldsymbol{C} \boldsymbol{H}^{2}-\left[Z_{-}\right]$ & $\S 1.3$ & $\S 8$ \\
\hline$E_{*} K$ & eigenvectors of $K$ & $\S 1.3$ & $\S 4.1$ \\
\hline$Z_{0}^{*} K_{*}$, etc. & real hyp. analogs of $Z_{0}, K$, etc. & $\S 1.3$ & $\S 5$ \\
\hline$h$ & homo from $\Gamma^{*}(4,7)$ to $\Gamma(4,7)$ & $\S 5.4$ & $\S 5.4$ \\
\hline$h^{\prime}$ & homeo from $\Delta^{*}$ to $\Delta$ & $\S 1.3$ & $\S 5.4$ \\
\hline$h^{\prime \prime}$ & covering map of $h^{\prime}$ & $\S 5.4$ & $\S 5.4$ \\
\hline$\langle\rangle$, & Hermitian inner product & $\S 2$ & $\S 2$ \\
\hline$N_{-}, N_{0}, N_{+}$ & neg., null, pos. vectors & $\S 2$ & $\S 2$ \\
\hline$\varrho$ & complex hyperbolic metric & $\S 2$ & $\S 2$ \\
\hline$\delta$ & projective invariant related to $\varrho$ & $\S 2$ & $\S 2$ \\
\hline$S U(2,1)$ & $\langle$,$\rangle -preserving group$ & $\S 2$ & $\S 2$ \\
\hline 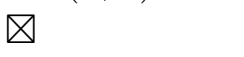 & Hermitian cross product & $\S 2$ & $\S 2$ \\
\hline & complex reflection fixing vector $C$ & $\S 2$ & $\S 2$ \\
\hline$V_{1}, V_{2}, V_{3}$ & vectors defining $\Gamma(4,7)$ & $\S 3.1$ & $\S 3.1$ \\
\hline$C_{1}, C_{2}, C_{3}$ & auxilliary vectors for $\Gamma(4,7)$ & $\S 3.1$ & $\S 3.1$ \\
\hline
\end{tabular}




\begin{tabular}{|c|c|c|c|}
\hline symbol & definition & 1st mention & lookup \\
\hline$\kappa_{1}, \kappa_{2}, r, s, t$ & variables for the $V_{j}$ & $\S 3.1$ & $\S 3.1-3.2$ \\
\hline$J, J^{\prime}, K$ & auxilliary elements of $S U(2,1)$ & $\S 3.2$ & $\S 3.2$ \\
\hline$\Upsilon$ & network of real slices & $\S 3.3$ & $\S 3.3$ \\
\hline$\Upsilon^{\infty}$ & ideal boundary of $\Upsilon$ & $\S 3.3$ & $\S 3.3$ \\
\hline$R$ & the trace number ring & $\S 3.4$ & $\S 3.4$ \\
\hline$A(m), B(m)$ & pieces of $Z$ & $\S 4.1$ & $\S 4$ \\
\hline$A_{m}, B_{m}$ & pairing reflections of $A(m), B(m)$ & $\S 4.9$ & $\S 4.9$ \\
\hline & coordinate chart used for plotting & $\S 4.1$ & $\S 4.1$ \\
\hline$[12]$, etc. & word notation for fixed points & $\S 4.2$ & $\S 4.2$ \\
\hline$\bullet j, \circ j$ & chart notation for fixed points & $\S 4.2$ & $\S 4.2$ \\
\hline$\bowtie$ & join operator & $\S 4.4$ & $\S 4.4$ \\
\hline$\lambda, a_{0}, b_{0}, a_{1}$ & variables in the def'n of $\Gamma^{*}(4,7)$ & $\S 5.2$ & $\S 5.2$ \\
\hline$\alpha_{n}, \beta_{n}$ & axes for pairing halfturns & $\S 5.3$ & $\S 5.3$ \\
\hline$\alpha_{n}^{0} \beta_{n}^{0}$ & centers of symmetry on halfturn axes & $\S 5.3$ & $\S 5.3$ \\
\hline$\alpha_{n}^{+}, \beta_{n}^{-}$, etc & tiling vertices & $\S 5.3$ & $\S 5.3$ \\
\hline$Q_{0}^{*}, Q_{01}^{*}, Q_{02}^{*}$ & pieces used in defining orbifold & $\S 5.5$ & $\S 5.5$ \\
\hline$Z_{1}, Z_{2}, Z_{3}$ & layers of $Z$ & $\S 6.1$ & $\S 6.1$ \\
\hline$A(n, \pm)$ & parts of piece $A(n)$ & $\S 6.1$ & $\S 6.1$ \\
\hline$T_{+}, T_{-}$ & pos and neg parts of tetrahedron $T$ & $\S 6.2$ & $\S 6.2$ \\
\hline & quotient of $\Delta_{-} \cup\left[Z_{-}\right]$by $K^{3}$ & $\S 7.1$ & $\S 7.1$ \\
\hline$O, O_{1}, O_{2}$ & spaces associated to $Q_{-} / \sim$ & $\S 7.2$ & $\S 8.2$ \\
\hline$R_{P}$ & reflection pairing of piece $P$ & $\S 7.2$ & $\S 7.2$ \\
\hline$\#$ & conservative barycentric subdivision & $\S 8.2$ & $\S 8.2$ \\
\hline$\mu, \mu_{j}$ & perturbation data for $Z^{\prime}$ & $\S 8.3$ & $\S 8.3$ \\
\hline$T_{1}, \ldots, T_{n}$ & basic list of tetrahedra & $\S 9.1$ & $\S 9.1$ \\
\hline$T^{(m)}$ & $m$ th subdivision of $T$ & $\S 9.1$ & $\S 9.1$ \\
\hline$(i, j, k)$, etc. & code for vertices & $\S 9.2$ & $\S 9.2$ \\
\hline$\widetilde{Z}^{\prime}$ & combinatorial version of $Z^{\prime}$ & $\S 9.2$ & $\S 9.2$ \\
\hline$C \widetilde{Z}^{\prime}$ & computational part of $\widetilde{Z}^{\prime}$ & $\S 9.3$ & $\S 9.3$ \\
\hline$\Pi_{j}, \Pi_{K}, \pi_{K}$ & projections related to $K$ & $\S 9.3$ & $\S 9.3$ \\
\hline$T \# T^{\prime}$ & number of common vertices & $\S 9.4$ & $\S 9.4$ \\
\hline$T \square T^{\prime}$ & convex hull of $T \# T^{\prime}$ & $\S 9.4$ & $\S 9.4$ \\
\hline$T \searrow T^{\prime}$ & $T$ minus $T \square T^{\prime}$ & $\S 9.4$ & $\S 9.4$ \\
\hline$z(p, q, n)$ & pseudorandom complex number & $\S 9.6$ & $\S 9.6$ \\
\hline $\operatorname{Proj}_{n}$ & $n$th pseudorandom projection & $\S 9.6$ & $\S 9.6$ \\
\hline$\langle U\rangle$ & set based on 1-skeleton of $[U]$. & $\S 9.6$ & $\S 9.6$ \\
\hline$T_{e}$ & wedge of $e$ and $T$ & $\S 9.8$ & $\S 9.8$ \\
\hline
\end{tabular}




\section{References}

[A] R.D. Anderson, A Characterization of the Universal Curve and a Proof of its Homogeneity, Annals of Math 67 (1958)

[B], A.F. Beardon, The Geometry of Discrete Groups, Graduate Texts in Mathematics 91 (1983) Springer-Verlag

[BLP $]$ M. Boileau, B. Leeb, and J. Porti, Uniformization of Small 3-Orbifolds, preprint (2001)

[C] K. Corlette, Archimedean Superrigidity and Hyperbolic Geometry, Annals of Math. 135 (1992) 165-182

[CHK] D. Cooper, C. Hodgson, and S. Kerkhoff, Three Dimensional Orbifolds and Cone Manifolds, Proceedings of the Mathematical Society of Japan $5(2000)$

[DM $]$ P. Deligne and G.D. Mostow, Commensurabilities among Lattices in PU $(1, n)$, Annals of Mathematics Studies 132, Princeton University Press (1993)

[E] D.B.A. Epstein, Complex Hyperbolic Geometry, London Mathematical Society Lecture Notes, 111 (1987)

[FH] W. Floyd and A. Hatcher, Incompressible Surfaces in Punctured Torus Bundles, Topology and its Applications 13 (1982) 263-282

[FZ $]$ E. Falbel and V. Zocca, A Poincare's Fundamental Polyhedron Theorem for Complex Hyperbolic Manifolds, J. reine agnew Math. 516 (1999) 133-158

[G] W. Goldman, Complex Hyperbolic Geometry, Oxford Mathematical Monographs, Oxford University Press (1999)

[GKL] W. Goldman, M. Kapovich and B. Leeb, Complex Hyperbolic Surfaces Homotopy Equivalent to a Riemann surface, Communications in Analysis and Geometry 9 (2001) 61-95 
[GP] W. Goldman and J.R. Parker, Complex Hyperbolic Ideal Triangle Groups, J. reine agnew Math. 425 (1992) 71-86

[GrP] M. Gromov and I. Piatetski-Shapiro, Nonarithmetic Groups in Lobachevski Space, Publ. I.H.E.S. 66 (1988) 93-103

[GrS] M. Gromov and R. Schoen, Harmonic Maps into Buildings and padic Superrigidity for Lattices in Groups of Rank One, Publ. I.H.E.S. 165 (1992) 165-246

[GuP $]$ N. Gusevskii and J.R. Parker, Representations of Free Fuchsian Groups in Complex Hyperbolic Space, Topology 39 (2000) 33-60

[I] Institute of Electrical and Electronic Engineers, IEEE Standard for Binary Floating-Point Arithmetic, July 26, 1985

[K] N. Koblitz, Introduction to Elliptic Curves and Modular Forms, Graduate Texts in Mathematics, Springer Verlag 97 (1984)

[KR] B.W. Kernighan and D.M. Ritchie, The C Programming Language, Prentice Hall Software Series (1978)

[KK] M. Kapovich and B. Kleiner, Hyperbolic Groups with Low Dimensional Boundary, preprint

[KT] Y. Kamishima and T. Tsuboi, CR Structures on Seifert Manifolds, Invent. Math. 104 (1991) 149-163

[MV] A. Mednykh and A. Vesnin, On the Fibonacci Groups, the Turk's hear links, and Hyperbolic 3-Manifolds, Groups-Korea (1994) 231-239

[MW] J. Montesinos and W. Whitten, Construction of 2-fold Branched Covering Spaces, Pacific J. Math. 125 (1986) 415-446

[O] J. K. Ousterhout, Tcl and the Tk Toolkit, Addison-Wesley (1994)

[S1] R.E. Schwartz, Ideal Triangle Groups, Dented Tori, and Numerical Analysis, Annals of Math 153 (2001) 
[S2] R.E. Schwartz, Degenerating the Complex Hyperbolic Ideal Triangle Groups, Acta Mathematica 186 (2001)

[S3] http://www.math.umd.edu/ res

[Sh] Y. Shalom, Rigidity, Unitary Representations of Semisimple Groups, and Fundamental Groups of Manifolds with Rank One Transformation Group, Annals of Math 152 (2000) 113-182

[Sp] E. Spanier, Algebraic Topology, Springer-Verlag, 1966

[T1] W. Thurston, Three Dimensional Geometry and Topology, Princeton Mathematical Series (1998)

[T2] W. Thurston, Shapes of Polyhedra and Triangulations of the Sphere, Geometry and Topology Monographs 1, ed. I Rivin, C. Rourke, C. Series, The Epstein birthday shrift (1998) 511-549 (electronic)

[Tol] D. Toledo, Representations of Surface Groups on Complex Hyperbolic Space, Journal of Differential Geometry 29 (1989) 125-133

[W] S. Wolfram, The Mathematica Book, Fourth Edition, Cambridge University Press (1999)

[W-G] J. Wyss-Gallifent, Discreteness and Indiscreteness Results for Complex Hyperbolic Triangle Groups, Ph.D. Thesis, University of Maryland (2000) 